\title{
U.5. miviler
}

\section{Tile-law c, op \\ iralares}

57.8

$m 885$

20

$\infty$

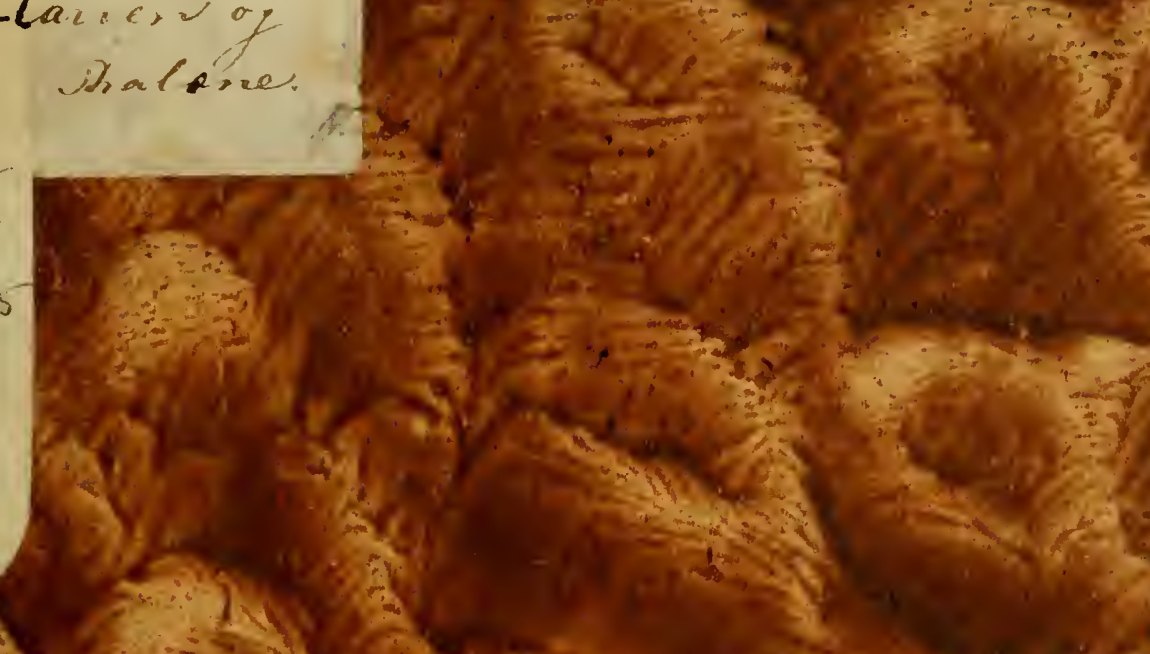

$\frac{d}{4}$

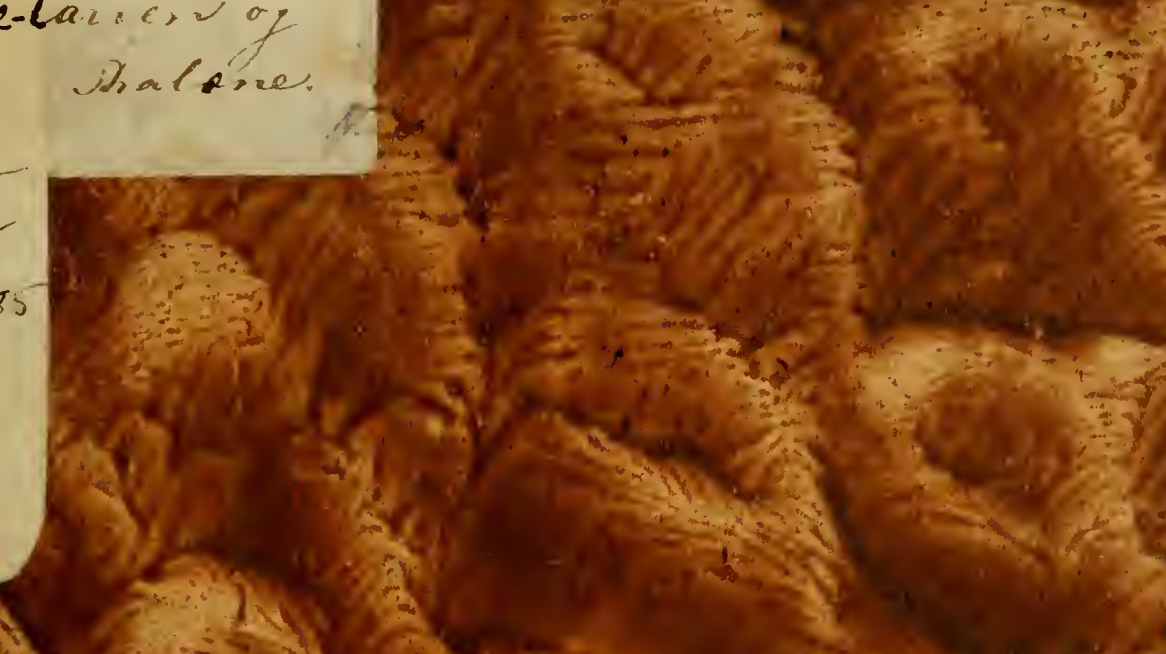

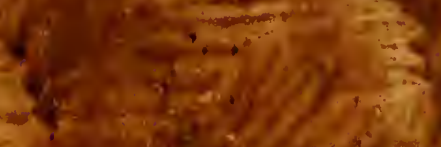

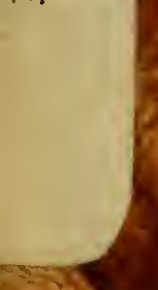

(a)

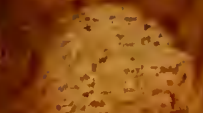

25
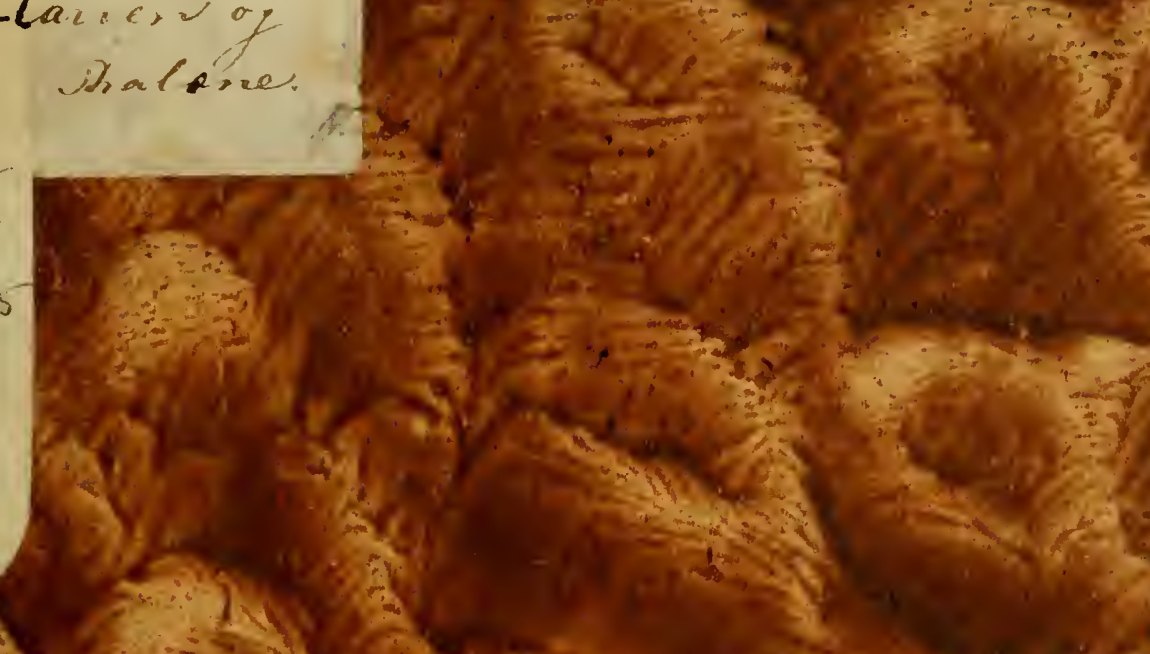

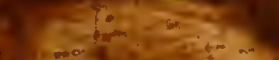

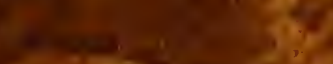

$-4$

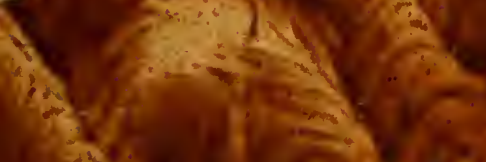

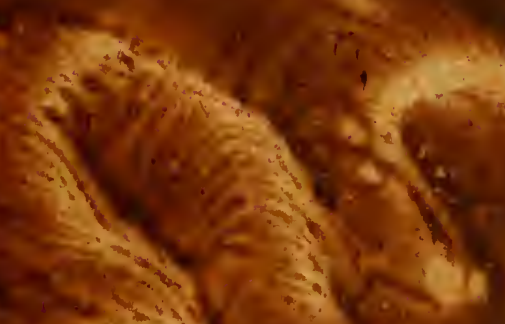

21

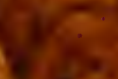

8
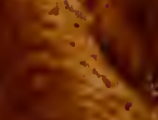

tin.

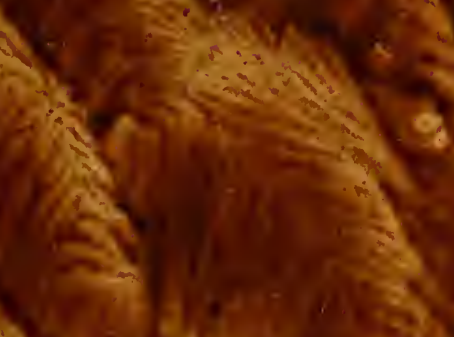

$\sin$

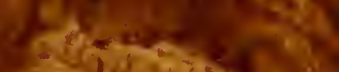

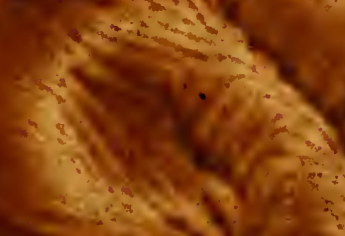

$x^{2}+3$

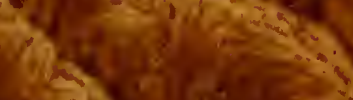

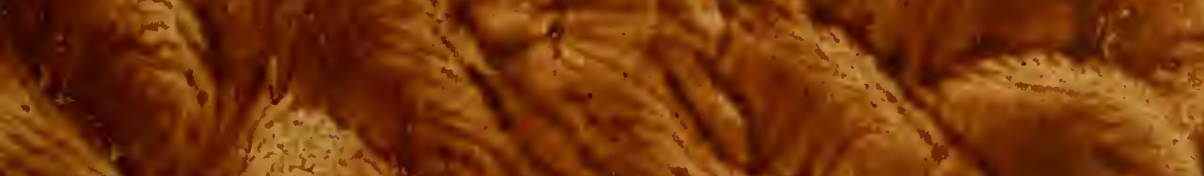

$3 x=$

40

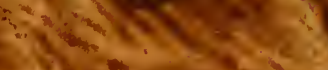

$x y^{4}=$.

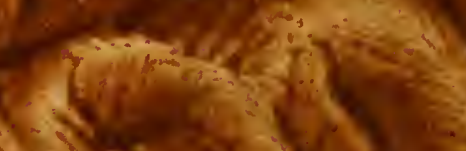

(4)

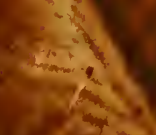

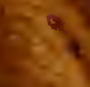

$\therefore \div$

8

8

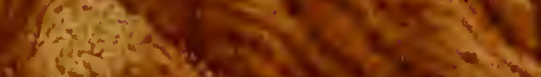

3.1.

$\lim _{\infty} \lim _{-\infty}$

-

$p^{2}$

3

1) $1 y^{2}=6$

f.

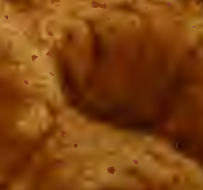

38

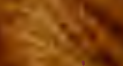

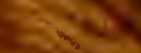

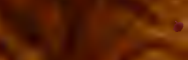

to.

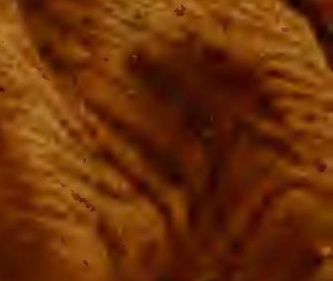




\section{$7 \cdot 26.07$}

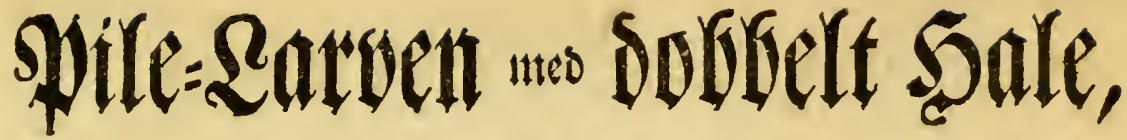

og Detlô

\section{$\mathfrak{W h} \mathfrak{a} \mathfrak{L} \mathfrak{a} \mathfrak{n} \mathfrak{e} ;$}

mes

Gozfonede Bemarefelfer,

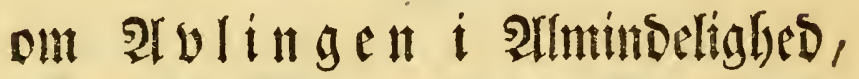

og Syampenes siblibelie,

\section{af \\ Dtto Friderich Muller}

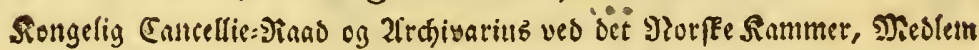

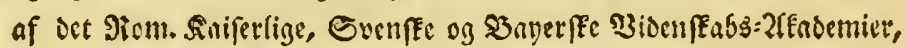

fom og af bet S?orfere og andere Gelfeaber.

\section{Noi fram vermi}

Nati a formar l'angelica Farfalla. D.NNe.

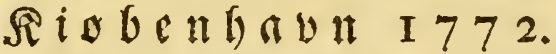

Tryet paa det Typographife Selfeabs 3efoltutilg, ḩos Motten Scallager, boetise fan Dietregabe i Dio. 245.

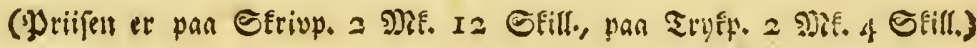




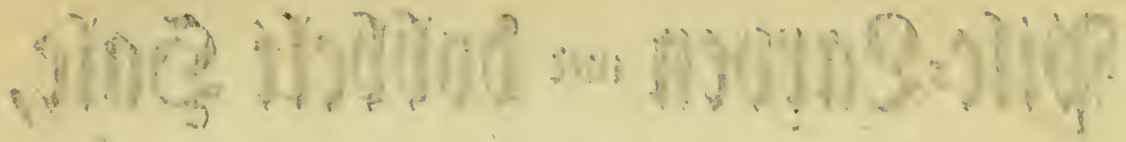

$$
\begin{aligned}
& \therefore 14,2 \times 1
\end{aligned}
$$

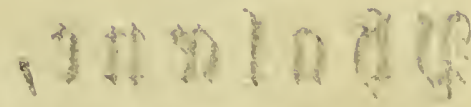

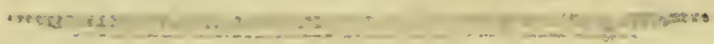

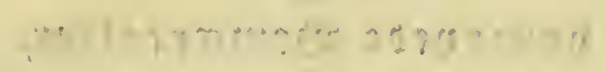

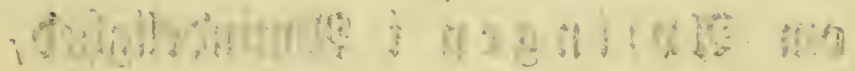

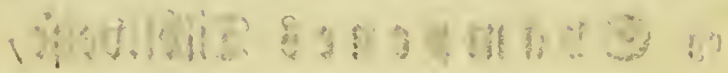

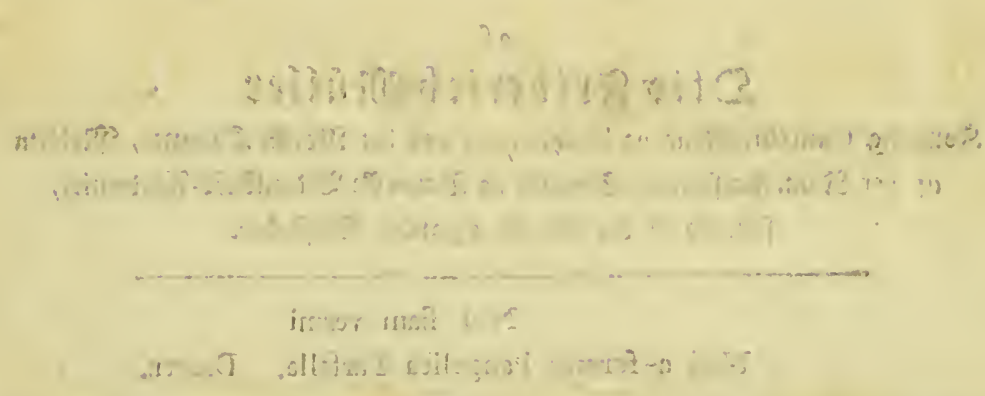

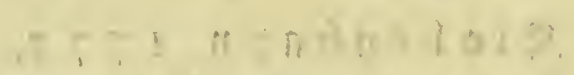

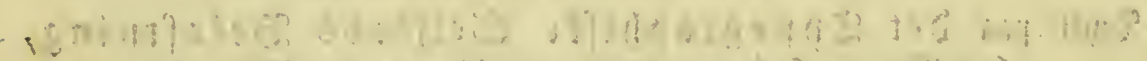

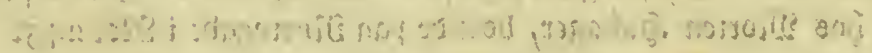

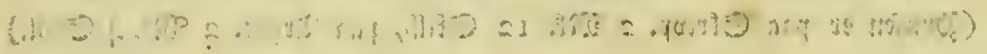


Iil

Sanb songelige Saybed

Prittos Ftidetid

- 2 


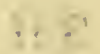

$$
\begin{aligned}
& \text { b. }
\end{aligned}
$$

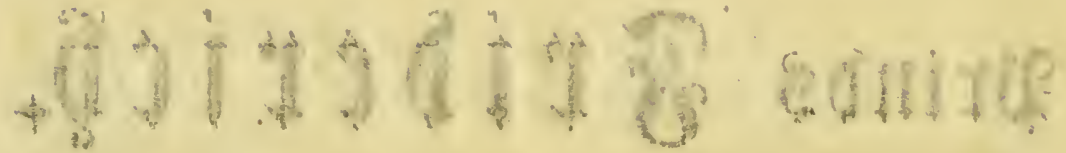




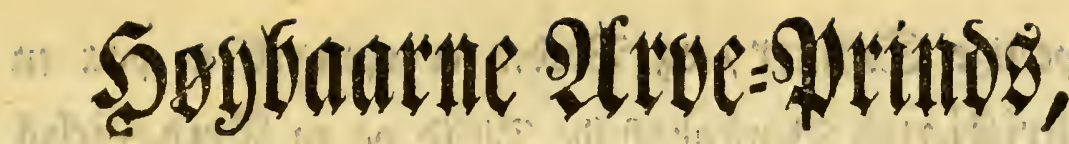

\section{Padadigfte $\mathfrak{s e r t e , ~}$}

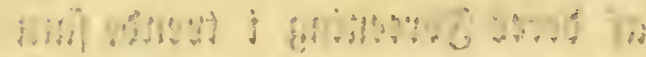

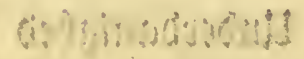

puntuntuet af Den nandige $23 e l b e f a g$, Mted buifket

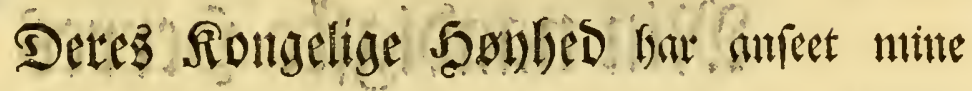

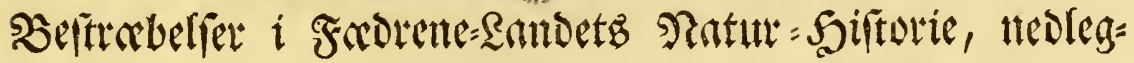
ger jeg untoctomigft dette Serift for en Forites some,

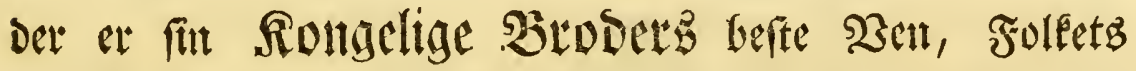
Talsintmo og Thronens Stotte; and mere, fom med lige Stiffed, ag itted lige Bifallo fat levede $i$ fin ontes

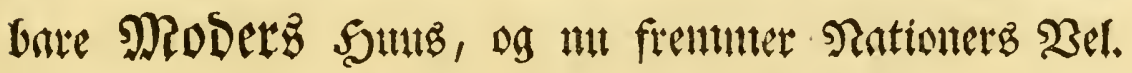
Suat Nordenz Satder fordun fang oun Detes Ron= gelige 5 and bed 
vppertige Sonner, og Berden et oputretefom paa en

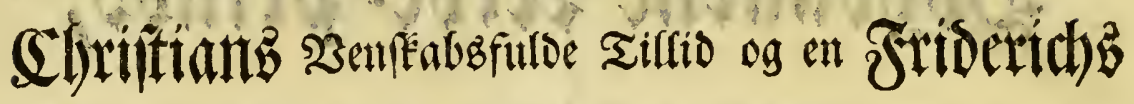
gmme Sengivented. Sleve diffe Naune, fuert for fig,

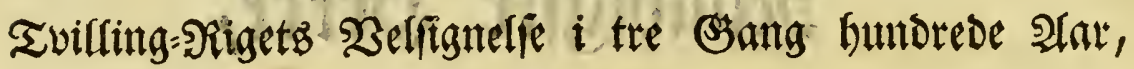
lowo tar bet on vente af beres foreening $i$ twende fam= orretige $\mathfrak{B r o b r e !}$

Seg forbliver $i$ dubefte Underdonighed

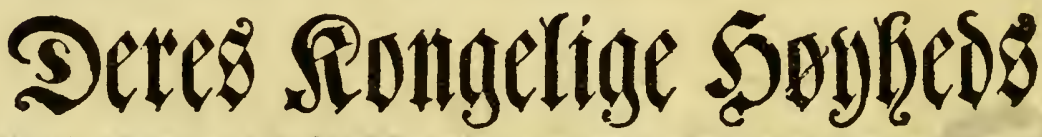 Min nadadgfte $\mathfrak{s e t r t \mathfrak { B }}$}

Rishenliun, den 20 2fugufs

1772.

insoctoanigfte

Dtto Friberich, Mutter. 


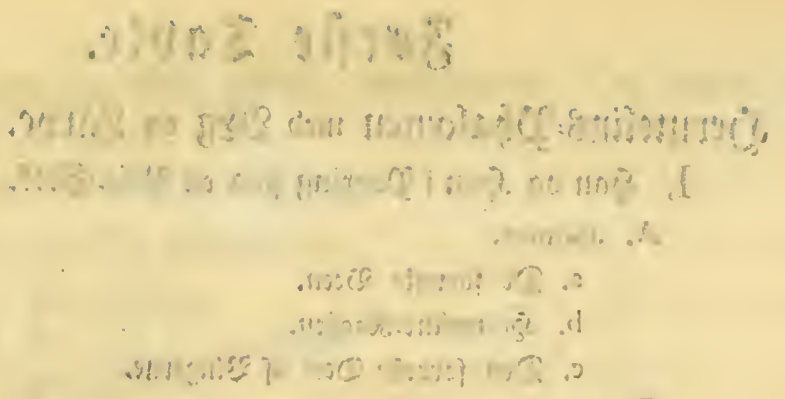

$\Omega$

$\therefore:-410 \% \pi$

Uyb par N.xturens Ttoft! en Orm, et Grant sig Guber:

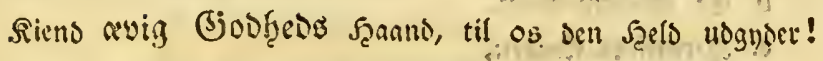

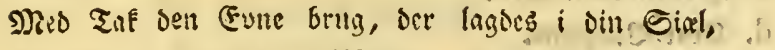

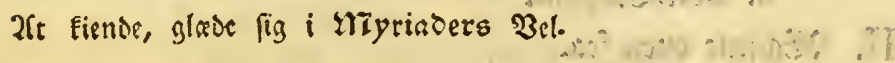

Snlg i phalenents Spor: oen fext til Minalet iler.

23ids! Du ved fiofte Trin fra Gavte slane Griler;

Frygt en for Tidens \&ce, fuft glad Din Larne ben -

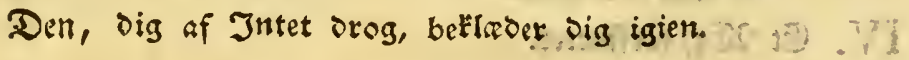
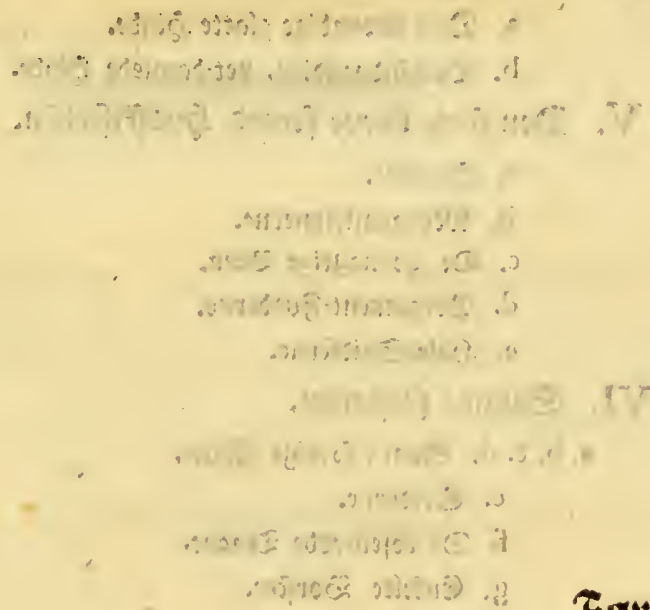


\section{Sorfte $\mathfrak{T a b l e}$.}

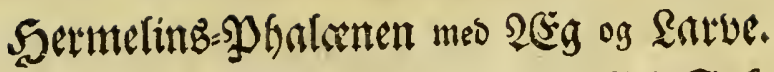

I. Scan og Şun $i$ Parring paa en $\mathfrak{\text { Pile }}=$ Stol.

A. Şannen.

a. De forrefte Deen.

b. Şermelinz:-Rnaben.

c. Den fortefte Deel of গYingerne;

B. Şนuัner.

a. De fortefte Siectr.

b. Et Viellem=Been.

c. Scermelinb: Snafict.

d. Dver=2zingerne.

II. 2ergene oven fra:

III. 2Eggene nebén frá.

a. Den optrevede Finto.

b. Den Elare Scinde.

IV. Ett 2Eig, forfterter.

a. Den ubvendige glatte Seinde.

b. Den insuendige, net:dannede Şinde.

V. Den forte Earve fareno Shud Eiftelen.

a. Szovedet.

b. Norgenftiernerne.

c. De bormatige Deen.

d. Pergament=Fobserne.

e. Şale:Spioferne.

VI. Samme forfterret.

a. b. c. d. Som i forrige Sigut.

e. Sfeberne.

f. De rofen=rode Traade.

g. Eneflte Sorfter. 
VII. Earven ofter forfte Sthelfe, fiarmefin reo, i en opbragt Etil= ning.

VIII. Sarven efter anton Şutberfe.

IX. Earven, nepten fulbsoren.

a. \ante:yliden.

b. Den rede og forte Seinde.

c. Jole: Scornente.

d. De porte Sçuller.

e. Dell rabe Snop.

f. De Gornagtige $\mathfrak{B e n}$.

g. \ergament:

h. Elfft:Scullerne.

i. Spusene, eller be fpillende Trante.

k. Sefoerne.

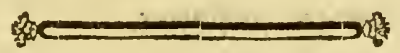




\section{ҮIII}

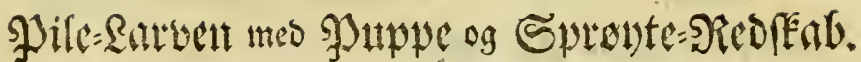

I. Rarven mes bobrelt Sale, fuloworen.
a. Pambeyladen.
h. Ting:Sitoppen.

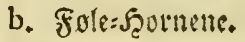
i. Enft:Şullerne.
c. 20:0e:Spibjerne.
k. De Gorragtige Sicelt.
d, Din forte Şinde.
1. Dergament: Fodoctre.
e. Din rofentrabe Sinde. m. Dent tode Side:z)iet.
f. จำ
n. Eprsenc. -
g. Di forte Sentlet.
o. Sfcuert.

p. Tnende forte §ortfret.

II. Enruen par Etitem of at aforot Wite-Bjab.

III. Earve Şoveset, libet feerte end naturligt.
a. Den rod STitroc.
c. De porte Sezuller.
b. Din forte șinte.
d. Tuct:-Epraten, fyorfir Bzandet ubiprentes.

IV. Sprente=Picopleabet mes fine fire Epitere, forfteret.

V. En Spidfe, meget forftorret.

a. En med Şuller befat 3orte.

b. Dit parpe SEaft.

VI. ENn $\mathfrak{b} n g=\mathfrak{F} n$, forfterret.
a. 2319 : Revet.
c. Den potte Siant mes Sortfer.
b. Didfem:Eedet.
d. Foopanten med fine \$ortfter.

VII. Soffer, faftipmet sg eltinet paa et Stylepe Tre. VIII. Spuppen, foun Den fees inden for Solfen.

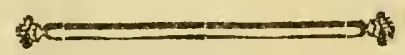




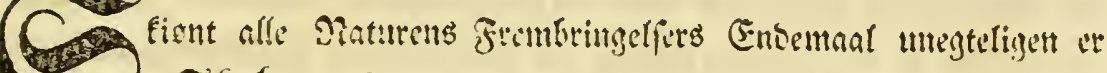

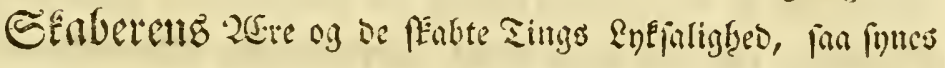
oog nogle at have neycre beftemte Dyemco, og alle at fraat

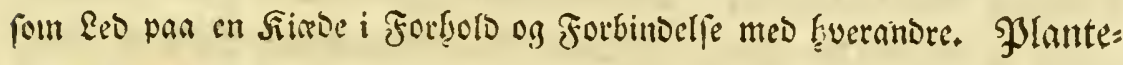
Siget Ean lige fan liset untuare Dyre=-Piiget, font Dyrente Mlanterne, og besge vare iffe til uben Sten = Sitget; og fyad vare alle tre uben se

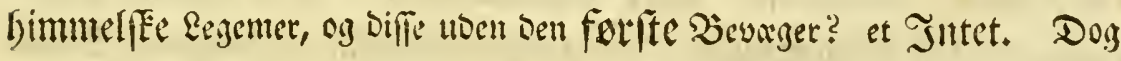
finbe si, biffe almindelige forbindelfer ungtet, at en Slabning nermert anganet Den anben ento Den trebic. Del Infer Suclen for alle Dnr, og

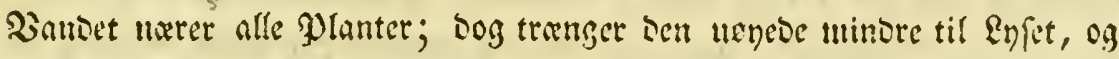

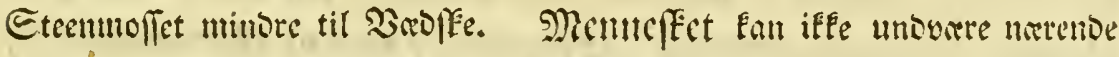

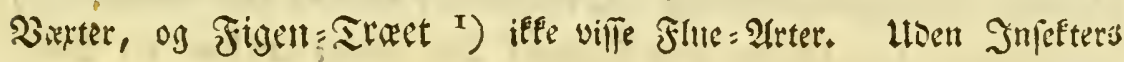
For $=$

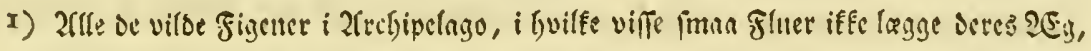

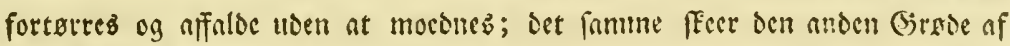

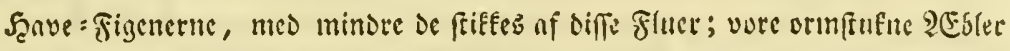
og Jyarer blive og frarere moestre, end de, fom iffecte det. Det viloe vare fordeclangtigt, Gvis den Girablfece Caprification funde anbringes para vore 2fpris cofer, fom fia fielden mocolles. 
Forraabuele vorber ingen Safrutb: Solle ${ }^{2}$ ) og uben Whanters Splesning intet Infuftons=Dyk. Mange Flue=?rter vilbe forgane, f̧vis ingen Earver gaves, og Hoen Menmefert vilde viffe Orme opḩere.

Sa anfer vi bem allene fom Bientano for vore befferftigelfer, Da fiar

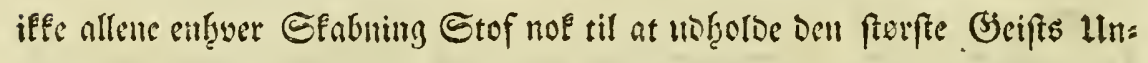

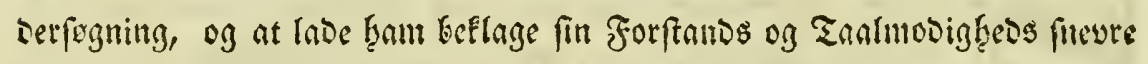

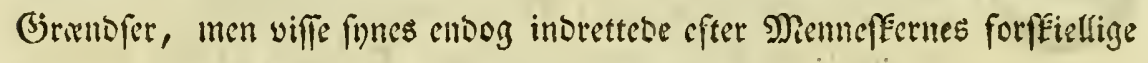

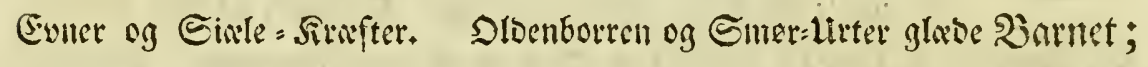

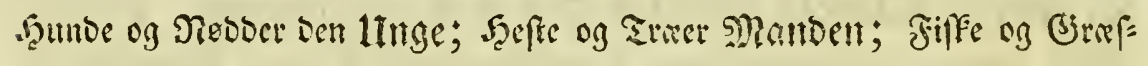
fet ben Giamle. Furfterme behage fig i fremmede Barters Dunfer, og

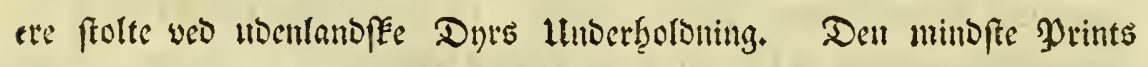

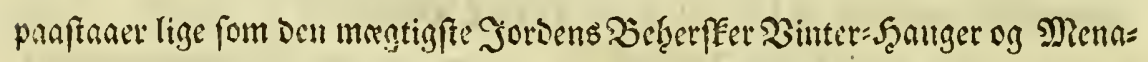
gerier; et Mlommerants= Tre og en Maafugl frobe begge $i$ beftandig 3 an= fundighed on Deres egne Rantes naturlige Enendoumme; fivilfen Songe fyar, efter Salomon, nivet fine Rigers Natur = \$prooutfter 2rogatg til

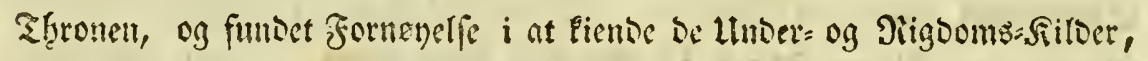

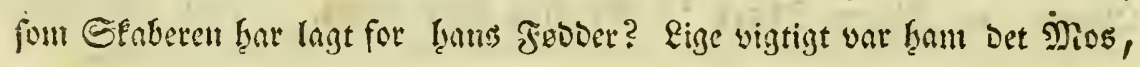

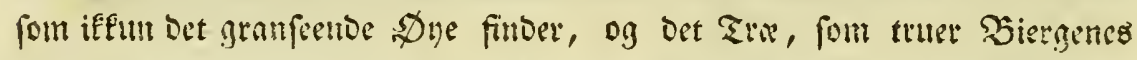

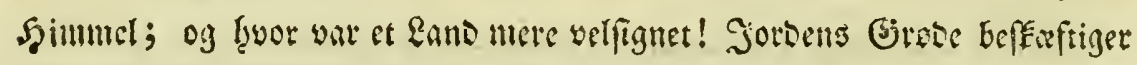

LAID:

2) Clavaria militaris, of Franfer mouche vegetante, fon iffe fromfonmer usturaa

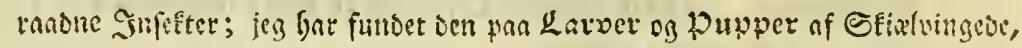
sg paa de Dinge:DafFeoc (coleoptern) og beffrevet den i Nov. net, nat, curiof. tom. 4, obf. $45, t, 7, f, 5, G$. 


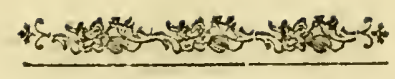

3

Eanbmansan og f̧ayets Formad Etrand = Stbberen; Bromfer og

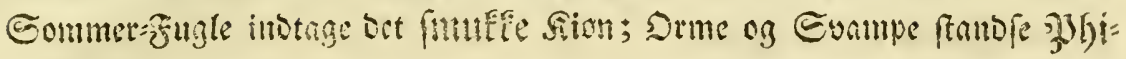

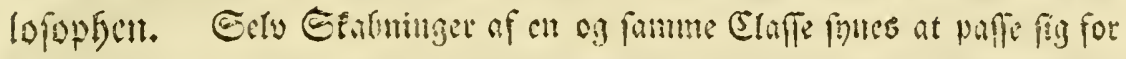

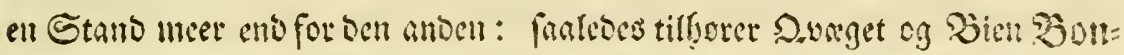

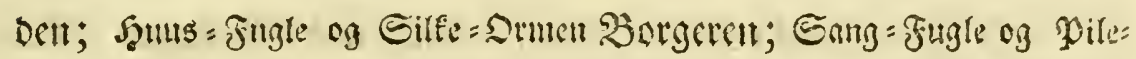
Earven ben mugeligere.

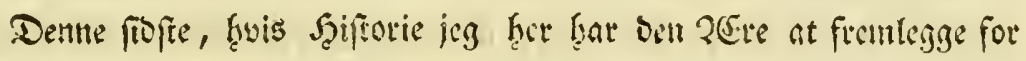

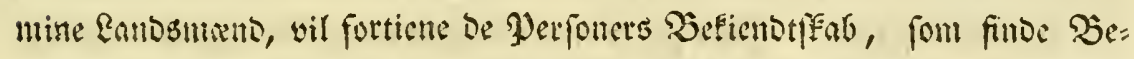
frans $i$ Siaberens Birrninger, og de Smuffes Dumgang fram for mange

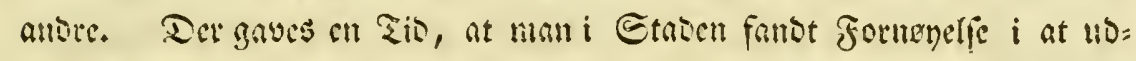
flaffe Giffes Dome, mere for at tilfee Deres Forvandinger, eno for at

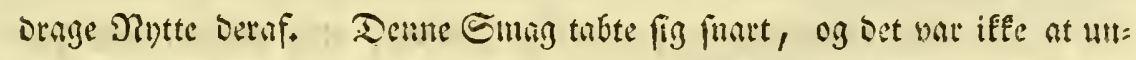
bre; Gilfes:Drmen er iffe fentat at rores af wore Efiantevers fingre, eller

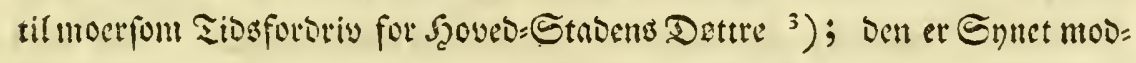

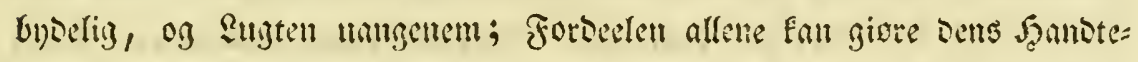

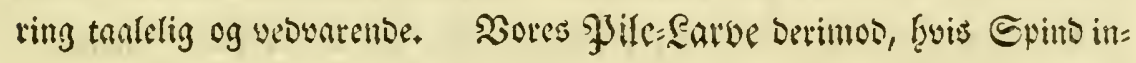
I 2 tet

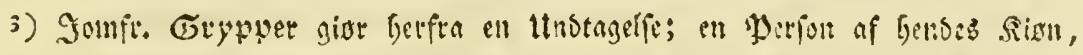

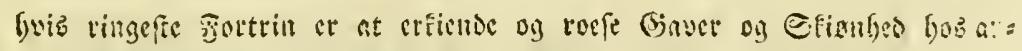

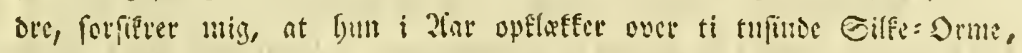

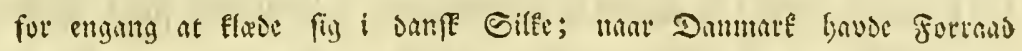

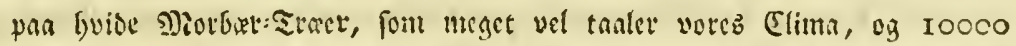

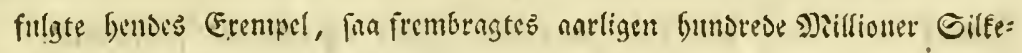

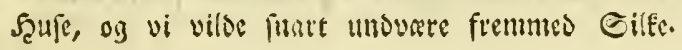


tet Formaal giver for Binditn og Şandel, f̧ar bet, fom vil beţage ben Spmerefomme og oen Fitte.

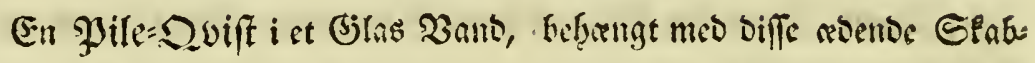
nitrger af forffiellig $\mathfrak{A l d}$ er: Deres Eegenters foranderlige Etilling og Far=

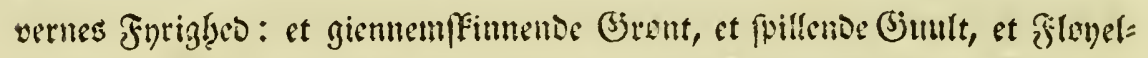
lignende Sort, et levende Root, og et matbleven Soviot giver et Malerie, fom henrnfler \$yei. Man fee Desuben et trittot og frot Regeme i Baglige=

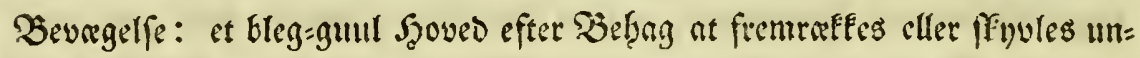

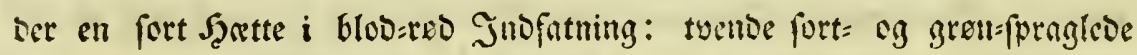
Sfeser opreift fra Şalen, og of siffe til Srmens Forfuar at ubfare lige= faa mange lange rofen=robe og beyergelige Trande, eller mellem bens for=

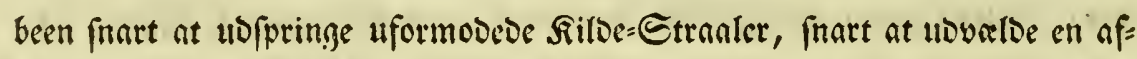

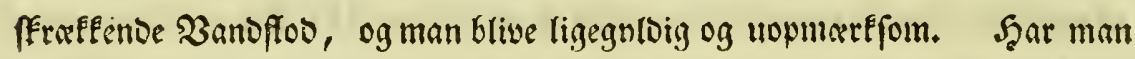
Suervindelfe nof til at give Dem nogle \$oyeblifs $\mathfrak{P l e n e}$ fever anten Dong, at tillee Deres Şutstroloning og Forvanolinger, fan wil be fuart intereffere baade Şiertet og Forftanden.

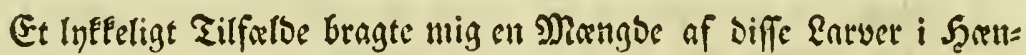

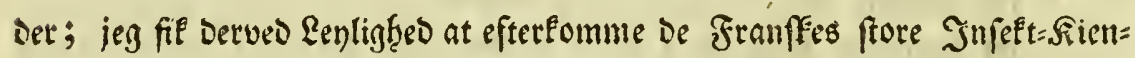
Dere Şr. Fienumirrz Forlangende, ved at unberfoge Denne merfvarbige $\mathfrak{T}$ trt, f̧voraf f̧an troct, at iflun have feet twende og allerede fulbyopne. Scans Figut= 
Figurer ${ }^{4}$ ) vife, at fran fraver feet fece i beris ferffiellige strot, men iff: fiendt, at be vare en og ben panme 2 trt.

Den I4 May I760 var jeg uDgangen efter Sabvane i Morgenfun=

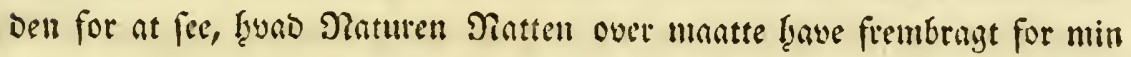
Ninfigenthes, Glant andet falot bet mig itto at efterfee nogle Yiles:etoffe, foul jeg for tyende llger havade neb fat til Frement. Sicppe bavod jeg

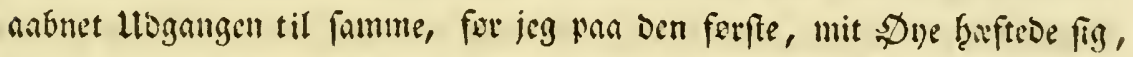
bley yaer neget graat og laadent. Shaly Frngt for et ubefientot Dont,

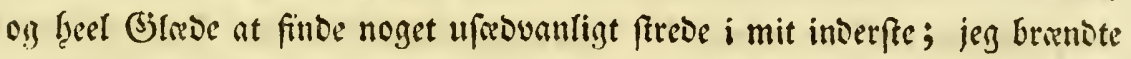

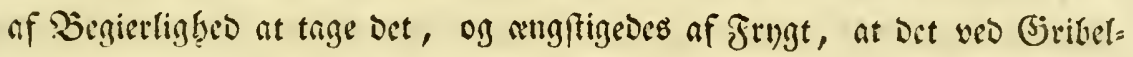

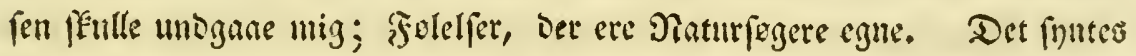
A 3

mig

4) Memoires fur les infectes, tom. 2. part. 2. planche 2 I \& 22. f. I. 2.3. Den,

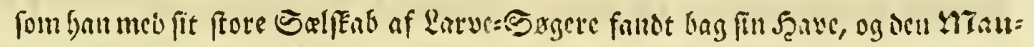
pertuis fisen gav bam, fauvelfom de bam blev tilfentst of busfin, ere iffe, fon han meener, forffiellige, menen og den jamme 2frt. Den Gwide Tuer: Stregpan e $i=$

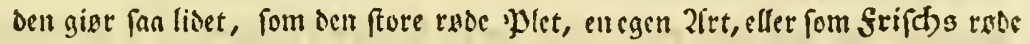

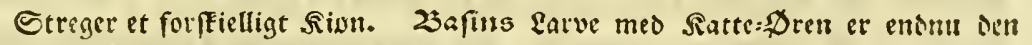

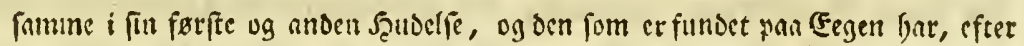

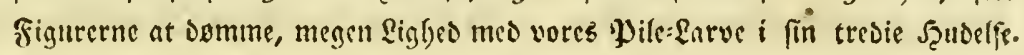
Den fartfe, anden og tredic Gigur pua dent 2 I Ylade vifer Earven i fin Welmagt,

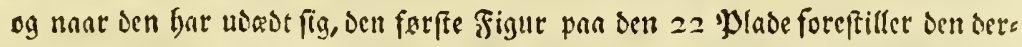

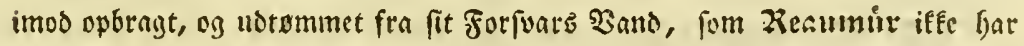

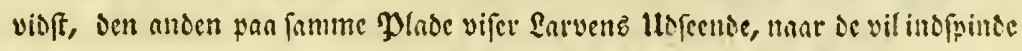
fig og fager efter ₹rer for deraf at Dasme fig fit Szvile: Eted; den trebis, ent fet Figut, er Lawen i fin forfte Ungoom. 
mig en graa ubfrakt og meo fune been til yife= Etammen forfet Minto:

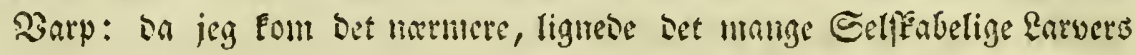

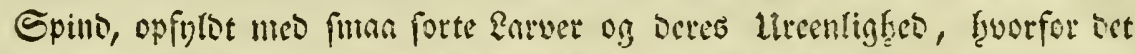
og anfaaes, og i folge heraf foragteses of alle, jeg forevifte Det. Enoe= ligen blew jeg fire Beent waer, en lang Strep og Ninger, og faae, at bet

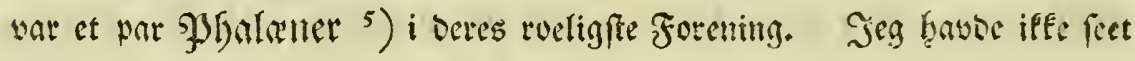

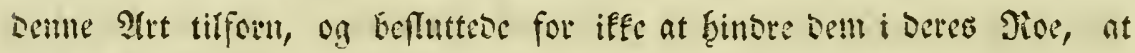

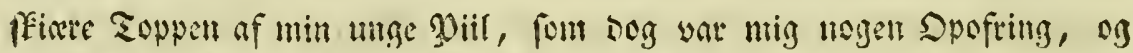
farledes vende jeg glab fiem med mit Bytte. Enfiguer, fom ieg forevife

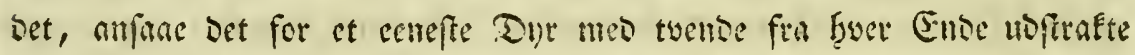

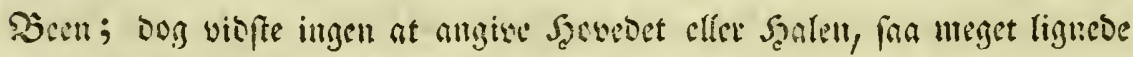

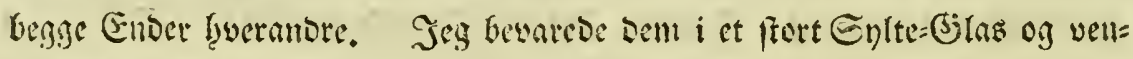
teoe par Dattems Siomme, for at fer sem abffitte. Somblefe nbevergelige

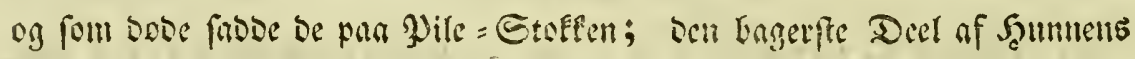

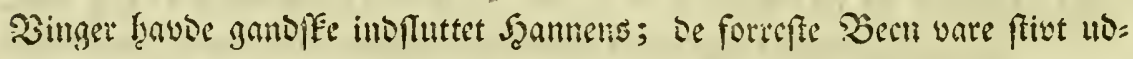

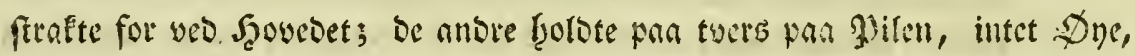

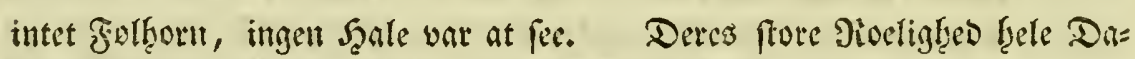

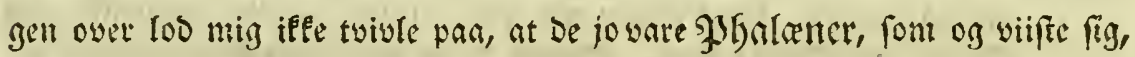

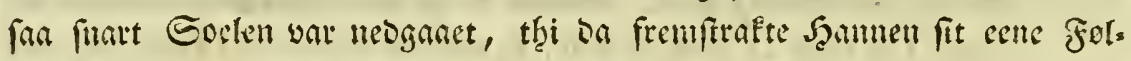
from og forrabede fit Silen. Det uar Den ferfte Sevagelfe, fom $i$ 16

Timet

5) De Gtimlvingeoe Snfefter ubgidue trende Elegter, fom of Tiden, i fuiffen be

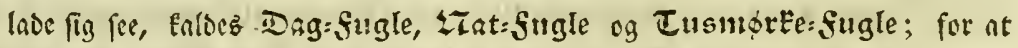

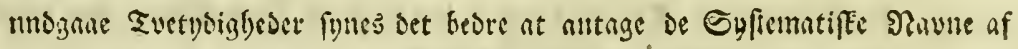
Papillioner, phalener og Gphinter. 


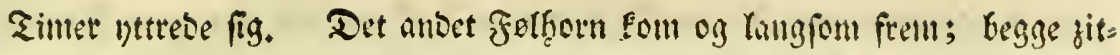
trebe heftigen frem og tilbage; 3ingerne begnnbte at reves; Sanmen fien

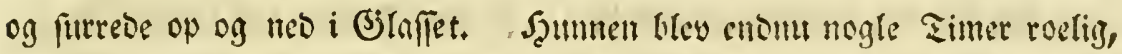

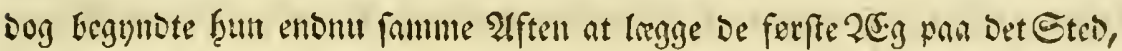

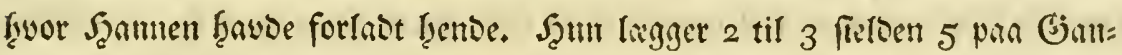
gen og pan et Steo; imeoens Dene Fortetning ganer for fig, bevarger

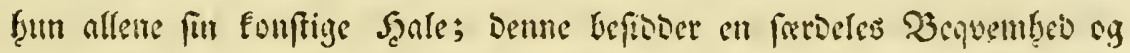

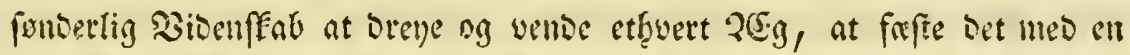

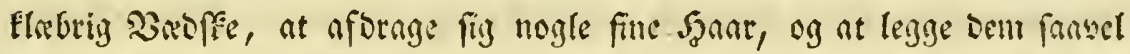
ouen par fout unser 2esget; Dog Femme oe flefte oven par. Darpa

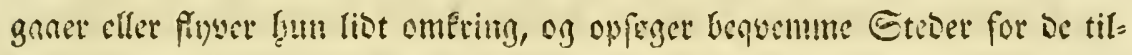

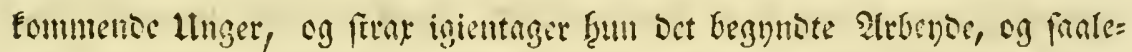
Des afuerlende veobliser at omflyve og at furte 2e g heele Dattent. Dagen ganer fort i fterfte Etitgeo; begge blive pan bet Eteo, hyyor Morgenpun= Den overrmuptcoe sent, Dog byer for fig, og parte fig iffe ticte sllo ens gang. Şammen famler $i$ fin Şvile Frafter til at fortjette Det begunote

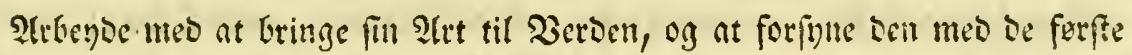

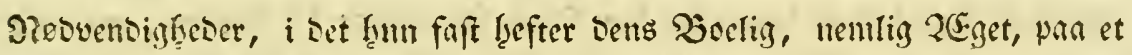

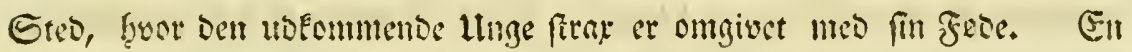

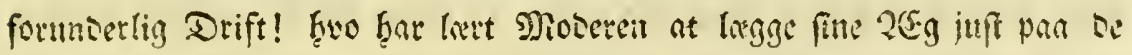

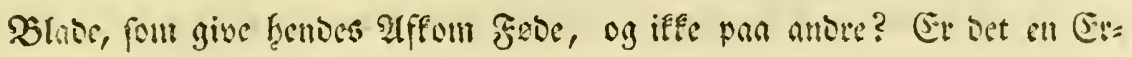
inoring of Den glante og be Bhade, fom ben fetw frar nust til Rivets lint=

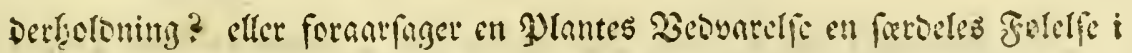
Dens Derver, frem for en andon? eller er Det Rugten fom weyleber? fivor fiin maa ben ware og fyor har bette Sigan fit Eroc.. Ena 
San libet font Natur = Forpferen er $i$ Etand til at angive Stebet

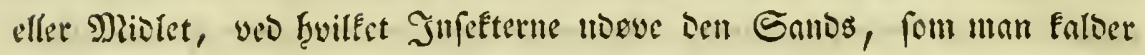

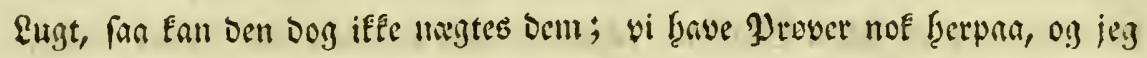

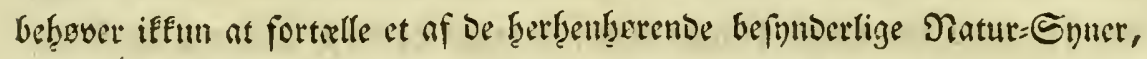

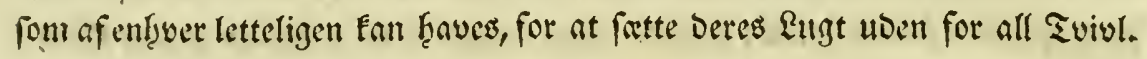

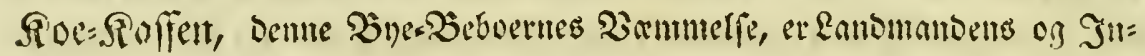

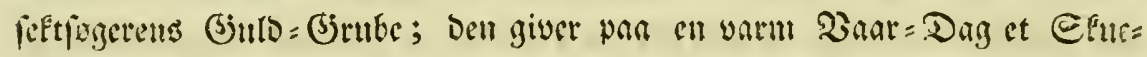
Epil, fom man tilfere med formoring. Soort efter at oen er faftet, til=

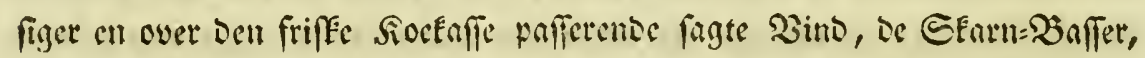

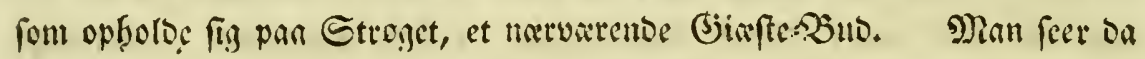

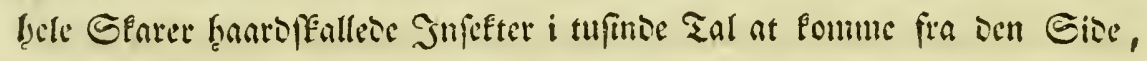
hyvorben 3 intoen blafer og at fige bet Etco, f̧uorfara Engten fommer; De= res Juer og Antal foreges jo netmete be Eomme Nanlet; endeligen for. mere be en Einie, paa 2 til 3 Alen for til, af fuyente og lebende Gree, foum

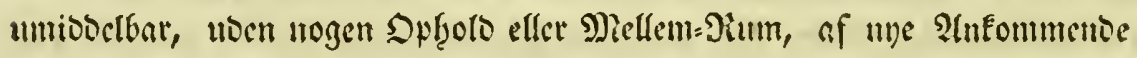
unberftettes og med imegen Siofighted, Enurren og Surren beclsftyrte fing mes

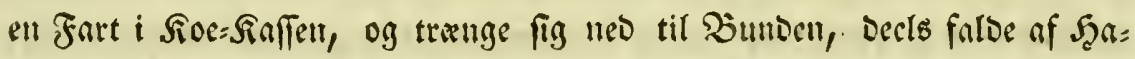

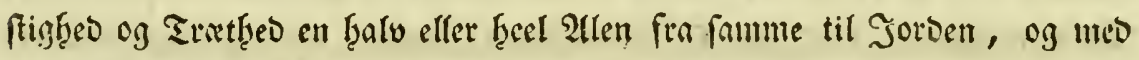

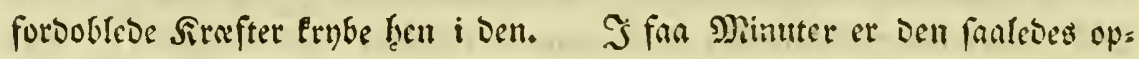
fylot, at Diffe Biefters Samling ubgiur en fterte Siaffe end felv Syerber:

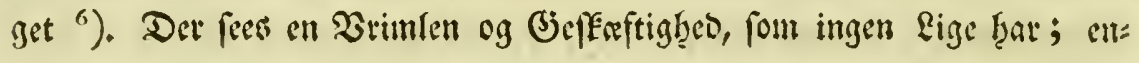
ḩvet

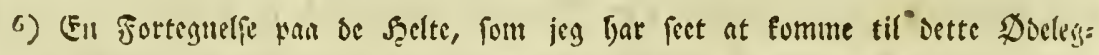
gelisus Bieftebut, vil vel iffe vare ubegngelig: 


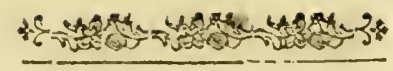

9

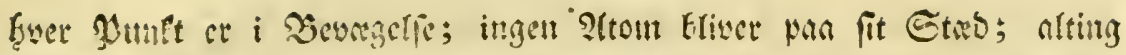

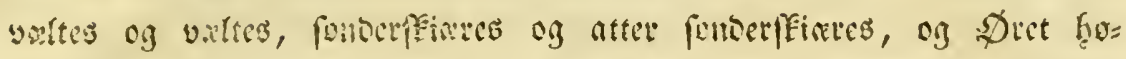

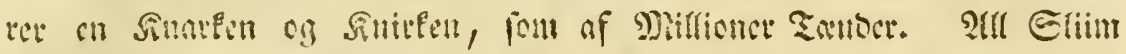

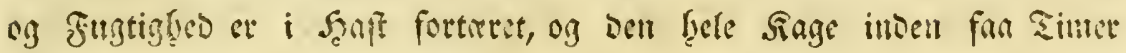

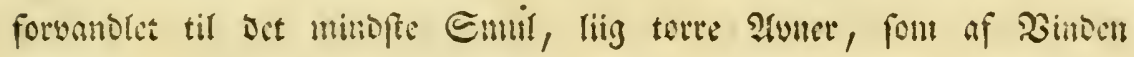
abjurcises.

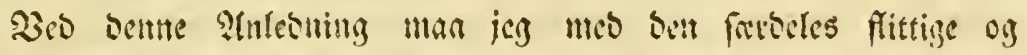

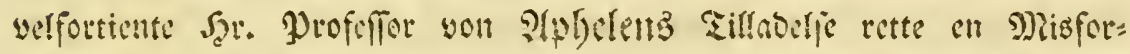

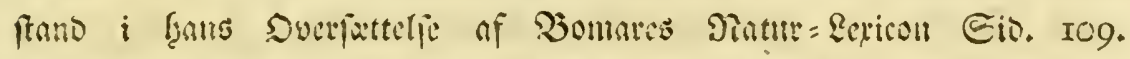

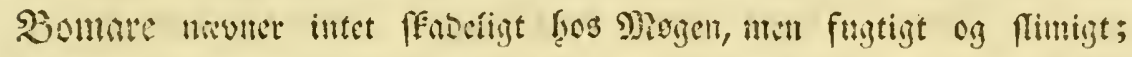

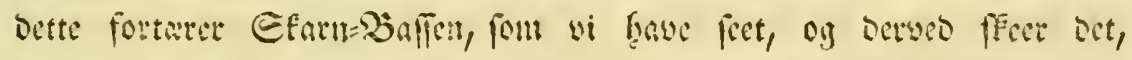

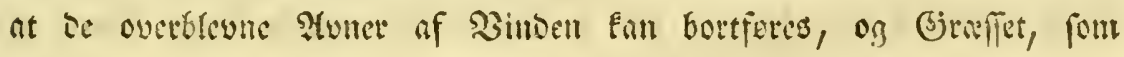
ellers war of Mergen blewen qualt, paa ben fanledes fric bleme

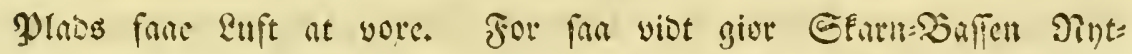

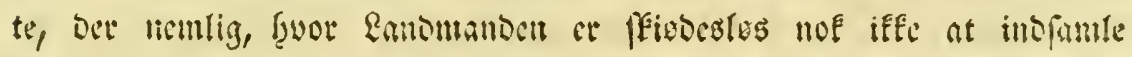

Nagen

Scavabaus ftercorarius ben ftore og ben lille med betes acaris.
confpurcatus.
Copris nuchicornis.
teffulatus.
Dermeftes fcarabiroides.
fimetarius.
Stapbylinus murinus.
bipunétatus.
chryfomelinus.
lineatus.
riparius.
rufipes.
binaculatus mo flete.
lıxmorrhoidalis. 
Megen; men ar semne panpafferioe, fanct EFarn= Buffen intet, Det sllers borttager bet befte, og lader Deri Doune Eamomano Smullet.

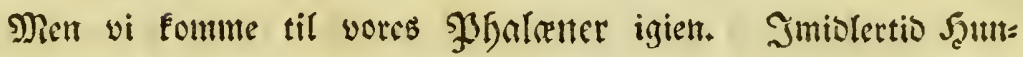
nen allene er befereftiget meo at lregge fine $2 S_{3}$, puermet Sannen

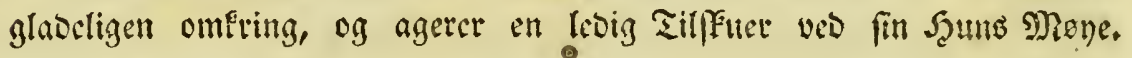
Scan frucs iffe viocte at vere til nogen Sintte, Heo minbre man troer, at fran ved fin Enturen og Smleben on fin Mage, foum ant Dre Fugle med beres Eang, letter f̧ernes 2lrbeibe. Denne Şan=

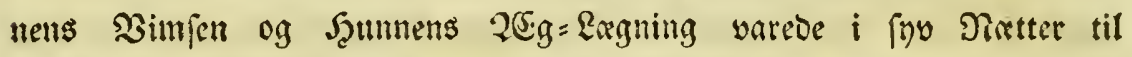
Den 22 Mal. Dagen Derpan Dede Şumen, form Den Der nu ḩay= be opfint fin Tiluntelfes og alle fine Foryanolingers Envemaal. Mon Şannen iefe allerede for 8 Dage haar opfinlot Dette Minal, og Dog lever fan, ffiont afmretig, enomu trettie Dage til betr 26 mod Midong, i fruis Morgenftuno llngerne fravoe beggnot at bryoe t1o of 2eiggene. Er Det iffe noget befnuberligt. Mooeren maa fatte

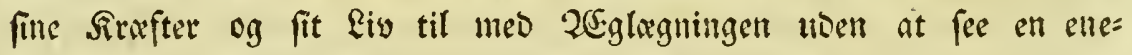
fte of fine Unger; Faberen Derinto feer fig leve op $i$ flere end halutreofinostive Effterfommete, for ḩan Déer. Şyorfor er but anbet

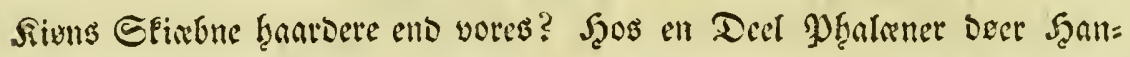
nen frrat efter Parringen; f̧os et Slags Brwsthopper ${ }^{7}$ ) fogẹ f̧an umio. Delbar

7) Bowles, fom befEriver beres Şifforie, figer blant anbet: je vais decrire fes amours avec la liberté d’un Naturalifte, mais avec des intentions 


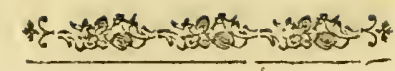

\section{II}

Delbar berefter set ferffe $\mathfrak{B a n b}$ for at Fiole fig; hans $\mathfrak{3}$ inger bli= ve vanoc, llougtige til at fruse og fan brufner. Thefen Det fam= me Peet Degne Sluerne: De partes over Bandene, lase beres 263 fal= De $i$ fanme, og falbe entoligen bag efter, unbertiben $i$ ell fanban

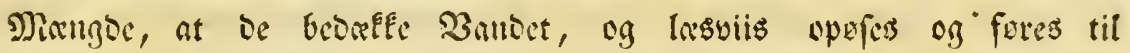

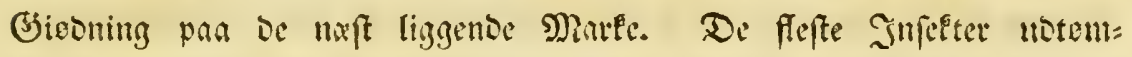
me fig falcoes yed een Worring, at be frat berpan bee.

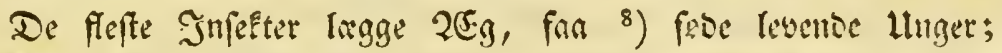

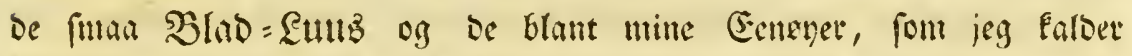
Dopfle, giure begge Decle, bog til forffiellige Titer. De ferfes

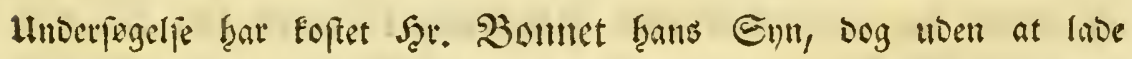
B 2 f̧ant

pures \& philofophiqves, of frtar berpan: ce n'eft pas par des inftans, c'eft par des heures, qu'il faut mefurer la durée de leurs accouplemens. La Langofte jouit dans une feule fois fans interruption de la valeur reelle de la vie entiere de l'homme, partagée en dix mille mo-

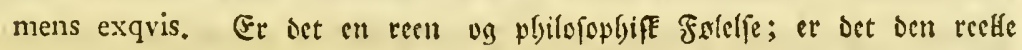

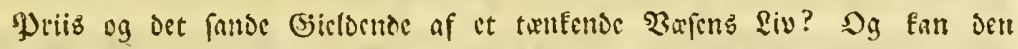

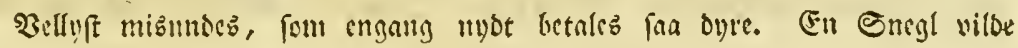
man tilgive set, iffece et Dionneffe, cui vita eft cogitare. Cic.

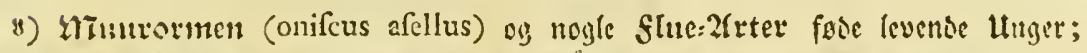

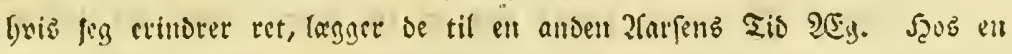
vis Ffue:2itt bar Reaumix benarket, at Maberen beffod af en fummen:

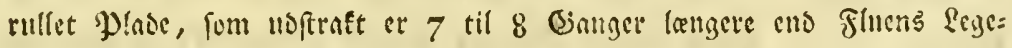
me og ganfe belagt med Fine: Qnever, en wed Sioen of Den alloen, uver s0000. Mem, des inf. tom, 4. P. 415. 


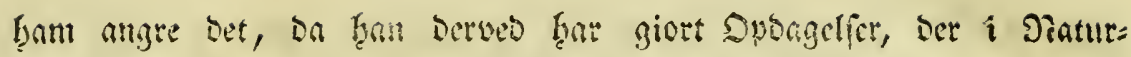

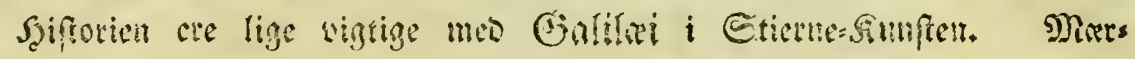
feligt er Det, at twende frore Menos llnoerfegclet af be mobjatte

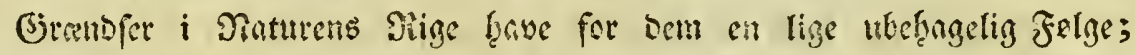

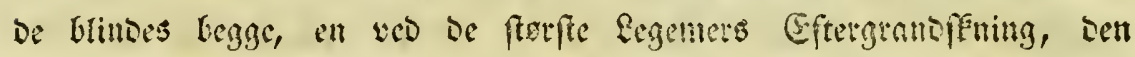
anden wes de minteftes og ingen fortrnter bet. En fanban Eelufor= negtelfe giver (bubs Biemtugers betrogtning, ig en Spofrelfe til fin

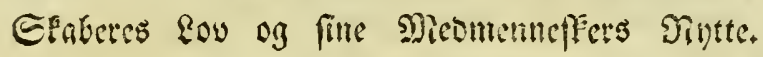

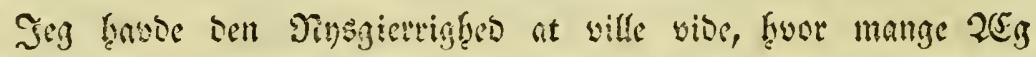
min Sctur fravoe lagt; jeg talte 370 og fan fiefer angive 400, thi

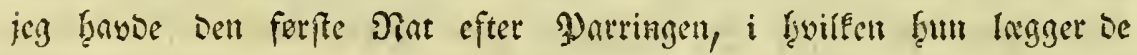

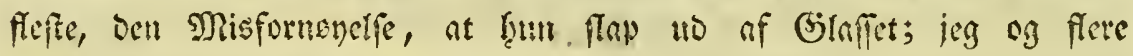

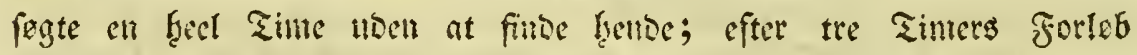

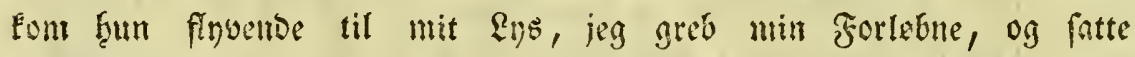

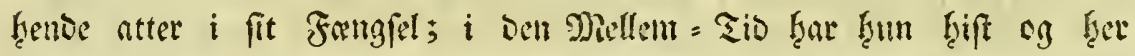
$i$ Sammeret tpatvivlelinen $i$ bet minofte lagt trebive Zeg; ber fantes og nogle facuel paa Dile= Stokfen ntoen for Gilaffet, fom

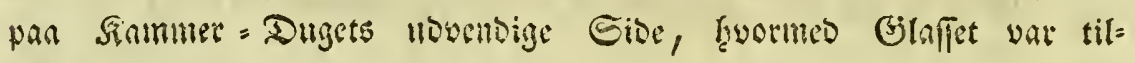
Dowf Et.

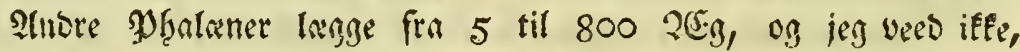

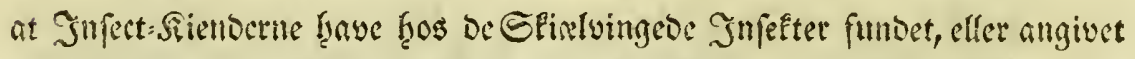
et ferre Tal, Shyor meget blew jeg berfor formoret, og f̧wor libet torde 


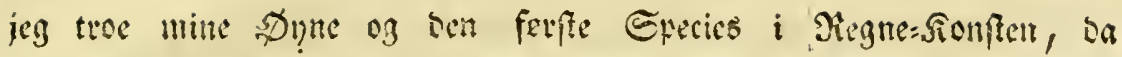

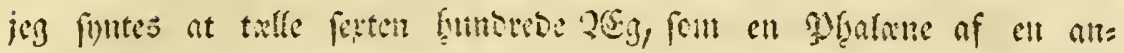

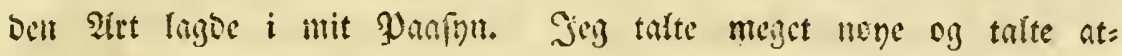
tel, og atter igien, og Sallet hey iffe minder; Det var bell fmut's

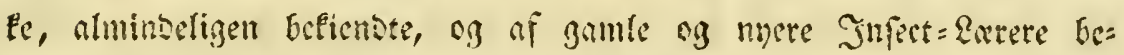

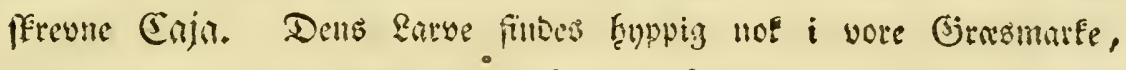
er grandiy, lever lange, entog fra en Eommer til ben anoen, og

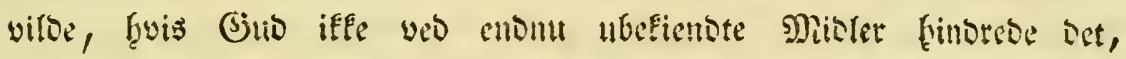
blive Suregets og Det memefferige Sirns Doeleggelie. Et enefte

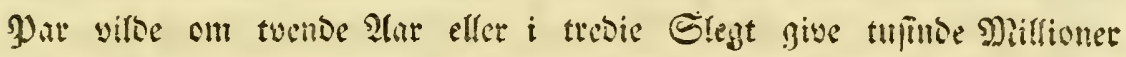

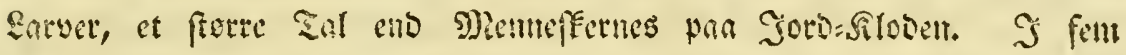

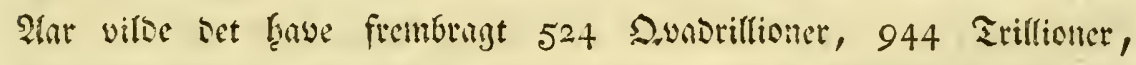

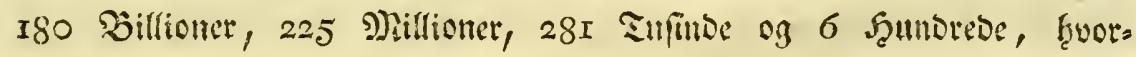
af 524 Duadriffioner og 287 Triffioner $i$ ict femte $\mathfrak{H a r}$ vitoe bes

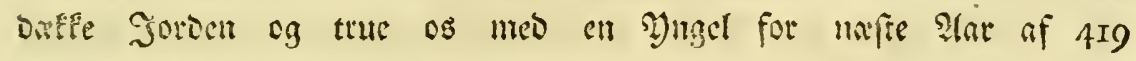
D.vinguiflioner, 430 Dunorillioner og 400 Triffioner.

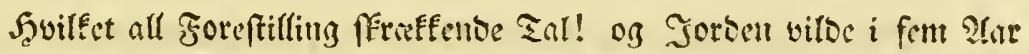
brive en Sorfen, hyis iffe Efaberen, fom f̧ar frembagt seres taltige Sirigg=

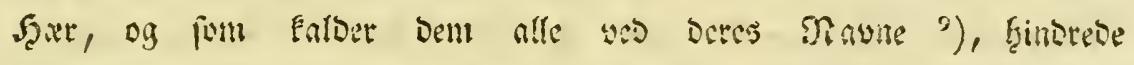

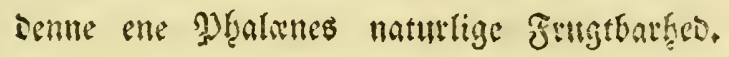

ग) Eịnins 40,26 . 
Den er faa meget farligere, font ben er feilivet, graabiz og

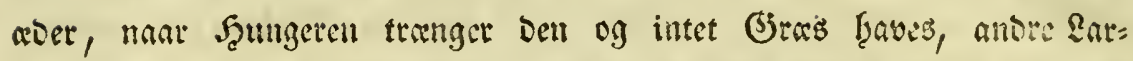
ver endoy fine eghe Brobre; fertil fommer, at Naturen til Forjuar moo bens fiender og Farers Iffuenderfe, iffe allene har erouffet

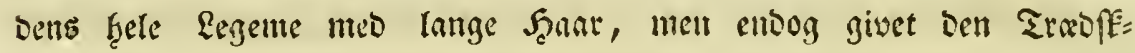
fres at unogare benr. Saa fuart noget narmer fig Det biras, beorpara ben fibder, laber ben fin falbe paa Sorben, vifler fig $i$ et

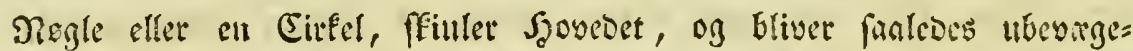
lig, inotil ben troer, at Faren er forlit; Dette ḩar ben tilfalles med mange Rarver og furtofomme Sinferter. Sfte er Brreffet for fullot af bent, at de ved livert Selrit pees at nebfaloe fia Stranet. S Betragtning af Dette, bliver bet en varbiz Bienftano for en $\mathfrak{N a}$

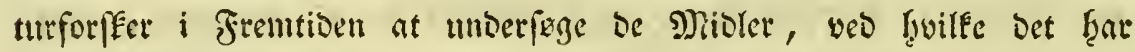
befraget Forfinet at forebugze Diffe Dyts grufounne Formerelfe; mant vilbe derved lobes paa Epor af Mibler, fon nof vil gigre fanmme

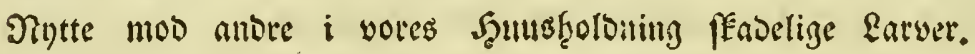

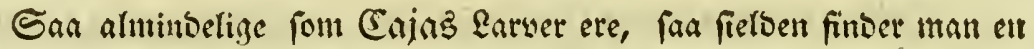

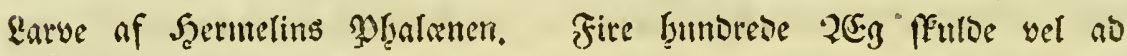
Aare give to bumbrede Scumner, ob biffe firfindstive tufinde Earver;

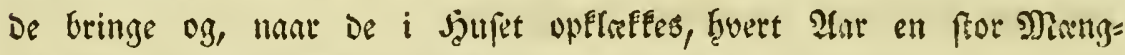

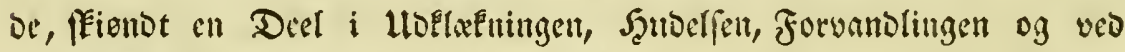

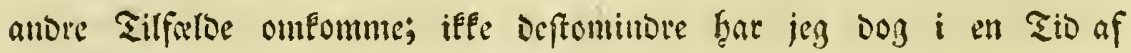
I2 Tar iffe fundet lloen bet eene Par Mgalanet, fom foranlediger 


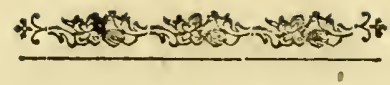

Dette Serift, fire 2Eg og en Earve, wagtet jeg for at feforbre et

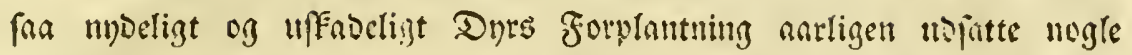
af be $i$ mit Sammer opelrefecte. Shr. De Sicanmir frar tillige med andere frampe Jufeft selfere lenge fegt Dem, og bog hat be ifen fumbet cen og Mattpertutis tre; Ser. Poontet Elager og over

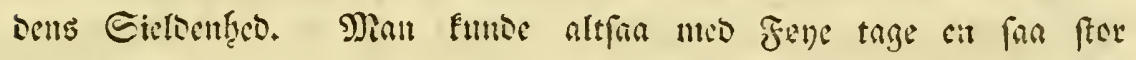

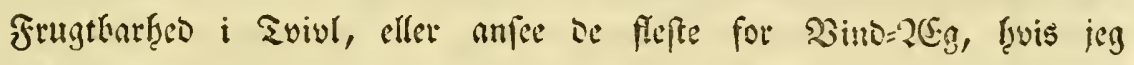
ifle bavoe neve iagttaget, at af et fruert 2 seg fromfom ent leventoe llnge. S S Suertig figer $\mathfrak{S c t}$. De Gieer, at be finder $i$ Miengbe; De una folgeligen $i$ Danntarl og $i$ Franferige have fanoume fien= Der og Forfolgere, fom ifee, elfer iffum $i$ libet Tall finbes $i$ Evers rig. Smod fima Dones Syerlaft, fout 3ejpe:Anter og Flluer, ḩar Natuten forinnet Dem med meere $\mathfrak{B}$ rrge ento mange anbre Earver, men berimod fines be meere utfatte for 3arets Sfarphed; jeg f̧ar iefe marlet, at be noyenfitio fiolde fin op unber Blabene, men altio oyen par og paa Santen, en) ficllet at be inovifle fig $\mathfrak{i} \mathfrak{B}$ la Dene, fou ellers er mange Earve=2rters Beffiermelfe. Formosentli=

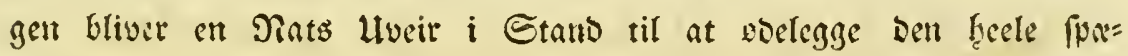
be צfeom. Jeg har berpan et tydeligt Beviis $i$ Sar: ie fire funtrede brede ul af 2eget fra ben 26 til 30 Man; par ienue

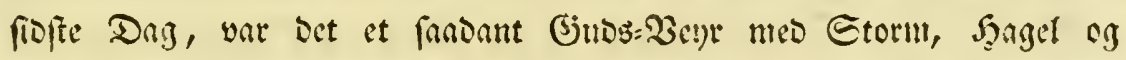
Diegn, at be alle, fyuis be fiavde wurret $i$ frie Rlft, mantte yuret

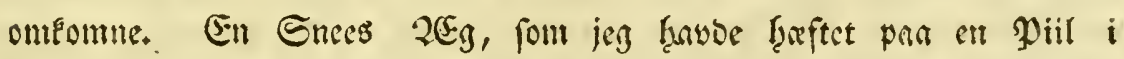

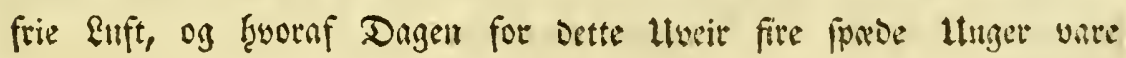




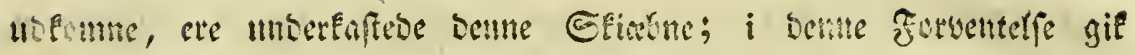
jeg 110 for at fee til Dem; men, byond finter jeg! fyuor flet phillo

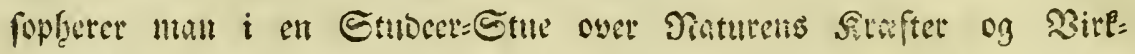
uinger! Safe grumbig finnenbe Elutuinger, igintagne Sommeteffer bringe os paa Epor af Daturens vife og fante Samudfifoloning.

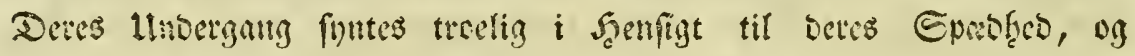
neovensig for at gotgiare \$langelen pan en fan frugtuar slut; Dog

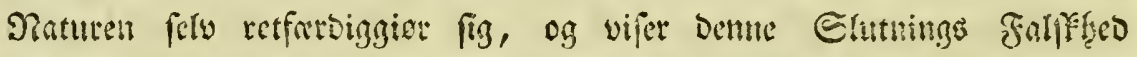
noen at fige os ben fanbe. De fire faroe sarver vare iffe om= foume, men $i$ fire Doges beftanbige Storm og Stho sorte og muntre, eg vesbleve at iile til Deres Futsentelfe. Shvornf Eommer

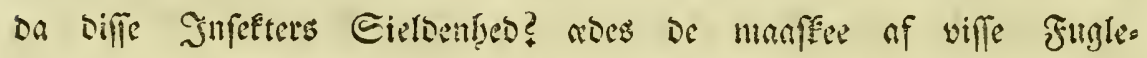
2rtet, cller - Dog Tyyad sil vi plage os meo uftere Sitetninget, vi vil beller oppefie an lnfferig Bumarkele, og imblertio prife EFabes

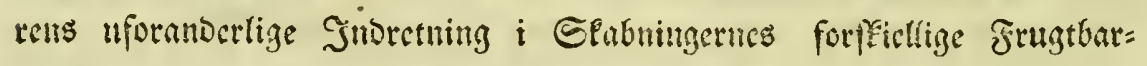
freo: jo mittore Dyr, jo overfebigere affent, jo minore Mranter,

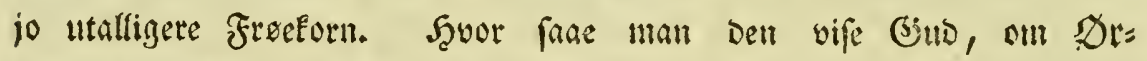

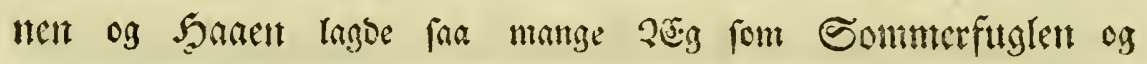
Silben, og biffe far faa fom bine; vilbe iffe line eselegge $\mathfrak{B c r}$

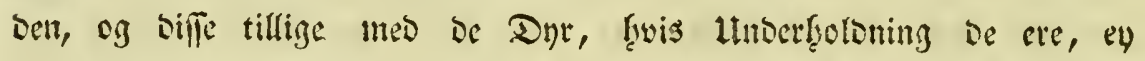

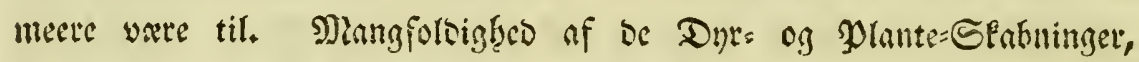

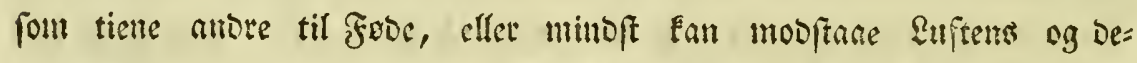

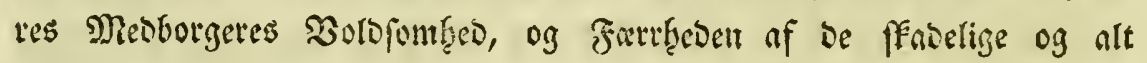
fortirembe fiemvife bell Margtige og Gjobe. 
Fer vi begive os til se fore forvandinger, fout bifle

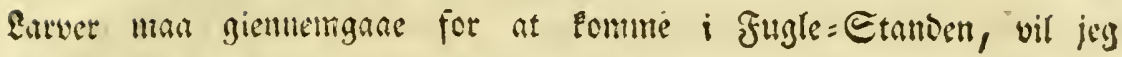
forteligen beffrive deres freters liofentide.

Ducroingertle ere Tyuibgraa, tegnebe med 2 til 3 Piacer portagtige Etrejer i bialve Elipfer, og entiven meo en fort Dianb. Fra Deres Siod. ULloke tre brume Etreger, fom Earer; par. famme Sted fees nogle forte Priffer. 11mber=\$3ingerme ere gra, og glat=-

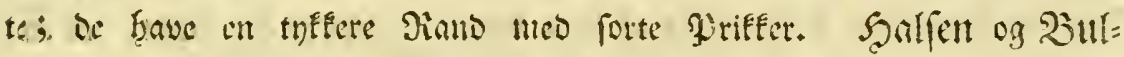

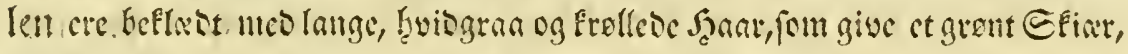

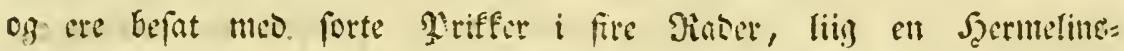
Sante meo forte Saler. Womment et ouen pan belagt mes faside

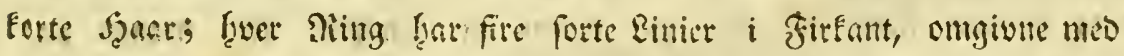
twende fpios!efienbe forte Mletter; Benne Segning. Far givet Ben Naun.

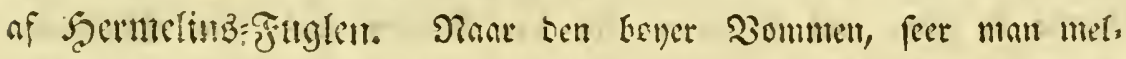

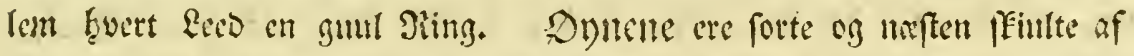
Syan. Fra Panden neDganer mellem Dynene en ḩvibhaaret Snip; neben for Benne fers twenbe brume Epibfer of en halv simies Eanger; Det vil blive vanffesigt at beftemme beres Bang; mon be

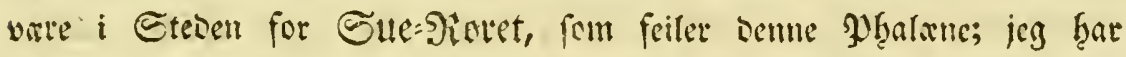
iffe Eemarfet bem forend efter Gnfetets Deb. De for Seent ere,

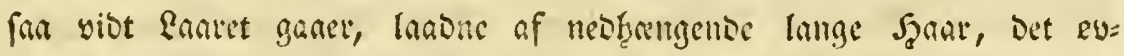

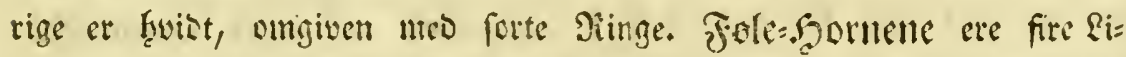

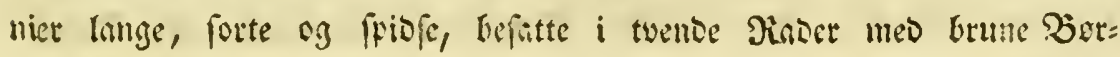




\section{I8}

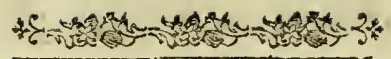

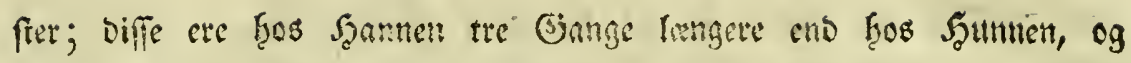

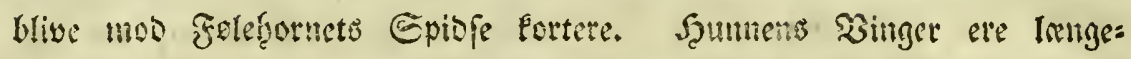

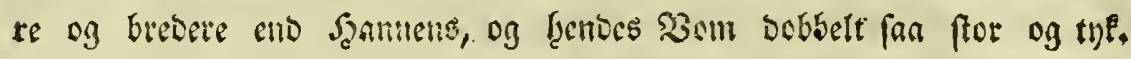

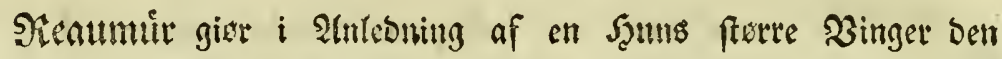

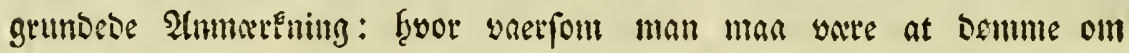
Burten. of Dyienes Eemmer, frafom enfruer wil troe, at Singerne.

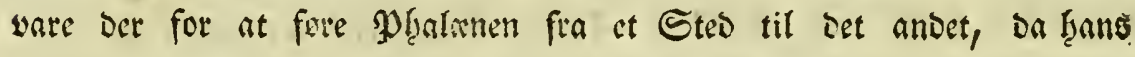
Soun boz ingenfinde betiente fig of Dem bertil. Bel et bet fantet, at en Deel factuncr iffe finve lnutgt omfring, men gemeenligen, i far for Sartingen frode par det Eted, fyvor be af Puppen ere nofomme;

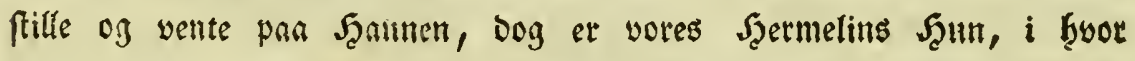
tuny fendos Sirop cli, ifle faa boven og langfom fom mange ans Dre Pryalener, og frendes Binger, en at nawne den Poryderfe, og 3arme be give, fom Naturen ifle haar forfumt f̧os fure Slabnin

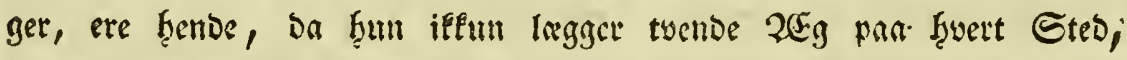
fygyligen noovendige; fyvor manne Turer ma bun ille tage for

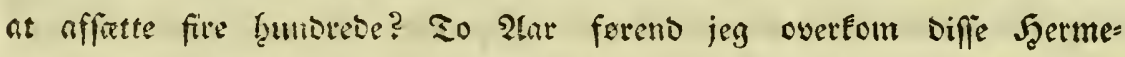

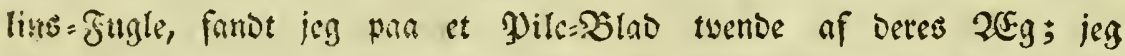

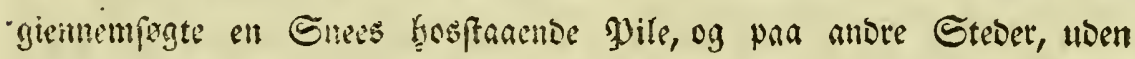
at finto ellom et, f̧uilfet mefom bevifer, f̧yor vibtleftigen og lanjt

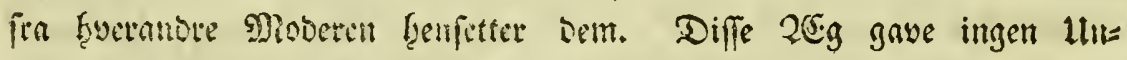
ger, og lose mig for sen ₹ib i lividenhed om deres Sprimberfe.

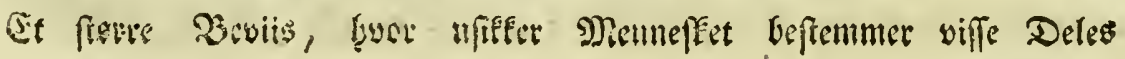

Sbutig 


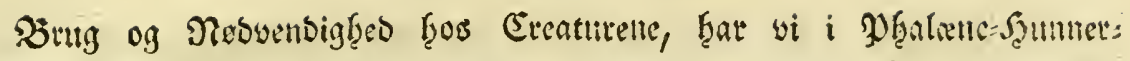

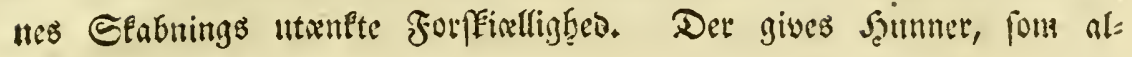
beles ingen Binger b̧ave, allenefte et lidet Spor til fanmu: andre, fom imoo Natumens alminoelige on Millionergange fulfre Fiegel bes

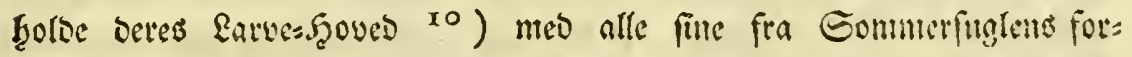

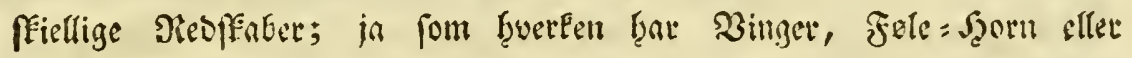
Been, ${ }^{x}$ ) men efter alle giennentyangne Forvantinger elrom bes

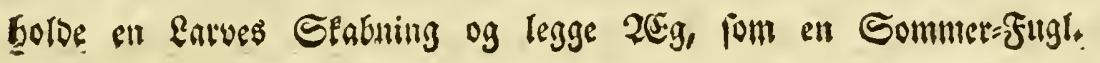

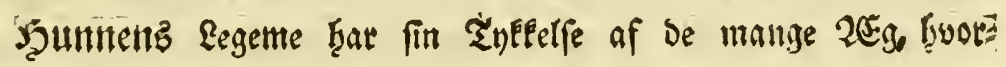
med ben ex opfylot; det laber fom sens inderfte beftos af bare 25ig, ligefom ben og Pommer udaf fit Frnglel, og lever fan Dage

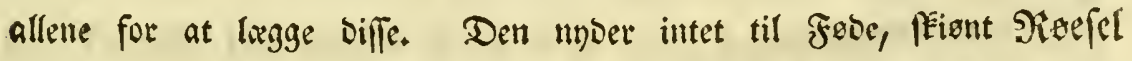
anfeer bet for en Nobventighted bos ben at fpife; Den baur Derfor ille Fellet noget Sue:Per, fon Papillionerne og en Deel golgatener,

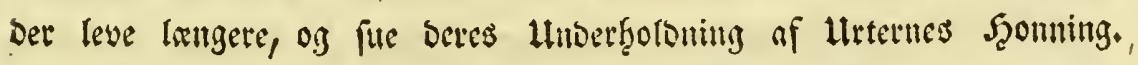

Regemets inducrnoige bjefralt og anatomipfe Deele vil jeg iffe beferive, fioen be inteteffere bet mintefe Tal af mine Eafere, oa do ¿ 2 res

xo) En faabat bar jeg beferevet $i$ memoires etrangeres de pacademie de fciences de Paris, og anfort $i$ min Fauna infeat, Fridr. p. 73. under Savin af Phalena hateroclita.

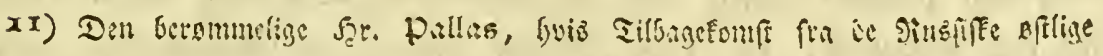

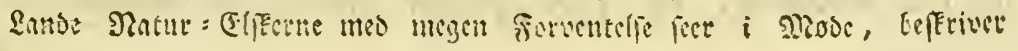

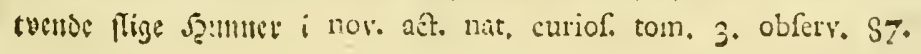




\section{0}

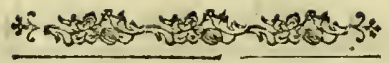

res Uluberiogelie vitbe fofte en Deel af biffe mobelige Earbers Da:

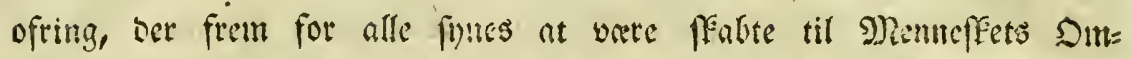

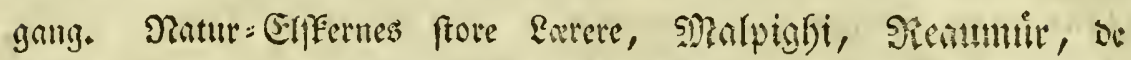
Geer, og nyligen Sufefternes forfte Stuatomift Sat. Enontet, fon

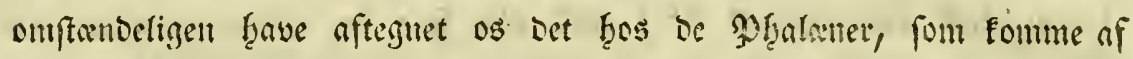

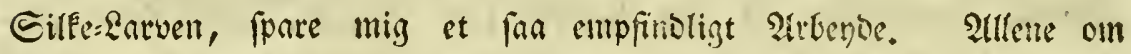
205gene vil jeg meloe noget. Man wect, at be fralebes inotage

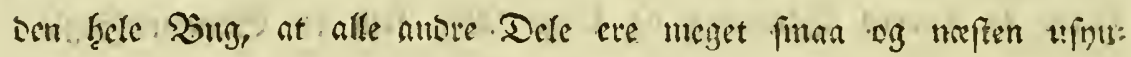

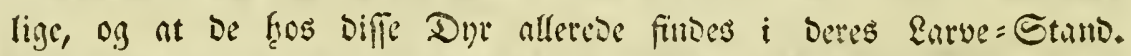

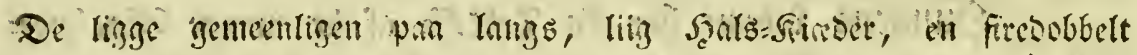

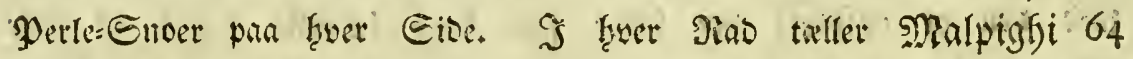
og Srr. De Geer 60 til 70.

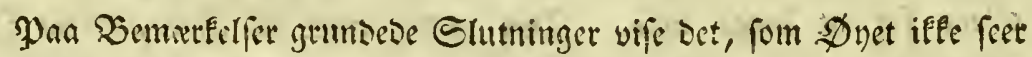

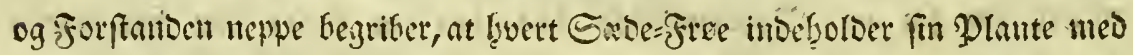
Gitene, Blade, Blomfter, Befungtelfes Eemmer og bets Millioner paafolgen=

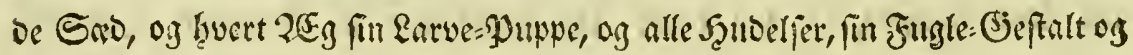
400 til 1600 2Eg; biffe ere alfercose b̧os Earven inutige og vente igiennem Dens forvandlingst at iffamefrettes til at befrugtes og $110=$

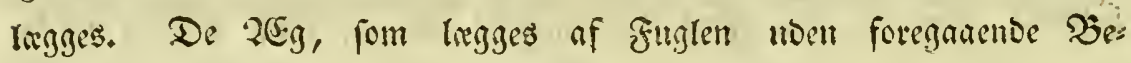
frugtele, frembringe intet; oe tabe i fort Tio beres Srindreb, og

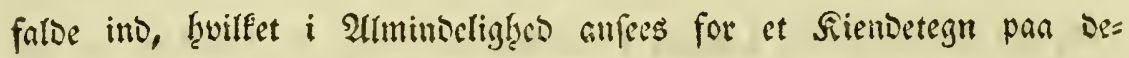
res Iffrugtbarfieb; bog fiar jeg funtet at Efpe=Rarben med Scomet

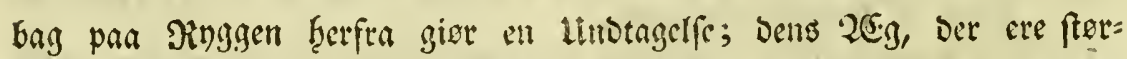

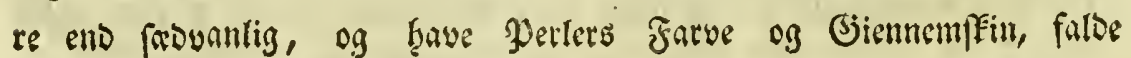
into, 


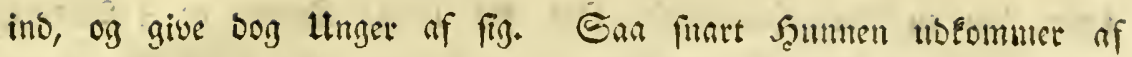

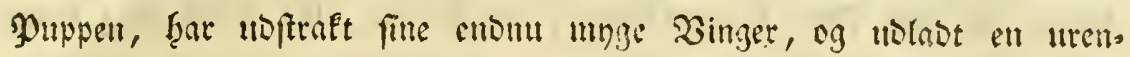
lig Brolfee, er fyun frar $i$ Etanto til at tage moo Sacumen, og umibselbar berpaa at lregge fine 2Eig; inbfinber fig ingen sean, bli= wer frum nogle Dage $i$ forventelfe, og enbeligen labe bem fra fig med famme Simforg, par fanme Slabe og i famme Sorten, fom

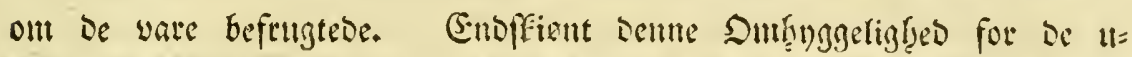
befrugtede 2es.

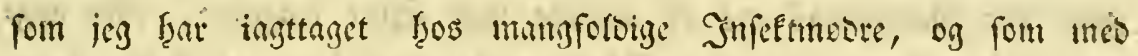

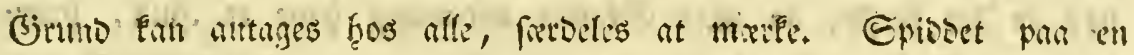

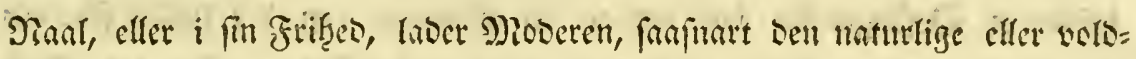
fomme Ded nermer fig, fine $2 \mathbb{E}$ g eller leventibe lutger fra fig. Entoog mifroffopiffe Dre frobe beres 2Eg og Lunger fra fig, naar 3andets Llobumftning ttuer Medernes lnoergang.

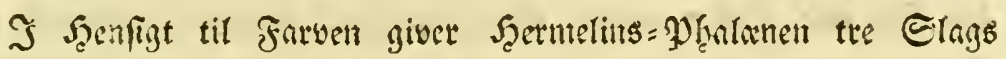

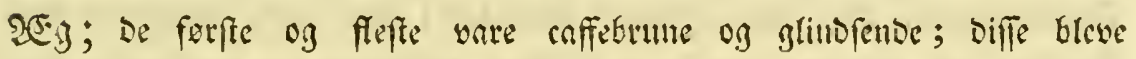
og be farfte i liolrgningen; mange sare anfigtfarvebe, meget faa

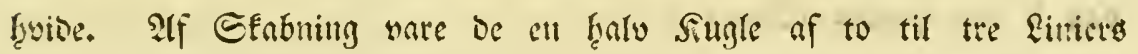
Etortele; oven pan $i$ Mibodpunften fanes en lisen pril af smeen

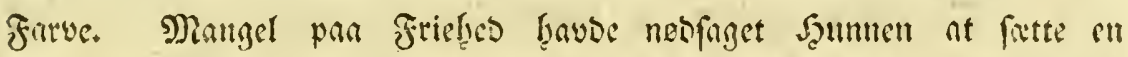
Ded 2erg pan Bjluffets inbuendige Eise, og gav mig aluleoning

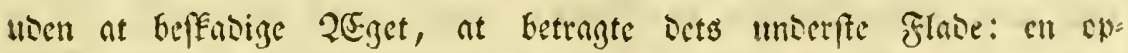

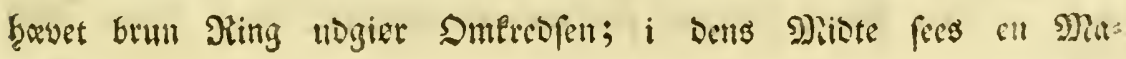




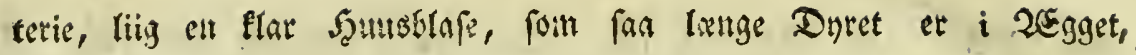
laanet af bets forte Eegeme Den forte Farve. Et ledight 20.3 bragt unber Forftertelie= Slaffet viifte nig, at SFallen beftaacr af twenbe Seinber; Den uovendige, fon er glimbfente og meget tymo, f̧avbe ved en Shanbelfe givet fig fira Den inboentige, fom er lyfere og matt, og med fine mange forbybelfer og Dpferuelfer forefiller at Dlet.

Dette bragte mig paa at parge: i f̧uad Tilftans ete 2Egs= gene for Parringen? ere de allerebe meo Sfal omgione, eller nogs ne? er bet forfte, ba maa Sfallen nobvendig have et Scull, f̧vor: igientmem Sefrugtelfen Ean trange til Embryo, og Det lunde fiee en= ten igiennem Den $\mathfrak{y}$ ril, foum fees oven paa 2Eigget, eller Det Şull, fom neven under er besuffet med Den flate Şinde; er bet frofte,

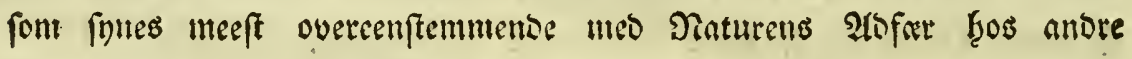
2Eglreggende Dyr, og med be fime Syutler $i$ ben netformige Shimbe, Der fra alle Siber inofue Befuanglelfens 3abfere, farlebes fom tios Jilferestaunen, Det, naar Scumen frar labet Den fare, overgybes med Melfen; Da fperges: ved Gyad Mibdel illeflrebes be umiboelbar ber: paa $i$ ben haarbe Slall?

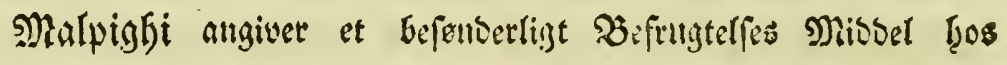
Whalenterne: Gaut hat funbet paa ben ene Eibe of 2eigge = Dierene

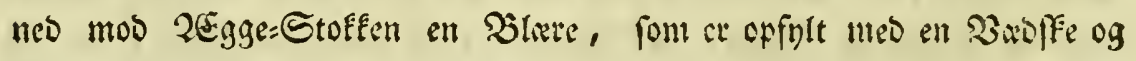

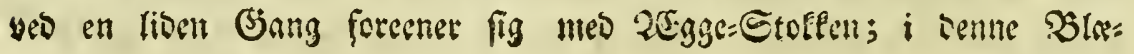




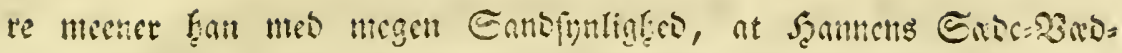

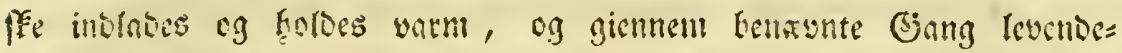

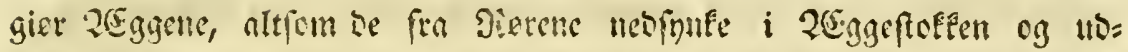

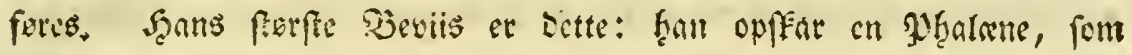
nyeligen fravbe varet partet, og fanct, at be 2eg, fom vare $i$ Tx: rene og enonu iffe pafferebe forbi benne bjang nes $i$ 2rggeftoffert, insermmpise fig i Ruften, fom sores ovenanferte Indagelfe ungtet;

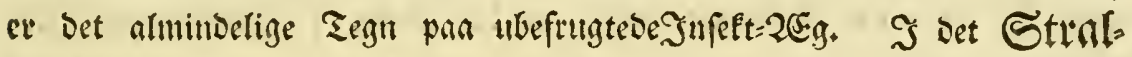
ftuldifte Magajin forefonmer nogle tine Bemarkelfer, fon beftnrle Denne Dicening. De 2ङg, fom en befwangret Mober laber fra fig; Gave en anden Farbe, ent be, fom gaae fra en ubefuangret, ellec fout flibes tib af en befonngret; altfan man 2eggene foranore beres Farve $i$ bet be paffere giennem 2Egge= Stoflen, og ben Forandring maa be fane fra sen Sabe=3roffe, fom bevares i omtalte Blrete. Dette farter at meget fittorigt forfog thoaf all Tuivl: Man hat tinget en

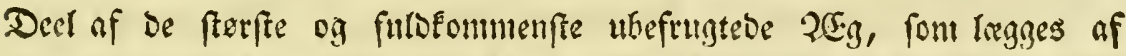

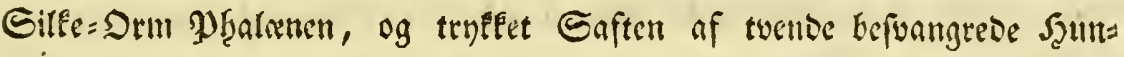
ners Slacer pan Dem; De vaabgiordte antoge en Glane Farve, ligea fom be noturligen befrugtede, og be, fom ingen 23 roffé fil, og fols geligen bleve ubefungtebe, bef̧olote beres gutule Farve. Dentue fon= ftige Befrugtning fortiner at igientages, og at tovibes, Man vil

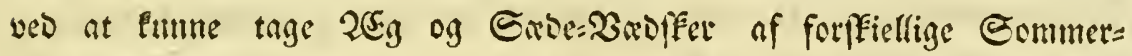
Fugl=2lrter frembringe Bafterter blant Jinfettum, fanlebes fom Şr. Rơlreutet har frembragt blant Planterne, og fprebe et nut ans 
oucr Sturings=3retEet. Dette wil blive far meget vigtigete, fom bet

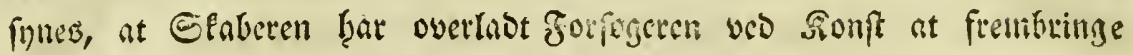
fanonme Efabninger, fom naturliguits iffe vilbe bave erifteret. Seg frar ingenfinde fundet formane tornde Commerfugl=2titer at parre fig fyyor friofing Sammen end finnes at vare; jeg veed en ficller, at ans Dre Gave varet mere Inffelige; vel partes Papillionerme Sutritia og Jantra $i$ beres frif̧eo meo f̧ucrandre, ment Deme Parring har, Iangt frm at give Saftarter, wiift, at be alleene $i$ Sitonnet ere for pitullige.

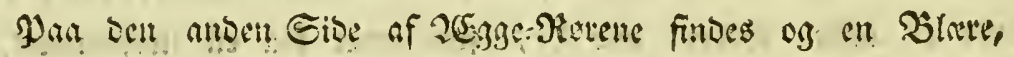

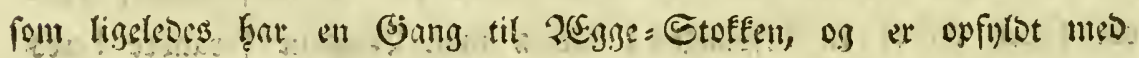
en summengtig Eaft, hyis Srug iffe angives. Efuloe iffe benule

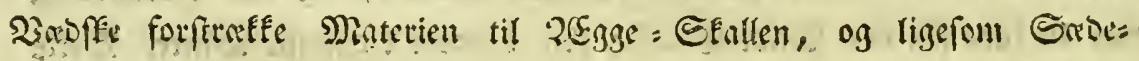
3adpen fra ben ene Sibe trangede i Embryo og oplivede fammi, Da frap fra ben anden Eibe ongive Fofteret til beffirermelfe og.

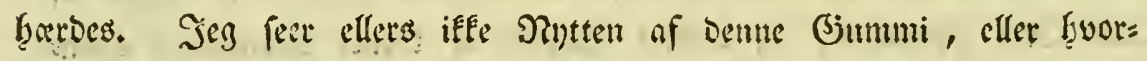
fri Materie til Şallen vil tages. Efallens Senfgeo og grinofente. Blatḩed befturfer ifle libet Denne Meening.

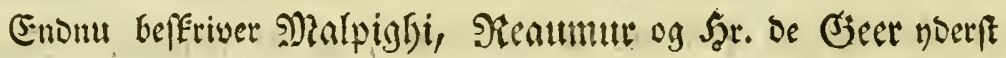

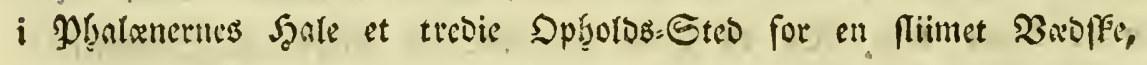
giemem Egiffen 2Grggene paffire, naar be gaae ut af 2Egge= Stoflen.

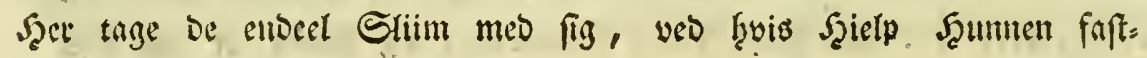

eliner 


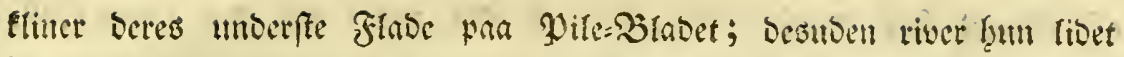

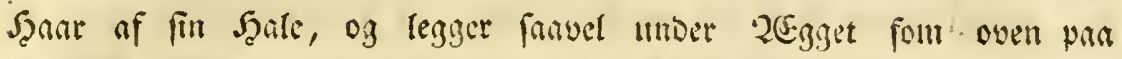
famme. Sendere pleye gantofles at overeline 26riggene med ent tert

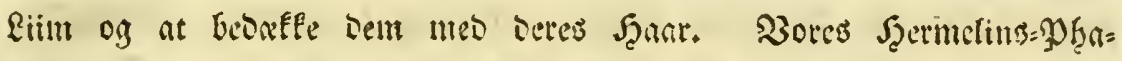

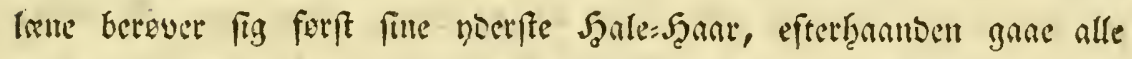
Dinggens, Butgens og Butuftets $i$ Rebet. Şyor naturlig of fivor

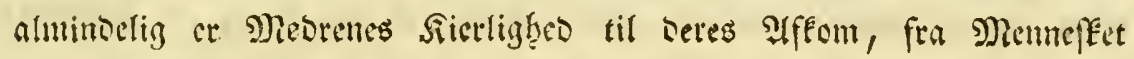
til Sommerfuglen!

3i frave feet vore Whalener i beres futhe Wunt, mesens

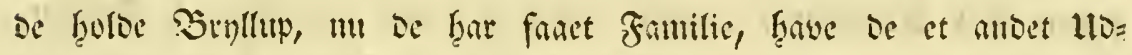

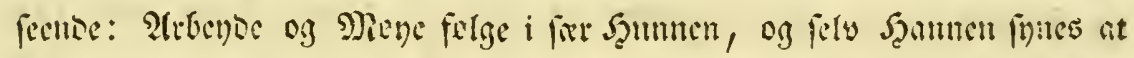
give fin Silroning til 2eggenes bevaring. De fumffe Singet

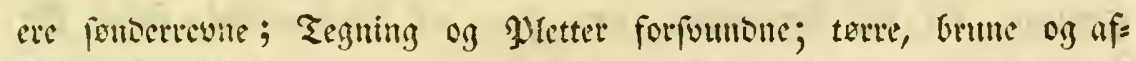

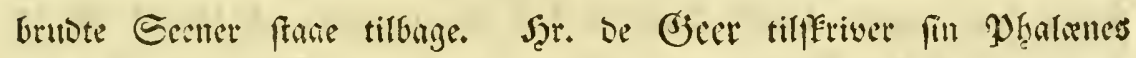

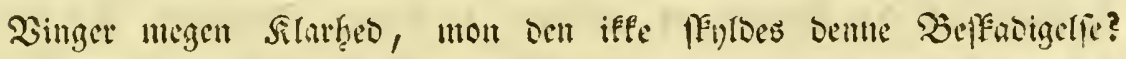

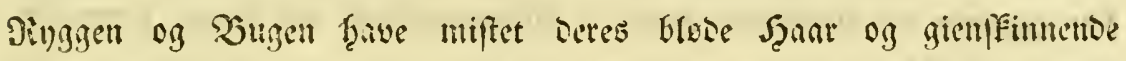

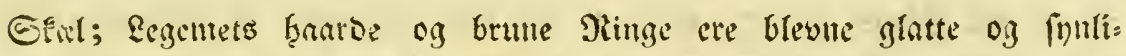
ge. De thave givet fin til Drits for beres Unger, fom Deres firt bre for bem.

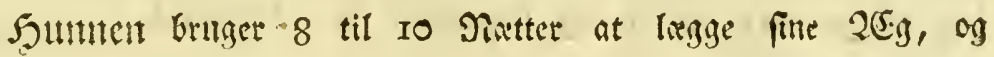

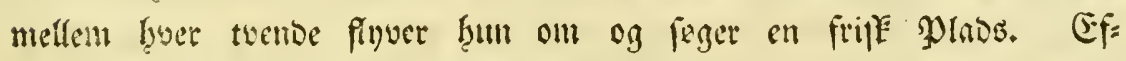

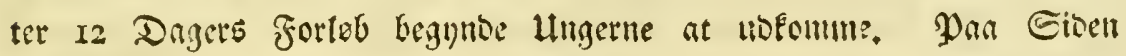


of 2ergget fees ell fort Đrif, fom er iffe anbet end Det farfte f̧ull, foul Lingen borer med fin byerfe Famb, og fiar set forte Enn af

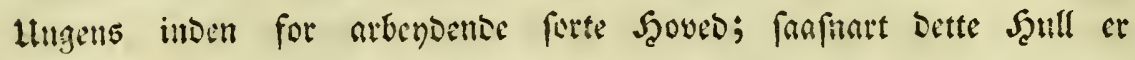
giort, fefter sen be allore Fimber faft, og fegunter at lasbraffe; jeg ţar nogle Bjanger yco Şiclp af en Eupe tilfeet bette Iftrende;

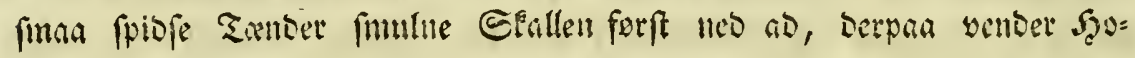
veoet fin og fmulner op ab, indtil Şullet it faa frort, at Scovebet fan gane igiemmem Det. Sinaer bette all, ba fan Siroppen, foun er

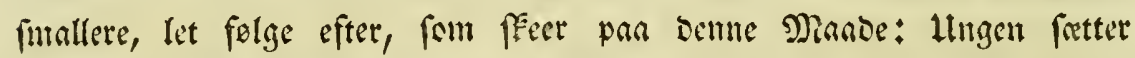
fine twende fortefte Been faft $i$ Efallen, og brager en Deel af $\varepsilon_{t=}$

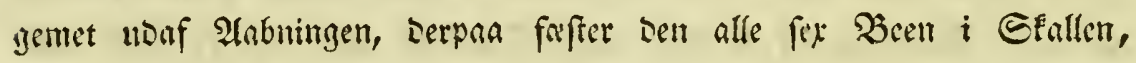
og faleses felget fieele Regentet og Syalen. Llsen Tuibl aber sent frrap be Emul, fom ben, for at givere fig en Hlogang, gnawer af Efallen, thii jeg f̧ar enoog wed Shielp af Forfterreljes = Blas iffe fumbet Det mintife Deraf; Destiden far jeg marfet, at Hingen af og til fretter of fra Sallen, og veobliver at beverge Sarmbeme, fom om

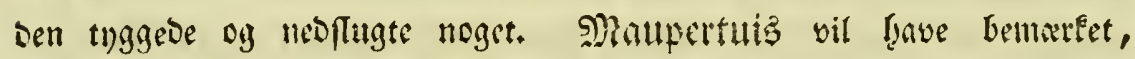
nt et wift Slags Rarver, efter at be ere Uiggngne af 2erggente, ofte selloe, tillonge for at gnave Dem, og Deri $i$ en $T$ id af twende Dane

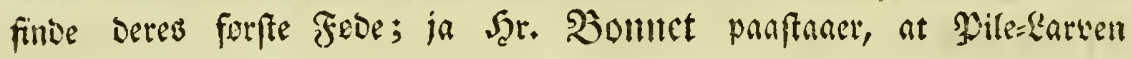
forteret fin Şam med Pandeffal, Tander og Baen Fort efter Dem frar aflagt Den; f̧wilfet jeg ingenfinde f̧ar bemirefet. 


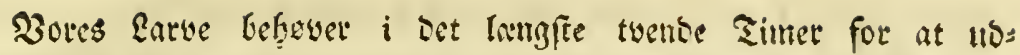

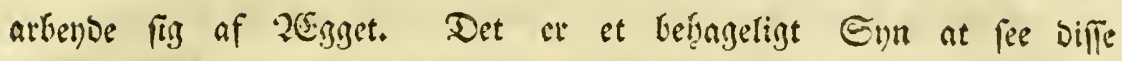

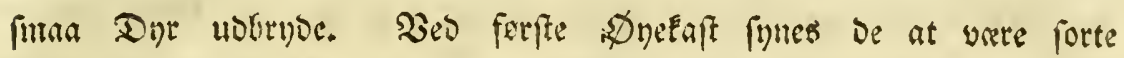
Uteenligf̧eber; men man Ean el) anoet eno forumbres, naa: man feer ben Munterfeco, meo fyilfen bette forte bevorger fig, og at bet

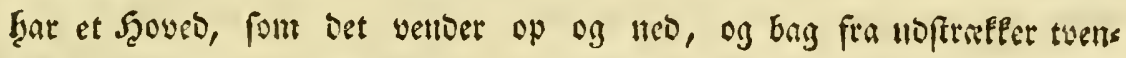
De Nopentreroe Trevolet.

Mian fienber mu et fort Segente, fout er fantutrebie Sinie

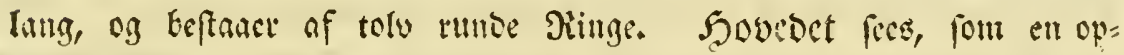

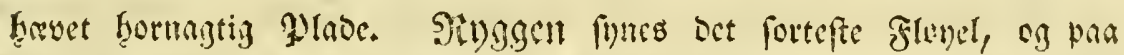
fyuer Siing fers veo Shielp af Enpen tuenoe lorte Borfter. De trende for

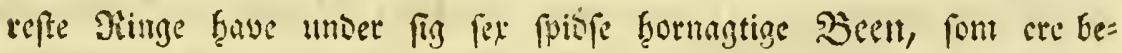

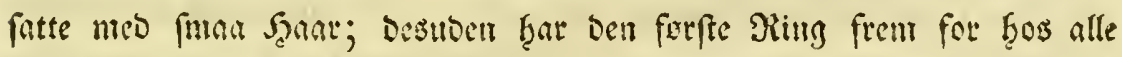
antre mig befiente garver twente befinnoerlige cyen pas yerff ntoo

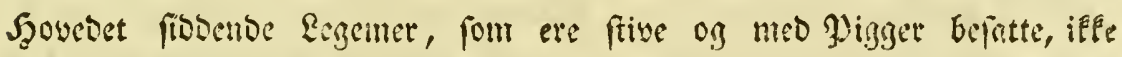
ulige Snappen par wore 3nigteres Miorgenfiemer, og nefter fan lan= ge fom Senene; be frampe false ben uegentligen Drene og fore

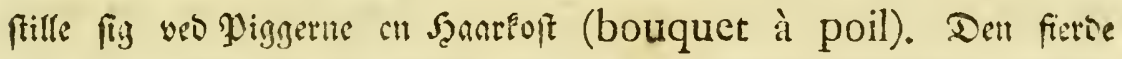
og fente Ding cr, fom hos alle fertentenede Earver, Hear Bien;

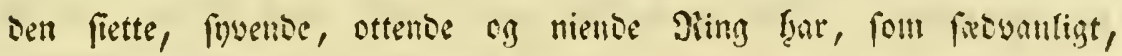

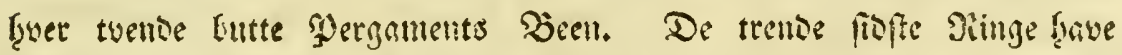
ingen Been, og free viger Naturen atter fra bet Stmintelige fros

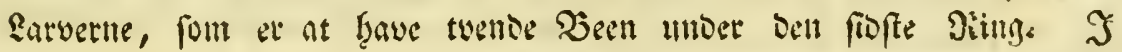


Eteden for diffe ubgane fra Enoen af wores Wile= Rarwe tombe lange Spibfer, fou frox falde i Donnene; beg fan se yel iffe fones

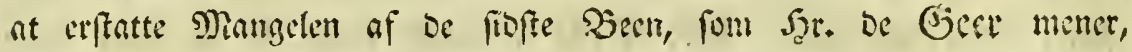
cller at bielpe sarven $i$ fin binng. De cre at anfer fom twenbe Sfeder, ber fyur indefrolde et Epyo, fom Earven efter bef̧ag fan

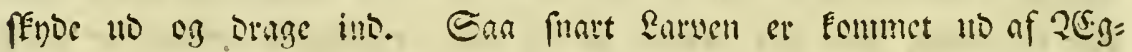
get, er but bens forfte Foretagende at bringe seme llormftning $i$ brugbar Etamd; i 2Sigget ar Epyout noen for Eferen, trente bange langs meo fanme frem og tilbage benct, fanfoum det lloftraft

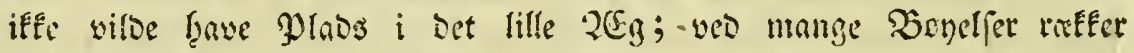

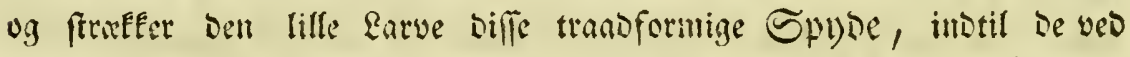

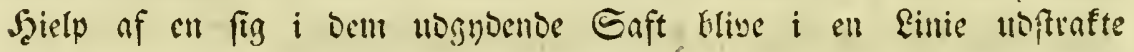

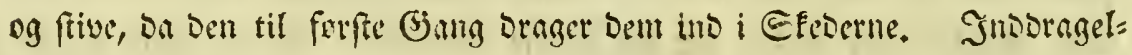

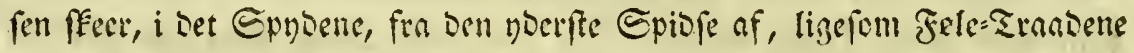
hyos Eant: Sneglene, orage fig ind i. fing fetv, og labe i Endent tyoeligen fee et Şul, i fruilfet Siaterien fra Eiberne inbfluber. Lloftraft ere de

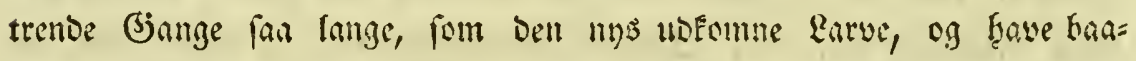
De Epro og Efede ved Deres frerfe fremeomit en Siofenfarve, men Epeben, Der for at fumne romme Det inbuendige Epyo, er faa meget

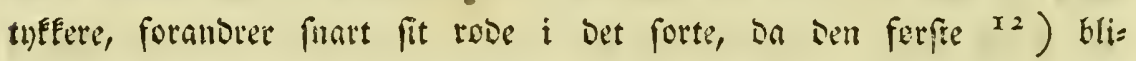
yer uforandret. Efeten ar befat med meget fima Poigger, og er

fanvel

12) Sammerferte de Geer figet vel, at biffe Epyde bos be unge Eatwer cre

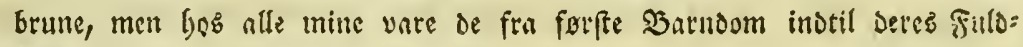
funmenged ufornnoerligen Tivenrode. 


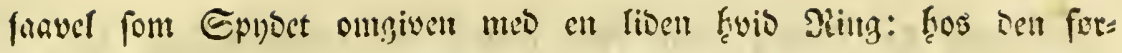

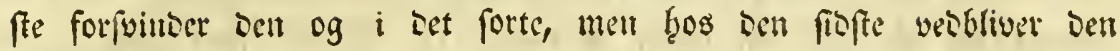
Eienoeligen.

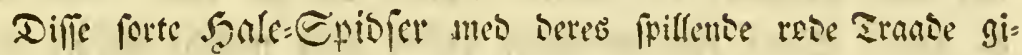

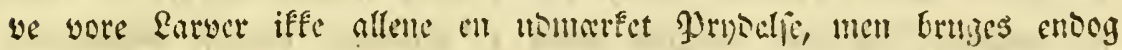

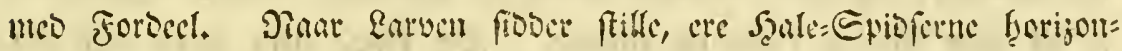
tal moftrafte, og far tat famlede, at be fintes at mogitre ell ene=

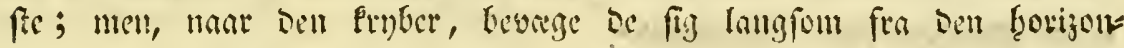
tale til ien perpenticulare Einie, Dog thell at Epret blottes; og

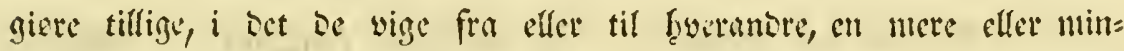

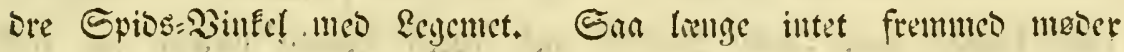

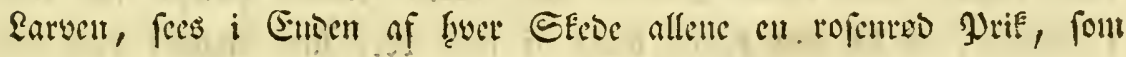
ere bet yoerfere of Eproct, men reter fiende bet mindife levenoe, enoog en af bemoes eget Elingt, fretter frum fig frat til \$arge,

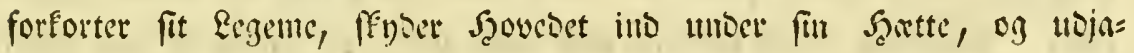

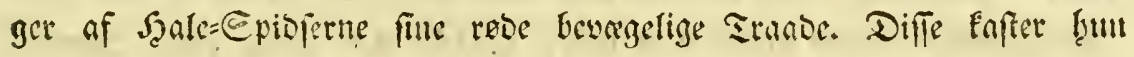
frell over Scoucset, eller beyer. Dem i twende Epitaler mod fyeran= Dre, og veo Mige 3 endinger fordriger en formodet frimbs. Siogen

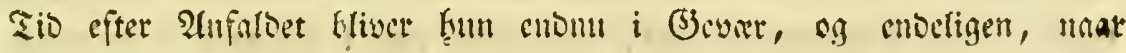

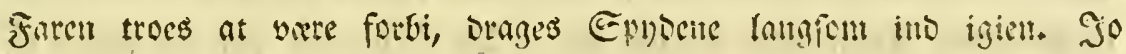

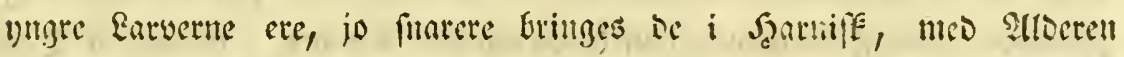

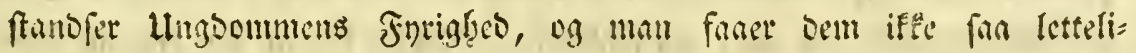

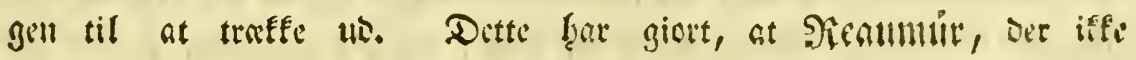

$$
\text { D } 3 \text { bar }
$$




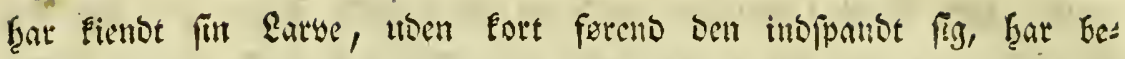
fFrevet og afteguct ben iffun at bruge et Epro, fon byan Ealoet

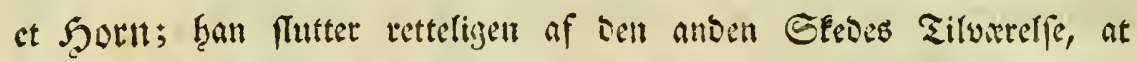
ben maa inbeholde bet altoet. Daar jeg f̧ar vilbet have bum fra

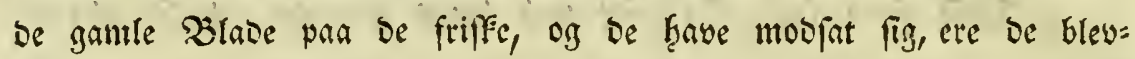
ne opfragte, og Grave bevaget beres $\mathfrak{i}$ Epiraler bryede Epyde vel ti til toly Bange frem og tifbage. De flende bem mere eller mins

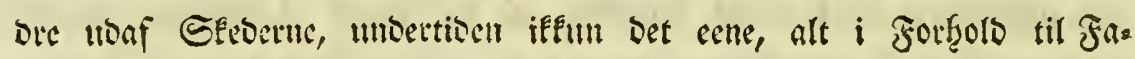
rens Storfed eller 3eovarentoe.

Branot Den Manngoe, foul InEfeligen fremeom of Zeggene;

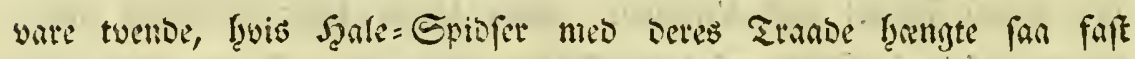

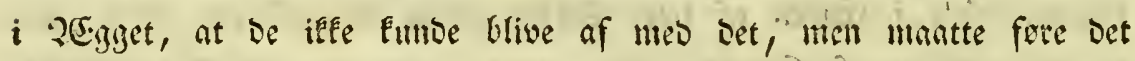
med fig, ligefon en Enegl fit Scutrs; man vilse of have antaget bem for en faaball, fyuis be ware feete treen for be andres Earts

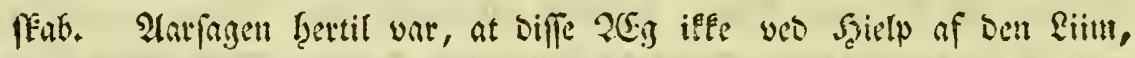

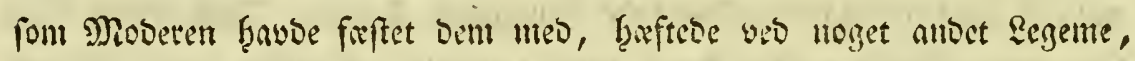

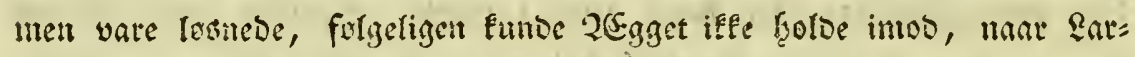

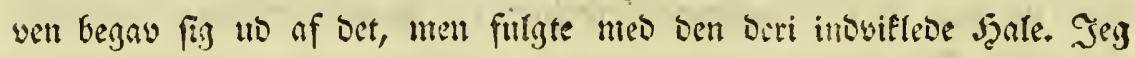
Sentroe med Begierligheo paa, fivorlebes siffe $i$ Mangel af at fume butuge beres 3arge vilbe begaae fig. De frebe med lige Syutighed og

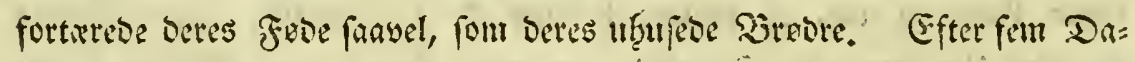

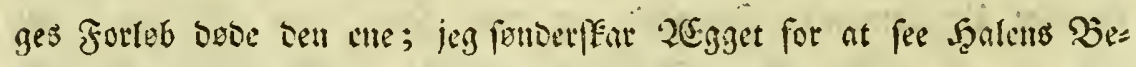

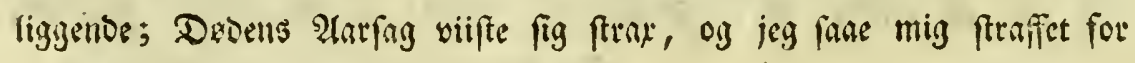
min 


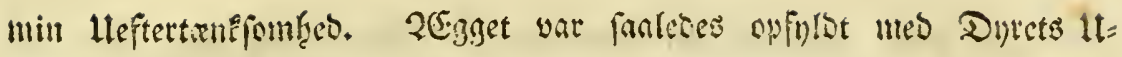

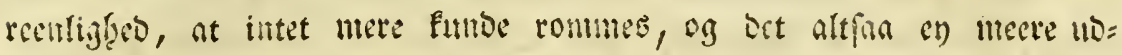
tomme fig. Seg tog freap fat par Den alloen, for at beftie ben fira fit en mere nuttige $2 e_{3}$; Det fintes og fou Rarvelt wed fivert

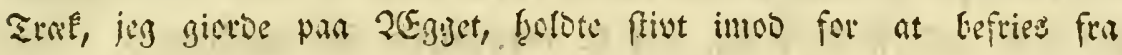

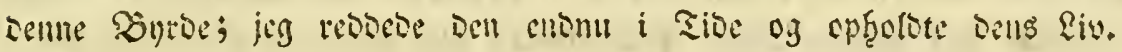

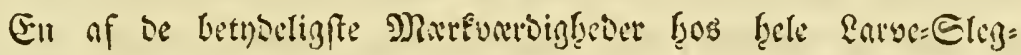
ten er Deres tnderize Subfififtelfe. Det er hos sem, Slangerne,

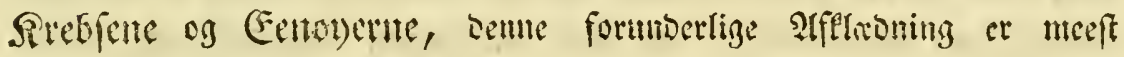

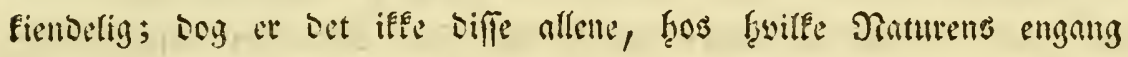

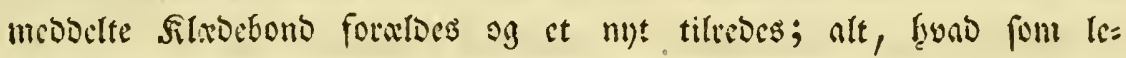
ver, er mere eller mintre unoerfaftet Denne Swon. Şvem er bet uthes

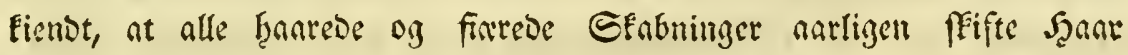

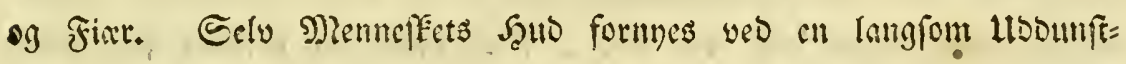
ning og Evede=Treviernes umerfelige Afpragelie. Szuorledes ellers

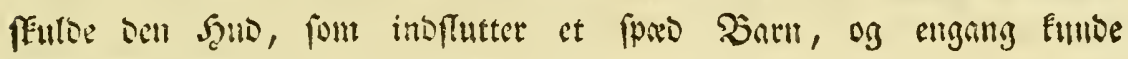
paffe til sil fulbuoken Sinkl.

Da jeg ben tectie Sunii efter Erroune vilte give mine Birs fier fripte Slade, bley jeg vaer, at nogle fijt og her yare tegnte= se med Rarmefintrebe Marker; jeg Muttede fmart, at bet war en Birkning of benue forefanenoe forandring, ag at fruben par be

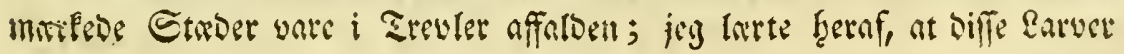




\section{2}

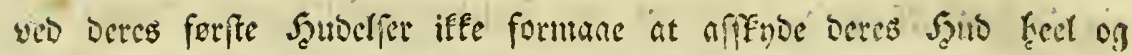

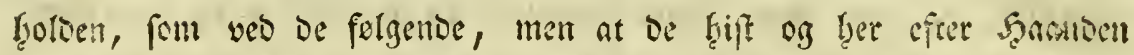
man afgnave Den meo Deres Trmber. En brog i far min Dpmarefon=

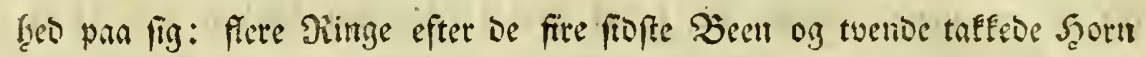

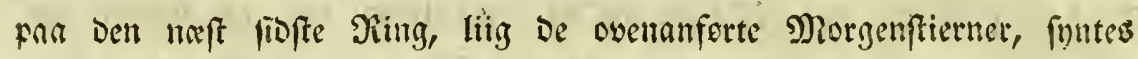

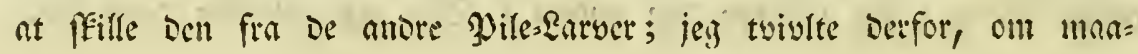

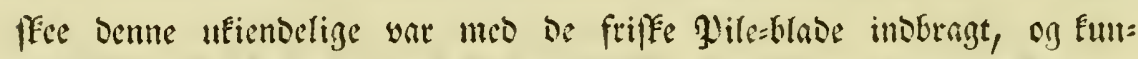

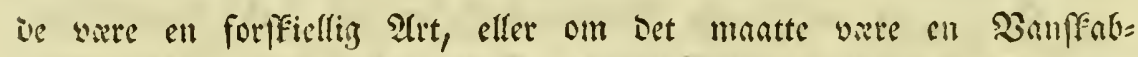
ning, fom of en Sarnbelie var Gleven bifat med Diffe twende Bes warter; Beri figrectes jeg veo bet, at Earweri nogle bange bevede

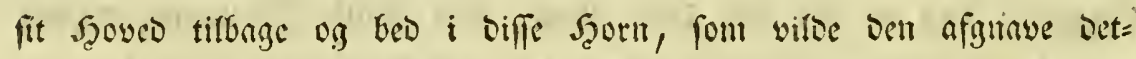
te ubeckylige og umebvendige. Smislertio forvarede jeg ben for fig allene for at fomme efter Sandfecon, og Hev chomu fanme Dag cuerbevifif om ben $i$ Taturlaren faa nlmineelige Satnings Yipper. lighteo: man ma lebe og finde, iffe tanfe og tilfutte fig, fiono Natuten givr.

- Seg bley ved at opmerte miure Enrver, og fane, f̧vorlibes bet

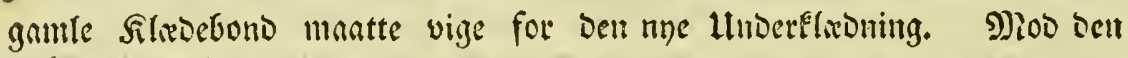

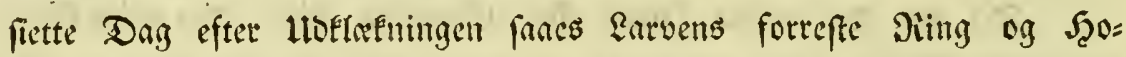
ved at opfuolme og blive ferere, og froved= SEallen efterhanden at

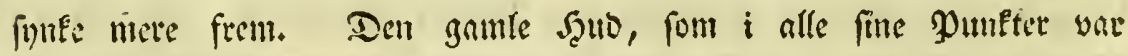

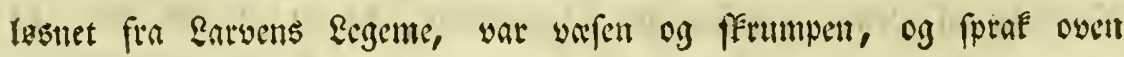




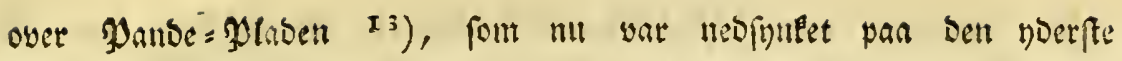

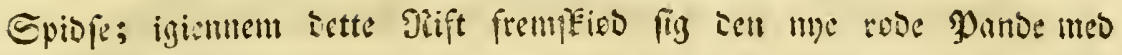

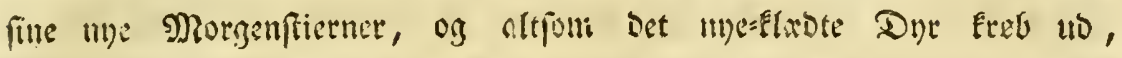

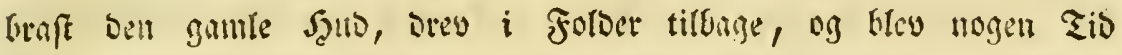

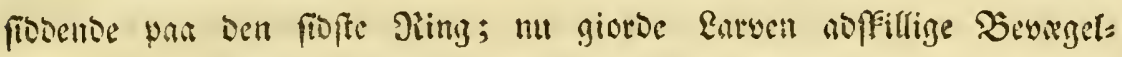

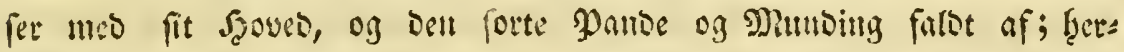

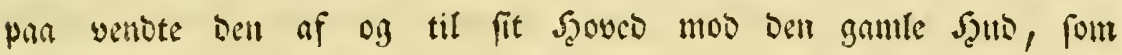

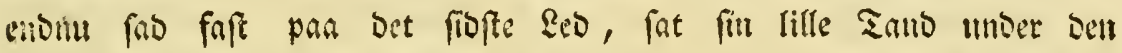

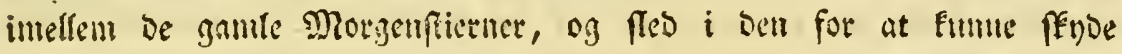
Den ouct Eproene, four fuart paafulgte. Errak faacs Rarwen at yare blenen feere, $i$ fer Dens baarbere Deele, fom war Den fande

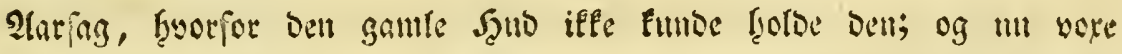

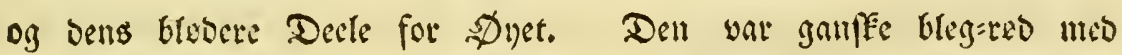

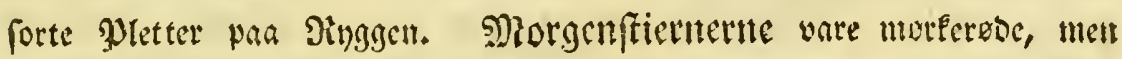
bleve fmart forti. Spybente eller be robe Traabe fintes $i$ et, ment

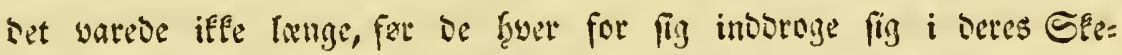
ber, og biffe fie seres fima Poigger, og fanes omginue med trende

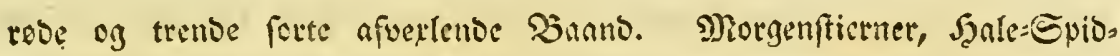
ree og Sttmper af ben aflagie Şut laae omfretete, og abyatede iffe at tage en alts tiffortige forantoringer for forpfirallige.

Earve:

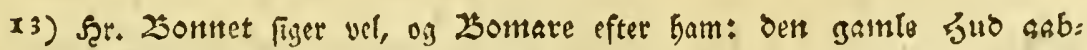

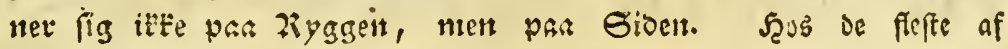

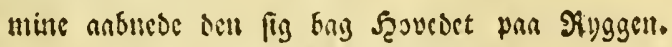

E 


\section{4}

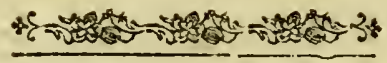

Rarve= Siembere ${ }^{14}$ ) have vel foregivet, at en Rarve ved fin

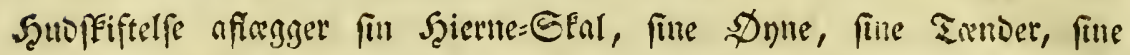
Been, med et Sold alle fine ubvortes Deele; Dog forlyoloer set fig iffe egentligen fralcoes; Den affleter fig veo fine Srublfiftelfer alles ne Det ene Svertraf efter bet andet; man feer tndeligen, at Den af:

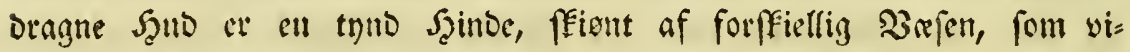
fer Eporet og Smbtrnffet af alle Earvens movortes Iecle, og iffe

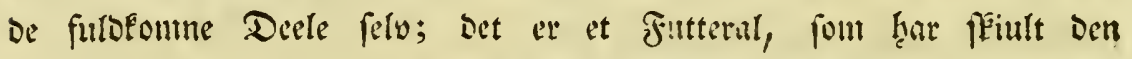
nuefobte Earve og nu med alle til be Howortes Dele paffende Sino: triv) ert aftagt.

Bel fees i Sammen Şierne=Sfallen med fit Tand=-Jiarbe og

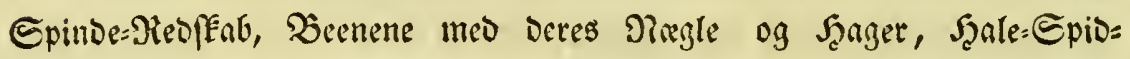
ferne med Deres Pigger og fros ie Shaar=rige en Mannge f̧aar af forffeillig Slags, men alle bilfe Dele ere Glotte Sucrttrê, Der ţave indofluttet andre, fom nut cre Glevne fterre og have forbevet fine; $i$ beres Fum forbrives be atter of anore, og Dette vebuaret faales

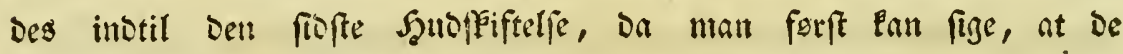
fullofomme Dele aflegges, De nemlig, fom allene f̧ave Earve=Standeur til Donemes.

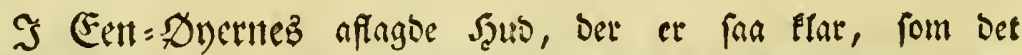
tenfite Siriftal, fees bet mindfte Scanr, or minofte Side=Stranler i Firrene

14) Bomnet confiderat, fur les corps organ. tom. 2. P. 175. Lyonet traité anat. de la chenille, qui ronge le bois de faule, p. 7 . 


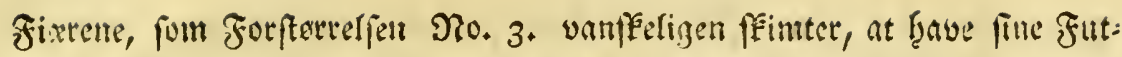
teraler; Gyilfen Eidenbed, fom fratt overfiger Decmefects begreb,

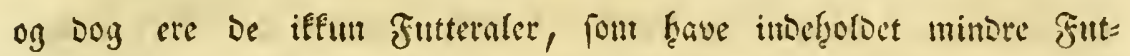
tetaler, ber indeluffe enomu mitrore levente Drganter; endog fanoan= ne Decle, foum man $i$ bat fullofomue Dyr ifle fan blive vacr, op:

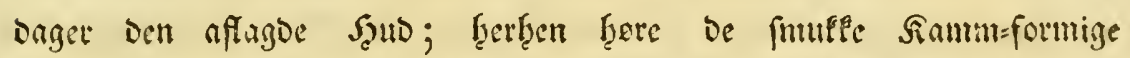
Eunger, foun tilligemed andre indvortes Deele vedhange Eenrye= Szammen.

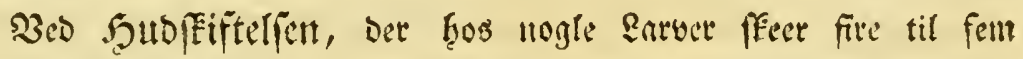
Bange, fros anbie otte til ni Bange, gaaer bet Diffe Sulfefter, fom de arrore Dyr, naur be farbe: De blive fuage, og wo intet, faa lenge bette Atrbende panfaner; ocres Farve foranorer fig, og De frave iffe mere Etyufe i 3 chene; vil man pan ben Tio tage og

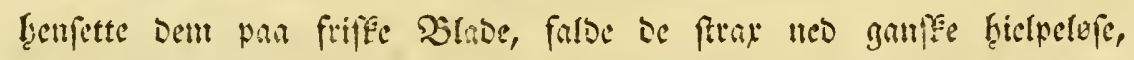
Da be ellers fefte fir fara faft, at man iffe usen meo Magt Ean

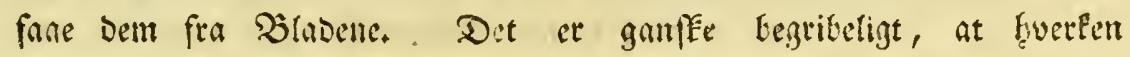
Den rette Farve clice nogen Siraft og Saxftelfe fan mere vare $i$ bet

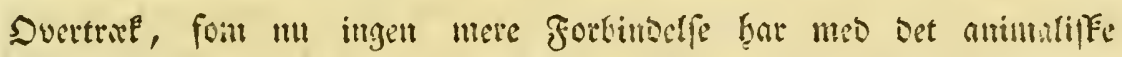

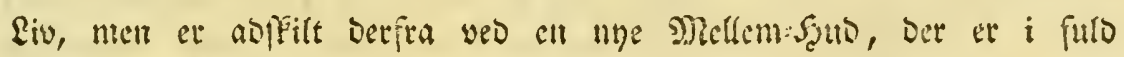
Sirnft og Firbeyde.

Mau Runde ber faurge, fiar ba Naturen glemt fin Forjorn for biffe Selabninger, naar be meeft trange, nemlig i Bcres fuage 
Tilftand? Ciben de ifle fan faffe fig, wil be ba ifle letteligen

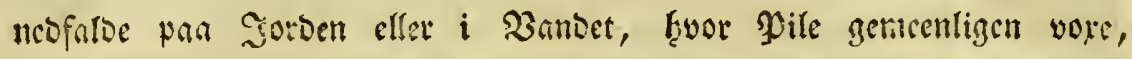

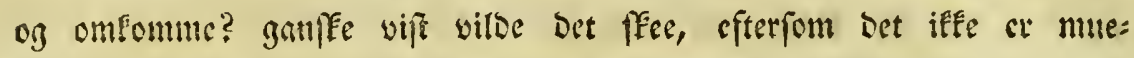
ligt, at et Drgar, four ifle spfylbes af noget Revente, fan bolbe

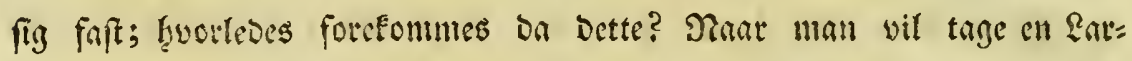
ve, fom fear fienjat fig for at peifre, fra fit Eteb, finber man ben far faft weo Brubet cller Binffet, at Den fterfe Bevalt beferwes til at frarive Den; faved er Det Da, fom froloer bon? De Sagar,

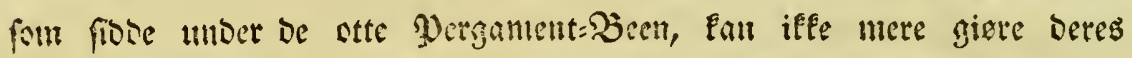
Forretning, Da be ere fFilte fra ben levende Maffe; de fer fyornag= tige ere af famme Brumo lige Erofteslofe; Dog fee Naturens Snt

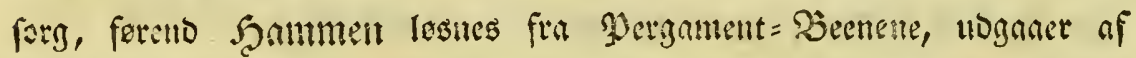
Dent an Slie, byoraf Eneven traffer facm og tilfage et Exino xs) $i$ jmaa

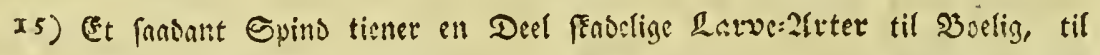

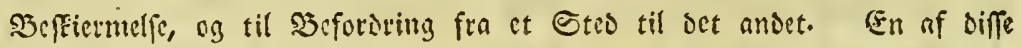

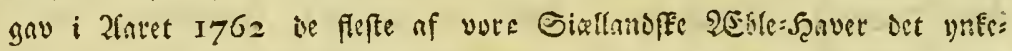

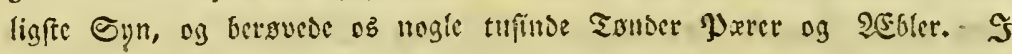

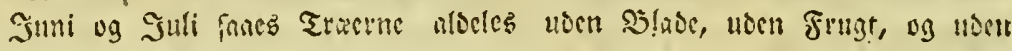

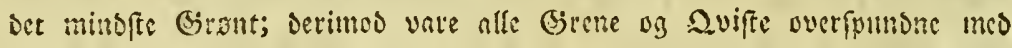

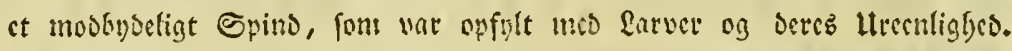

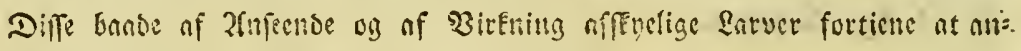

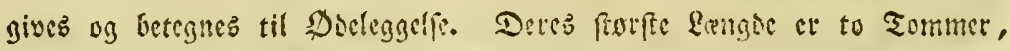
og Breich $3=4$ Rinirr. Legenet er brantgunl og glinsfende uten

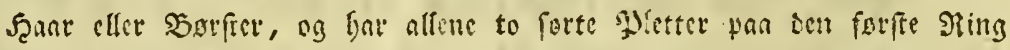

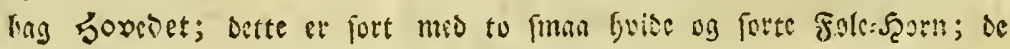




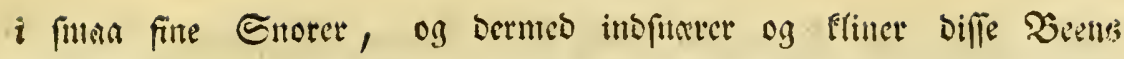

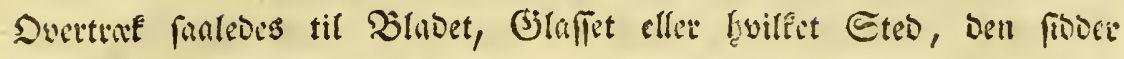
paa, at ben iffe fan fraflibes, Hoen at bette Epins forger meb;

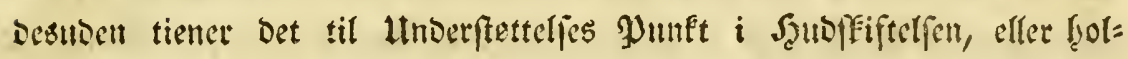

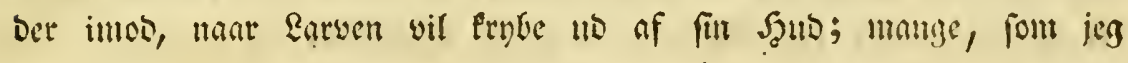
les les fra Epinoet, for at fatte bem par frifee Slabe, ba jeg

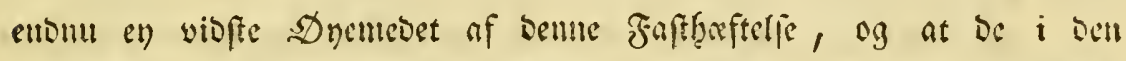

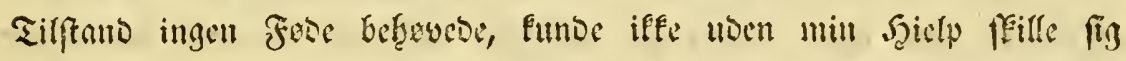
veo Beres Sam, fioen be el) formanco at fremtringe et myt Epint. Den bley finngende ved Salen, indtil jeg yeo at tage fat pan

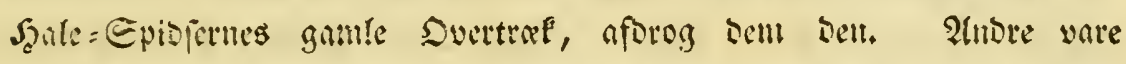
garoffe vanfungtsos og miften bobe; De funde of Mimngel paa set=

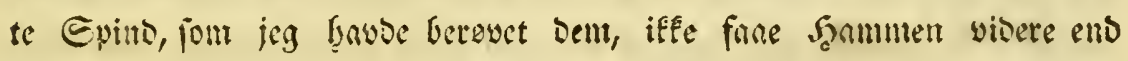

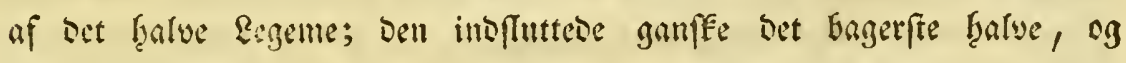

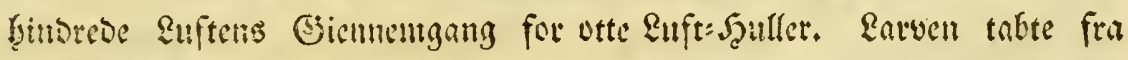
et Mimut til ancet fin Farve, fammentunfedes og vesuncos; enbeli=

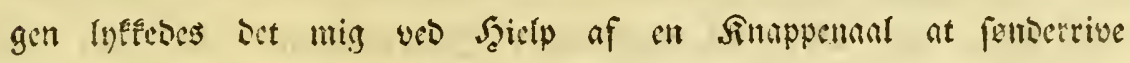
Dens gamle Silvoning, og fauledes at reose dens fiv. Sit ef= tex lit fegunte ben at roe, og at fane fin farve og Tri=

$$
\text { (5) } 3 \text { velle }
$$

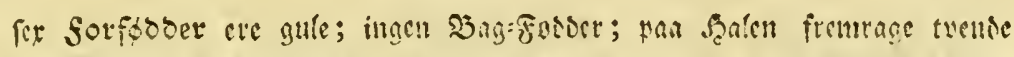
spigger. Seg tog nogle til mig for at vide derç feele seifforic; be

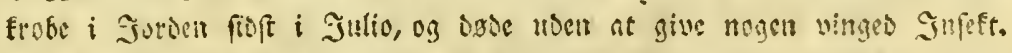

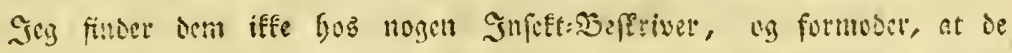

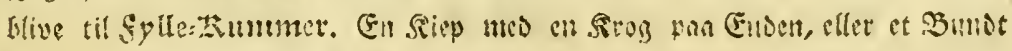

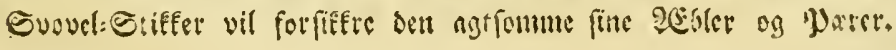


welfe igien, foum be anbre. Sit feect bet, at farven $i$ fine Shubs

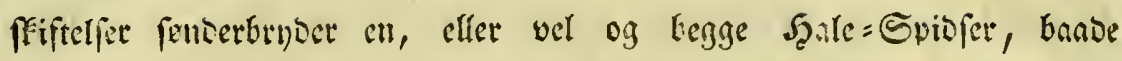
Sfede og Epyo, bog gior bet Den ingen vibere Heciligheo eno at

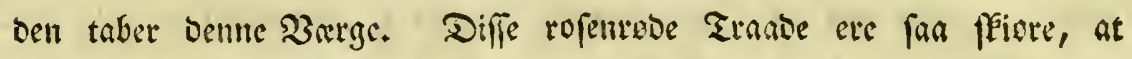
et Styefe, maar be veoreres, letteligen afbreffers.

Seg wil iffe optyoloe mig med at beffrive be igientagne f̧utbs peiftelfer, four vores Earve b̧ar tiffarles med alle Rarver; Det bliver nol at anfore bet, fom i far tilfommer benne 2fet. Sed ben tre bie eller fieroe Satholfiftelie tabes ${ }^{10}$ ) Morgenfiernerne tilligemed den

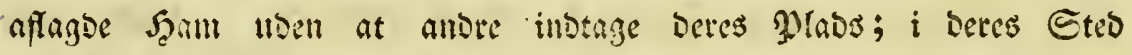
fees twenoe fore forte Pouteter, fom egentligen ere twente Sauller. J̧ubelferme ungtet beţolbe Earvens Sing fin forte farve, faa lange Panbe Shornene yebblive, men bugen og Sideme foranbe fig fra Det merl=rede $i$ bet foovel:gule. Nogle faa, fom jeg havbe ubfat paa Pile=Traer, vigede fra mine Satub=(jixfter Deri, at Singgen iffe

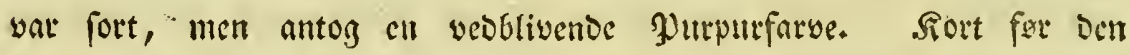
mifer Syornene, begnnoer Dinggens forte Farve at foranove fig $i$ bet brume, og at omgives med en fyuid Rams; Bugen og Siome bli:

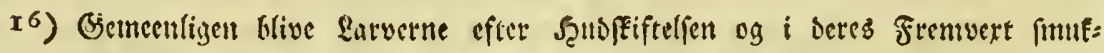
fere; jeg bar fundet nogle paa Eenen, fou ware grentre og overalt be:

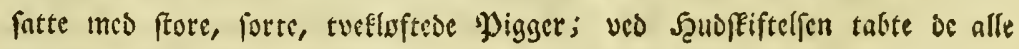
Jizget, bleve naghe og bestigi. 


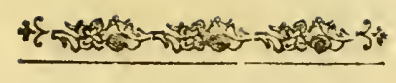

\section{9}

be gress:grenne, Eufthullerne finulige, og enoeligen fees fele Earven fullovoren faalebes:

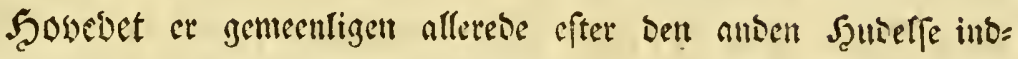

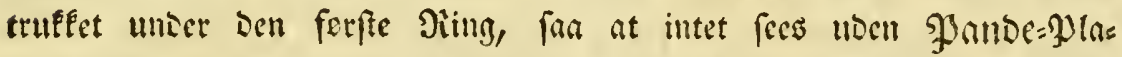
Den. Demue er fyornagtig og brun, pan Eiben fortglinsfente; oven fra neiganer mist ower Momiden en Etreg, fom beler fig i twente Brene. Secoen for siffe Hoganer fra fiver Eibe et libet gult

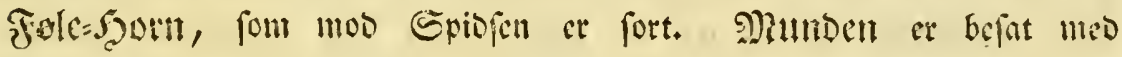

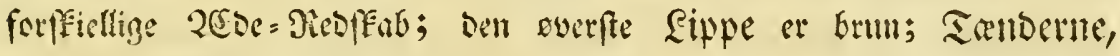
fom fra Siscrue move fyuranore, ere forte; Hoen for siffe fremras

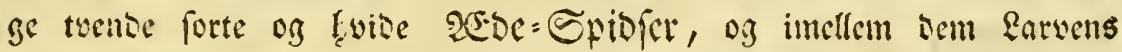

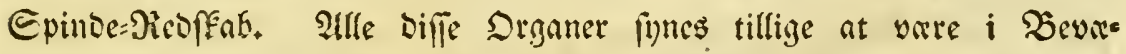
gelfe, naar garwen roer eller tugger. Waa Sivggen er Earven purpurfarbet, med fine f̧uide Etreger beftret, og fra Sioen ind=

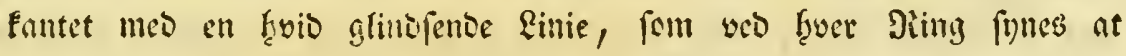
ware afbrubt. Dennes Segning fan foreftilles unber trende Triangeler: Den ferfte gaacr fra Shovedert alt fimallere op til Den trebie Pling;

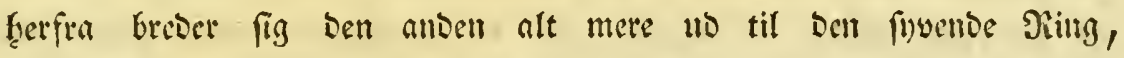

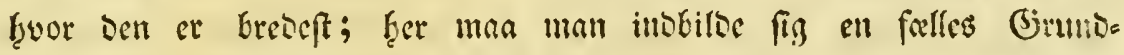

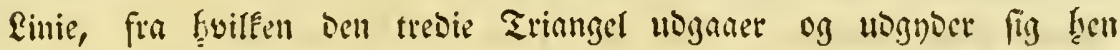
imso Santen, Dog uden at famles $i$ nogen Epioje. Siderme ere eblegrenne, brede, opfoolntede og giennemffimende, befatte nted atten oyale Eufthuller. Diffe cre hyibe, nuto ent fort Siant, 


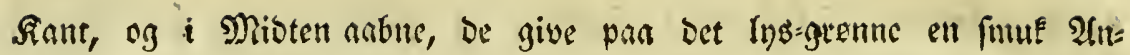

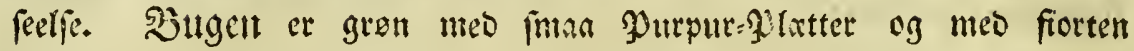
Been, fer hornagtige og otte \$ergninent:kignenoe. Diffe tvente flags been have be flefte Earver tilferlles, i frer be fer feornag=

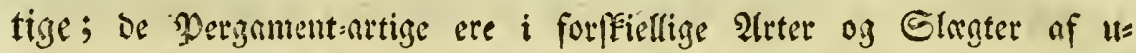

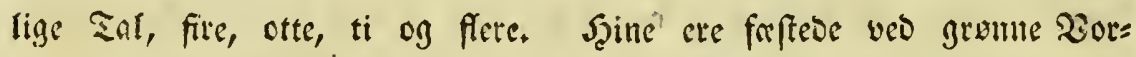
ter, ligne ell frumbeyet Enct, og beftare of tre $\mathfrak{E}_{\mathrm{co}}$, og elloes $i$ en fort fwios Sirog, fom allene formaer at bare feete Earven; paa ben indoendige Side ere be f̧uide, paa ben noventige grenne; Binmoledoet er betegnet med twende forte Pletter, Dit ander og tres

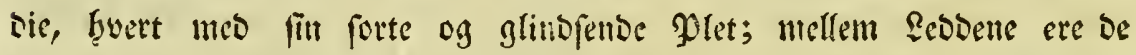
bleg=gremne, og pan ben intwentige Eibe fees chom to cller tre

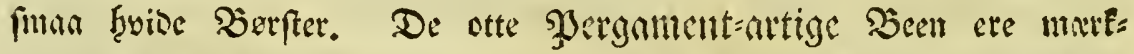

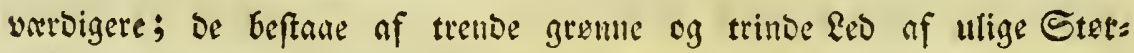
relfe. Det neft bugen er fererft, Det alrdet ar minore, og para

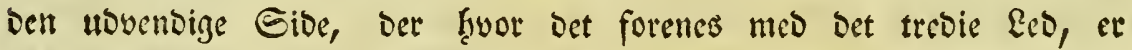
Det oungiven med en fort og glinofente rand, fon er befat mes nebţangeno grane Berfter, liig \$Dyenbrymer. Det tredie er minoft, og bar paa Entoen at Eegeme, liig en Fobjaale; scme ftaner for ved og til kegge Eiber frem, og er $i$ en fraly Cirfel intofattet af

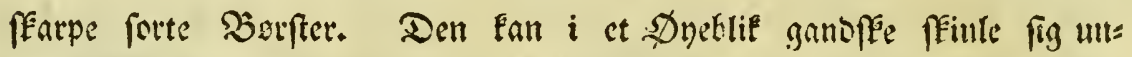
Der be forte Dovenbryuer, og atter novide og fofftlamre fin faas yel paa enfiver bjienftano, fom mod f̧uerandre, da ben ene Foos Saale gandffe naye paffer $i$ ben mobfatte. 
Raryell beftaaet af toly Ringe, foul ten ean ubvibe og ind: enite, og faalebes efter befrag formere eller forminoffe fir Enengoe.

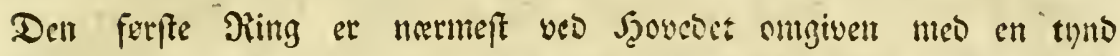

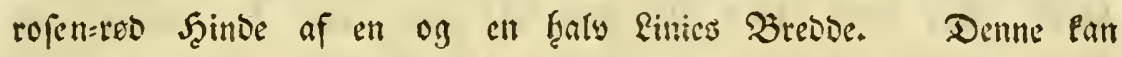
Earven ubbreoe efler indorbe, fout bell vil; bet ferffe ffect, naar

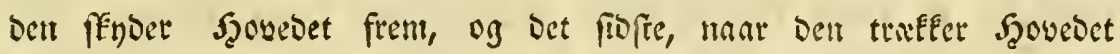

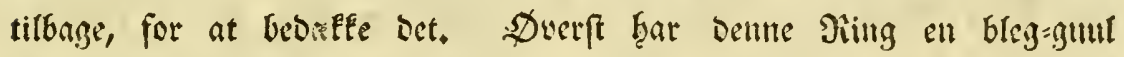

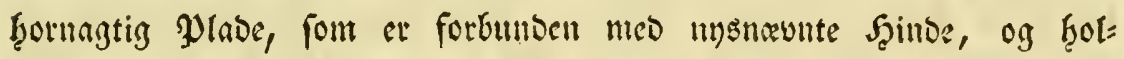

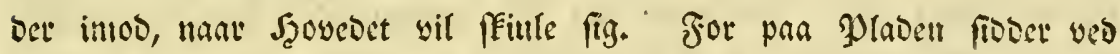
fyeer Site en fort $\mathfrak{P l e t}$, Det fintes at frave en liben Foronbelfe, og f̧ar indaget Margenftientuens Plabs. Paa Siserne inbluttes Den rabe boyclige Shinbe of en gutuleagtig Etreg; og bet ţele gi= ver bet Billeoc, fom Sienumir figer ikfun fattes en Slvife for

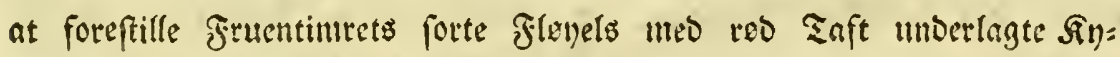

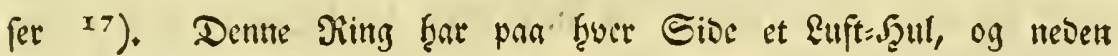
tumber

17) En ansen forfatter gior ferover en ulimagelig moralfe Setragtning og

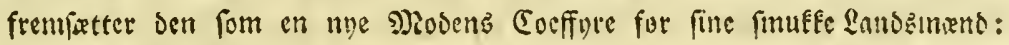
je voudrois fravoir, Clarice, ce que vous penfez de cet ornement de tête, fin vous le trouvez galant, ou noble ou bizarre, en tout cas remarquez, qu'il raffemble les couleurs de trois ages de rotre fexe \& les place judicieufement, le blanc, le couleur de rofe \& le noir, l'enfance, la jeuneffe \& l'age mur. Vous avez vu parmi nos infectes des habits d'un gout fingulier, il rous manquoit de trouver une coeffure, qui n'eut point encore fa pareille parmi nous. Hitt, abreg. Şuilfen 2 fn:

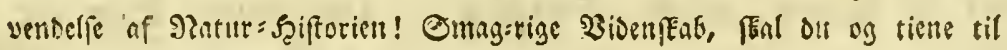
en galant Gualimatias? 


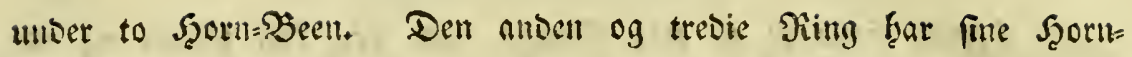

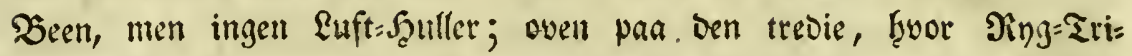
anglerne modes, fioder ell rod opheiet Siltep; den finnes at yare baaro, men foles blod; fra Shovedet gaaer over de twende ferfte

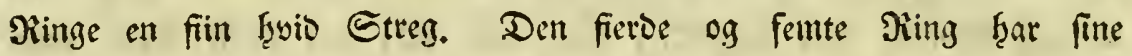
Eufthuller og under Bugen brune Wletter, men ingen Feboer. Fra ben femte Siing gaaer langs ower Singgen en blaa Sinie, fom vel bliver en giemmenteinnende Tarm. Den fiette, finsende, ottende og niende Sainz frar Euffthulfer og De ovenmelote Pergament=artige been. Desuden fees byos de flefte af Denne $\mathfrak{A}$ tet pan ben froende Jing neo mov beenette en ftor rumo Julet, af famme farve, fom Earvens Ping, Dog unbertiden noget lyjere, og ongiven mes en bleg= gutul Rans. Paa nogle lober ben $i$ et med Den purpurfarvede Tri= angel, fom beveffer Minggen, Der f̧vor Dell er bredeft. SGGlant.

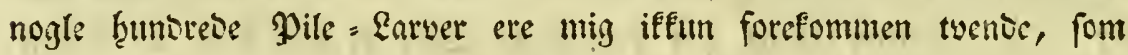

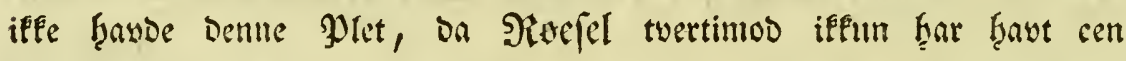
med Denne $\mathfrak{O l e t}$ og $\mathfrak{S}$ r. De Gieer nogle. Den tiende og ellevte Bing fiar og fine Euft=Şullfer og langs unoer Butgen tvende brune Etreger, fom ende fig pan ben tolvte. Friflch holder for, at bifie Etreger ere Mierter paa Şaunerne, ment b̧an hiur Htret. Mellem Stregerne fiode par den tiende-toende ftore og tuende fima olet= ter; De frore beftane funt of 2, fuart af 3 fanmenlobne Pletter; enomu pees fros nogle twente Spunfter; paa ben ellevte Ring ere il= fun to Pletter mellem Stregern. Den tolvte Ring b̧ar ingen 
Euft=SGuller, men twende Putufter under Bangen; ved Deme Pring er

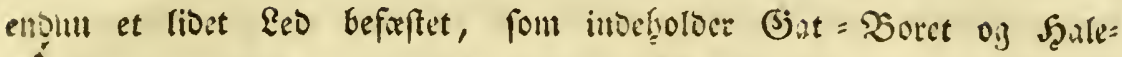
Epioferte. Diffe have vi oven til moffom betragtet, enom franet tillage at neune et par Dos om Gat=\$oret; Det beftaaer af ct Deipel og twettoe Efterfépdere. Deffelet er obal, og har paa Shanten to brede forte Striber, eg moo Enden to fina forte Snaps per. Efterfenderne, fom ligge nesen for Deffelet, ere to forte Epiofer, fom nofomme af et par granne Minfeler, og have $i$ En= sen en fpios fort Barfte. Naar "Earven vil udade fin ureenlig= Fes, briwer Den Deffelet i Baret, og Fanleves aabuer en Llogang; De twende forte Epiofer berede fig at tage imod lircenligfecten, Deres grome Muleler opjorme og brive Den fien pan Spiojerne; diffe

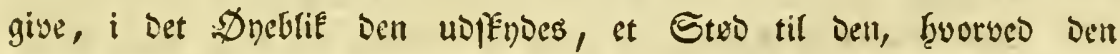

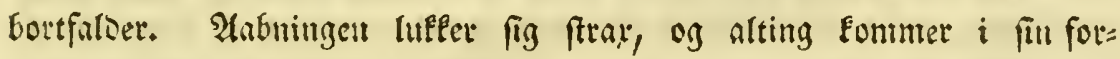

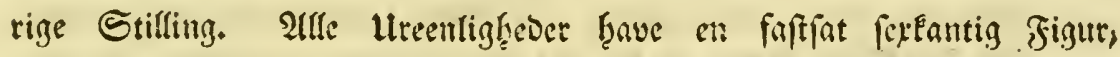
fom tiffiendergiver Ende= Snrmens indvortes Efafning, og cre to $Q_{i}$ :

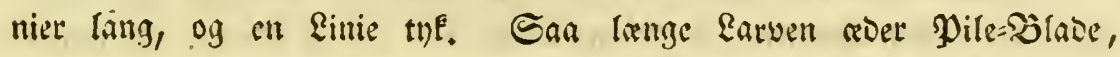

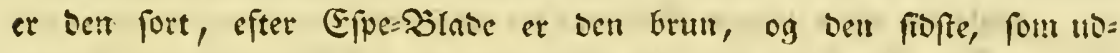
labes, naar Earven yil indpipitroe fị, thar paa den bagetfe Ende en rob Sliim.

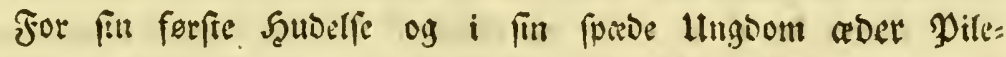
Earben, ligefom Larverne ${ }^{8}$ ) i Illmintoligţes, alleme sen grenne $\mathfrak{F} 2$

paftige

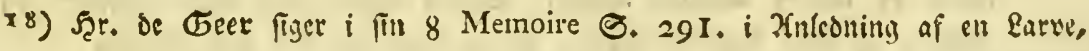
fom ader ween pan SJlanet: Man veed, at de andre Larver begynde 


\section{4}

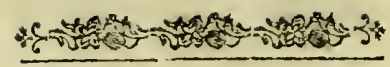

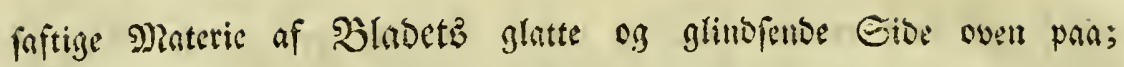

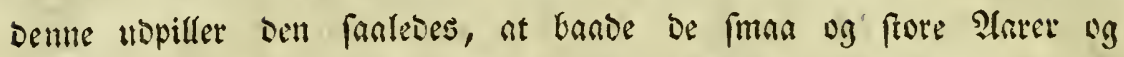

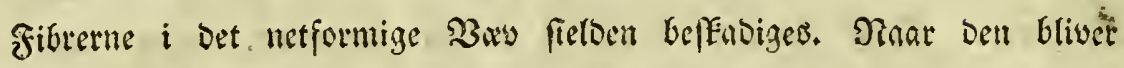
albere og frerfere, angriber ben forft be fmaa, berneft be ftore $\mathfrak{P l a}=$ ter, og enbeligen afgnaver Den fra Sinnten beele Brabet til Den

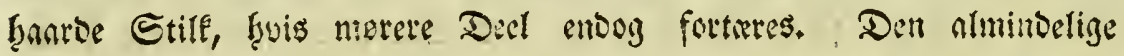
2CED $=$

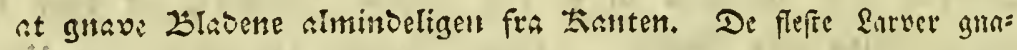
vi oyen pan Slabet, medens oe ere fmaa, builfet og beft pafiet til de=

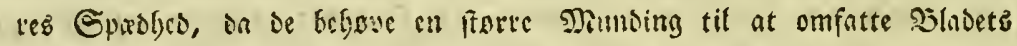
Siant, naar be. blive-ftorte og grandisere, anfalbe de Santen oy Bindets

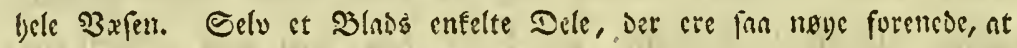

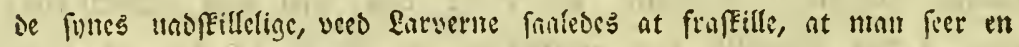
Deel at aoes af et Elagb, en anton af et andet, alle af et trebic. Mann

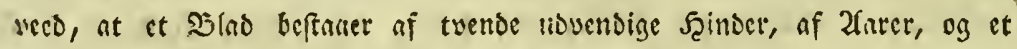

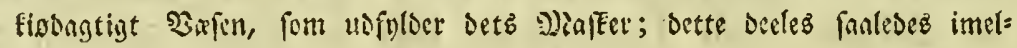
lem fire Elngs Rarver, at ben che ifie fornerner ben nuven. En 2 trt gnaver allene ben gronne oure Sinde, ch anden ben umbre, den tredic Rig: oct mellem Scinderne, og fefetere Slavet Guer pan fin Mlande. De tuente farfte ere allene $i$ bercs Salnoom fan ney) fomme, Den trebie fin bele Lar:

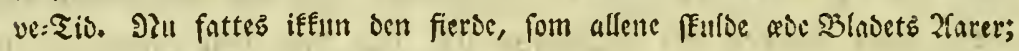

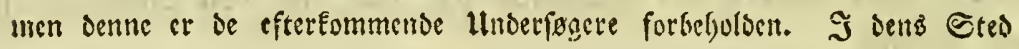

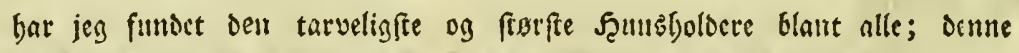

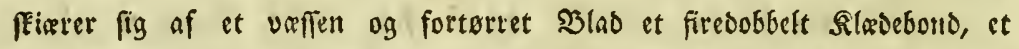

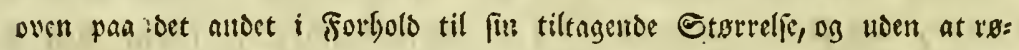

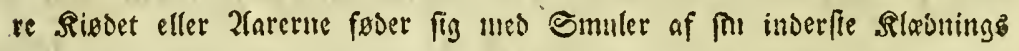
torre og meften ufintige Scinde. Entomn andre abe baade Scinden og

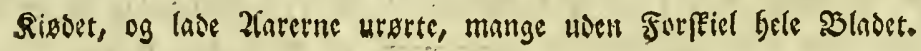




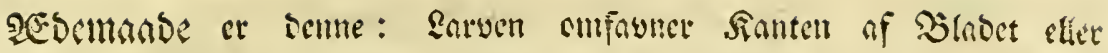

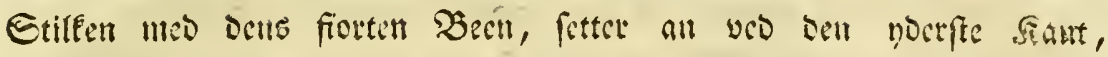

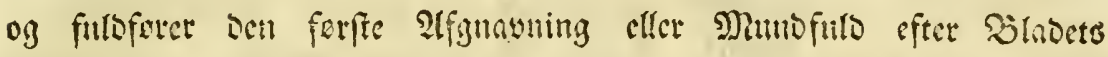

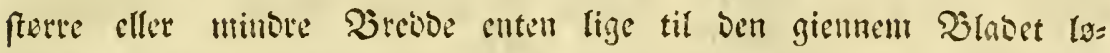

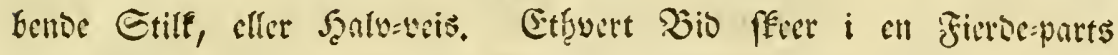
Sirfeltane af forffictlig Etartelfe. Den girt $i$ lige Tio fire til $u$

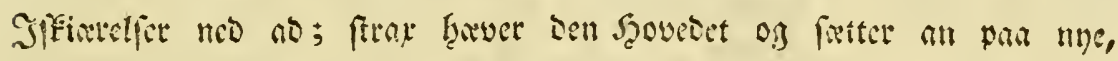

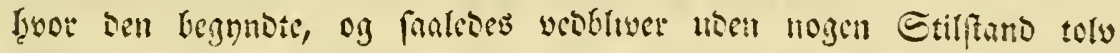
til ferten Giangs. Da 2esoningen ffect veo Suct of ulige Ete:

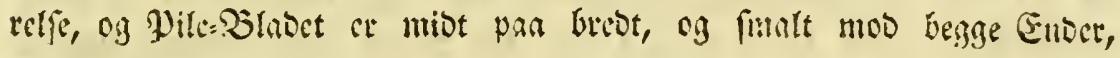

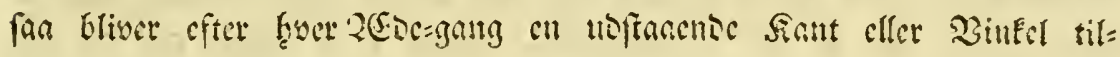

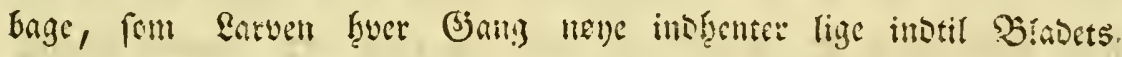
Still, i bet Den langiom vender froved og forben titbage til bet

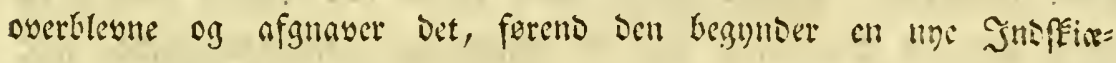
relfe fra ḃlabets אant. Er man opmirtefon, fan min ved ந̧ver

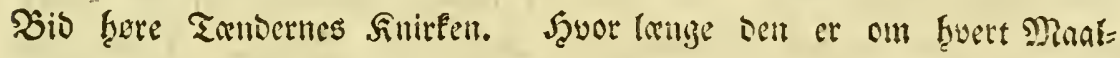
tio, og fyoor nteget Den f̧uer biang roer, beroer paa enfwers Stow relfe og Sounger; tmbertioen enocs Leoningen i fen sMinuter, og gieres en liben $\mathfrak{P a n f}$; til anden Sib verwater ben $i$ trebive til

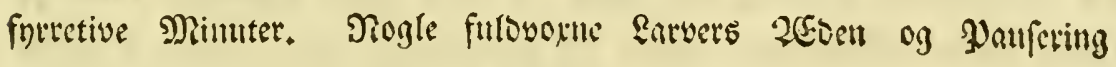
befand icg at have felgento fortholo: 


\begin{tabular}{|c|c|c|c|}
\hline 2EDen & $=$ & $=$ & \\
\hline Yaufe & $=$ & $=$ & 10 \\
\hline $2 \sqrt{2} \mathrm{en}$ & $=$ & $=$ & 5 \\
\hline yaufe & $=$ & $=$ & 5 \\
\hline $26 \mathrm{den}$ & $=$ & $=$ & 30 \\
\hline Panfe & $=$ & $=$ & 2 \\
\hline $2 \&$ & $=$ & $=$ & 20 \\
\hline paupe & $=$ & $=$ & I6 \\
\hline 5 5. & $=$ & $=$ & I0 \\
\hline
\end{tabular}

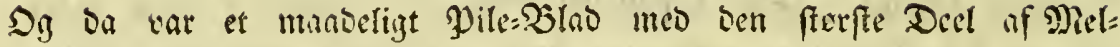
rem: Etilfen ganlfe forteret. Da jeg iffe funde vare langere marvax= retioe, lagoe jeg en af De bemartede Earver $i$ at Bjas for fig al= lene, tillige med fie $\mathfrak{P}$ iles=Slabe, fom jeg efter otte Timers forleb fant forturede. Suende Simer blive altjan den Tis, i fevilfen en

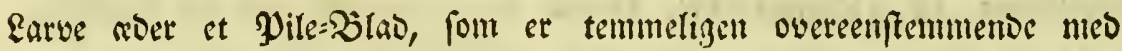
ovennuforte Bemartelfe. S falge af famme anventes af to Timer ureften halvanoen paa 26roning, og Dieften til Şbile. Denne bes ftaaer Deri, at Earven beanger fitlle Hoen at beynge fig; Dog anven= Des $i$ hyuer af Diffe Panfer at Mimut paa en anden forretning, fom jeg of Rarvens Aofiro, ter finnes at have Bette Soyemed, troer at Eunre falde Nedfufning eller Drovty Ening; Den tager Mumben fra Błavet, og bevager ben meo alle iens Jebléaber nogle Bange frem og tilfage. Den ganer ille gierne fra Det Blad, fom ben engang frar begynot at afgnave, fan laenge noget cr tiffage, men

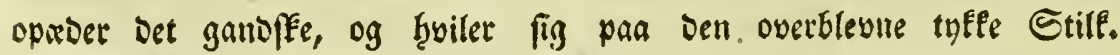




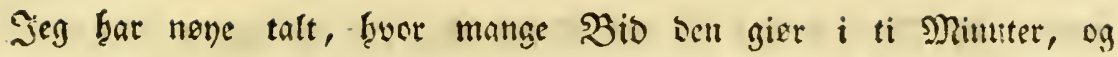

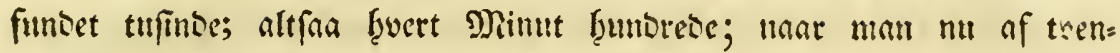
De Timer regner be fom og firfutstive Minuter par 2ersning, fa gieres 8500 Sffintelfer f̧uer to Simer. Den ereer ligefon mange altore Earver, fravel on Dagen, fou om Natten, og fioser iffe,

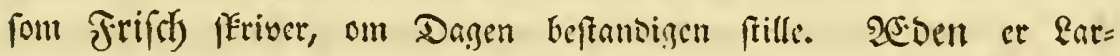

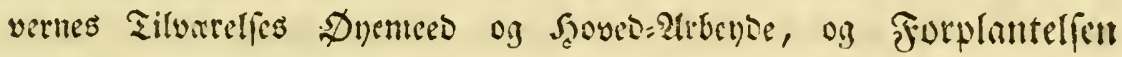
Eommerfuglenes, og med biin hafter be alt, fwoso be foul, for sit opnane Denne. De flefte Earvesatter have seres egne Ylanter, fom

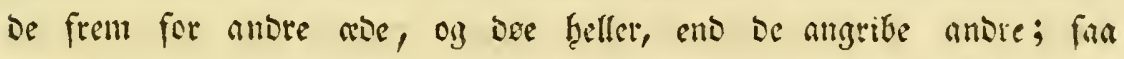
noe nange Elags; nogle ie nerbeflegtede, naar Sytmgeren neber

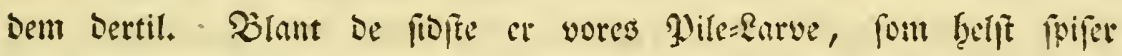

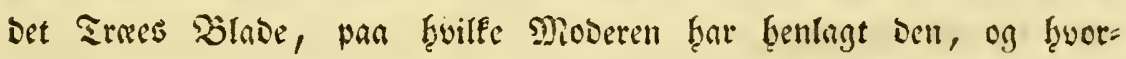
til Den er yant, bog $i$ Nangel of famme ogfia forturer alle an=

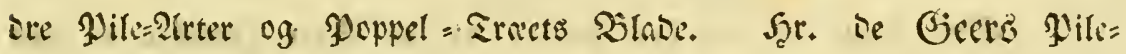

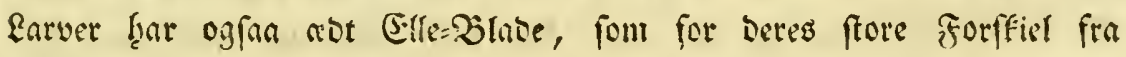

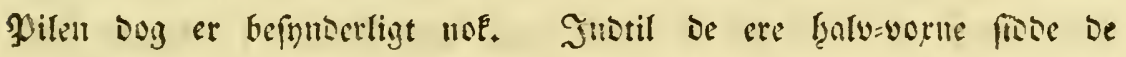
i en Eem egen Etilling oven paa Blavet, fisen baenge or gement= figen enten ved Sianten eller Etilfen af Şabene.

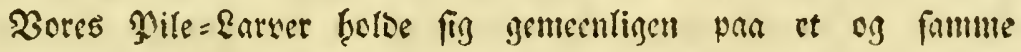
Etro, faa Innge be ber forefinde nogen llnberţulbning, eg vige ifle gierne fra et $\mathfrak{B l a d}$, fereno Det er ganffe furtertet, ia is angribe Dit narmefte; Denne Eeenorigtighed Eonmer ifke, fom Sieefel. 


\section{8}

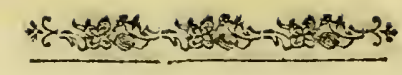

mener, af Mangel pan et par Şagbeen, fout be albre Earter has=

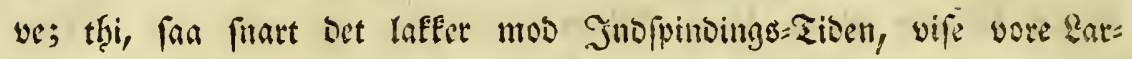
ver tybeligen, at be uben Bagbeen fan lobe meo en uventelig Scut= tighes for at foge fig et bequent Etto til Snofpitroning, on ofte

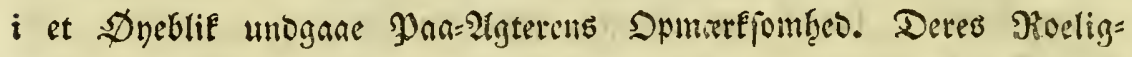
beo er sem naturlig; oe labe fig tage, flappe og frandtere far lnut ge man vil, naar be forft een eller to Bange have erfaret, at bet iffe praber bem. De beifte fig ferap til sen ferfte bent befte $\mathfrak{p l a b s}$ man fetter bem paa til beres Frose. I beres buang fette be bet trevie Yar af Sern=Bechene ferft frem, Derneft det moet og bet

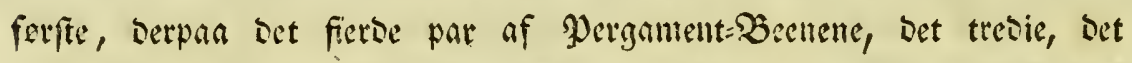

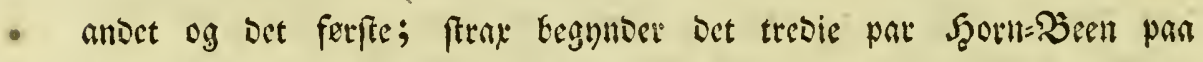
wile, og fá frembeles.

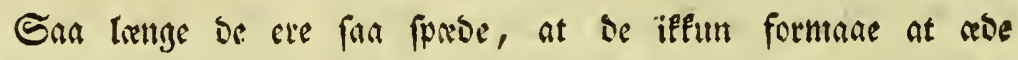
fivoet af $\mathfrak{B}$ habene, og en enomu fan thefe fibrerne, feer mant bem

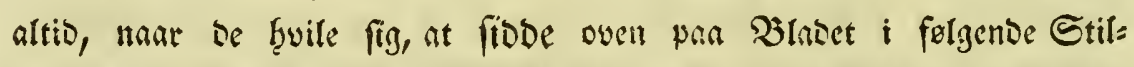
ling: De fex eller otte Peigaments= \$ien cre feftebe paa Blabets Suetflade; Den forrefte Deel af Siroppen med bens fex Shorn=ßeen cre reifte i 3 aret, og giar mes ben borige firop en ret eller frump Binfer. Ere be Gleone faa vorne, at be fan fortare Slabets 2 las ret og en Deet of Stiffen, Da blive De hrengente "eller frosento

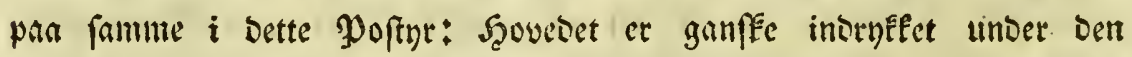

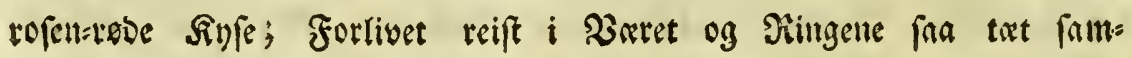

mett= 
menfnebne, at Enrven foncs en trebie Deel fortere og tulfere ello naar ben er Hoftraft; Dergaments=2Jenene ere mes Ganlens fFarpe Burfter frefted paa Siben of en tyffere Stilf; paa ell fmallere,

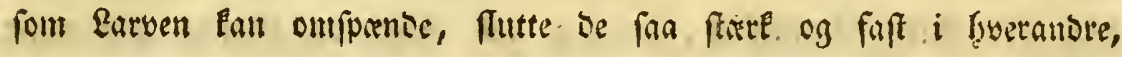
at be iffe uben 30 ban frobrages; eller og be trente ferfte Rills ge giore mes be folgentse fer en ligevinflet Triangel veo ben rebe

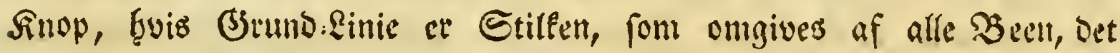

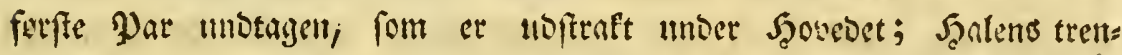

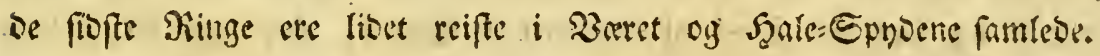

Solunt bet bejunberlige, foun findes f̧os S̈nfefterne, ex ben

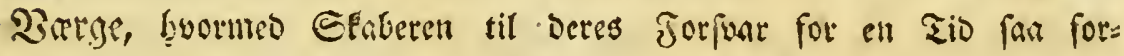

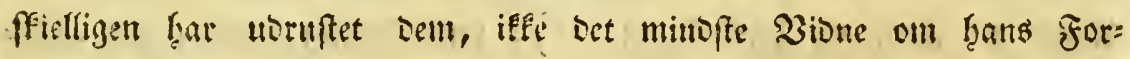
forg. Bi fitnde Dur, ber mes en Sangc; en StunE, en EvD

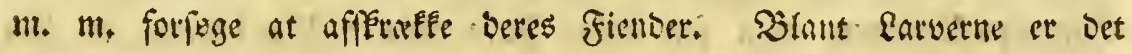
meget alminocligt at vare bejat meo Sorne of ableiflin Damelfe, meo lange og forte Sant far ftive fom Sorfter; at vare beoxfEet med en fin Duun, en SEHm, Deres egen Ireenligbed, eller at

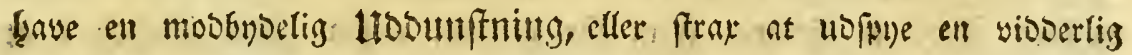

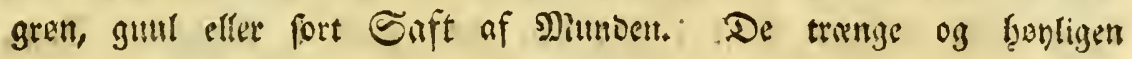

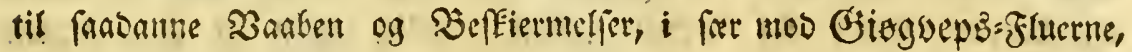

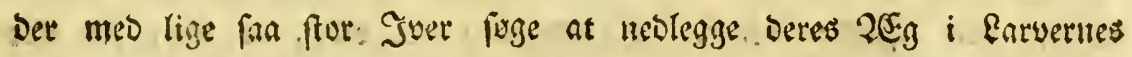
Regeme, fon Bremfen i Khent: Drtets Sigg, og. f̧vis Unger fanle Des. Mbebe beres Bert, at bean fetter Rivet til. Bores Mile=Earve, 


\section{0}

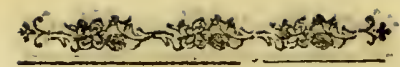

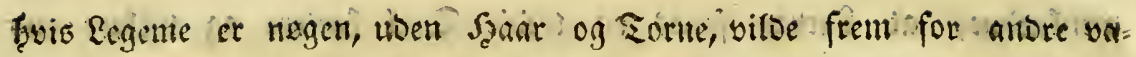
re usfat diffe Eimlte: (Sieffer, byvis ifte ben alt Eejergende Natur Gavie forfinct ben med be befferene frale: Spyo; med Diffe, foum

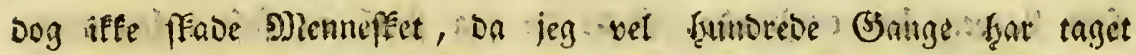
imod deres Elag, bafeer beri en allene alt, huad fom thermer fig til sen, men fetter fig enoog $i$ et Frygt inojagente poftire, $i$

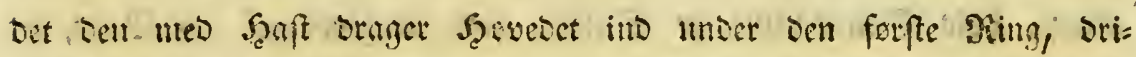
wet Forlivet beftig feem mod Den atrgribentoc, opblefer og herwer Den

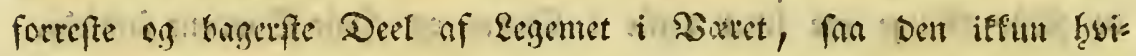
ler par be otte Yergaments=03en, og fortriver i Denne Stilimg, ind= til faren er forbi. Dog blive be iffe alle frie for "Diffe thyofige

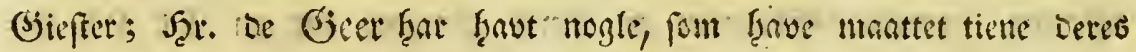

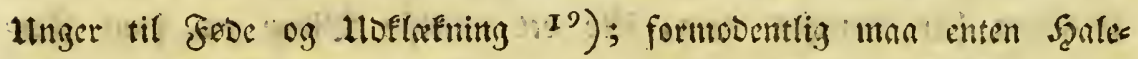

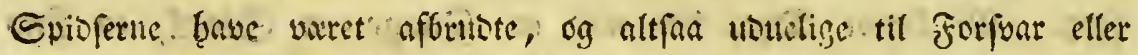

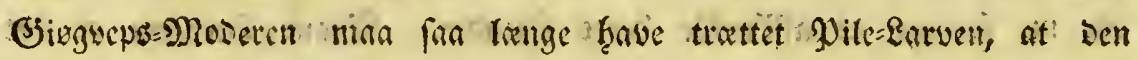
tilfoft fint varet minore paasagtende; og givet fig et 'Dyebliß blot for henoes 2ês = Eregning. Nyere er famme beremmelige Jnfelt= Rienteres Bcuntedfe, at be ursvife Ding, foum give viffe Deib

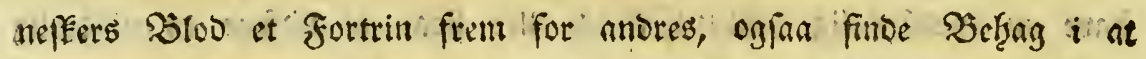
Brage biffe Sarvers Saft $i$ fig; Den fees, gren $i$ Pinggens $\mathfrak{B}$ ug,

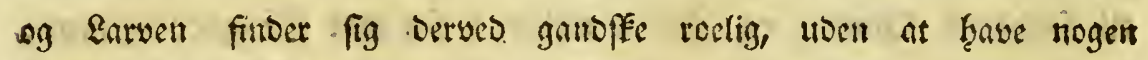
Efabe eller Jilloer Dernf: Sfulte Den vel vibe, at man for itls

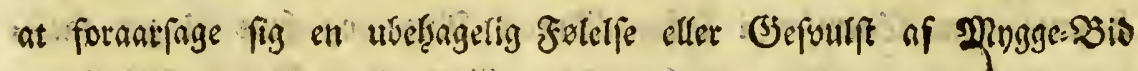
max

19) Memoires fur les infettes, tom. $x$. p. 332. t. 23, f. 17 . 


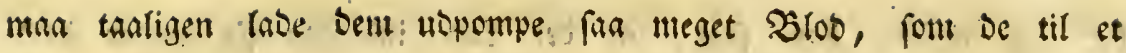
Maaltio beţeve,

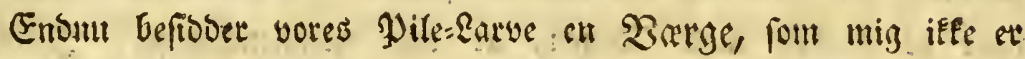
forefommen fros nogen anden, og. fou gier ben frenft marfyartbig. Denue forfeiler iffe fun- Birfning, men gier, at enţuer, ber iffe er

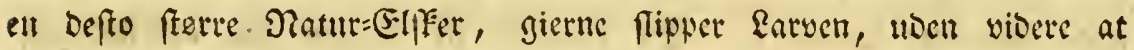
befatte fig med Den. Dette Forfuar yttrer fig fra fire Eteder;

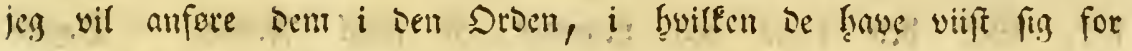

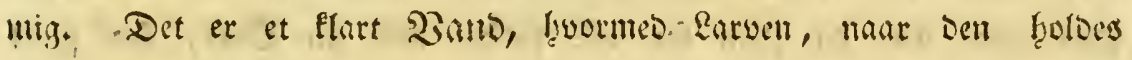

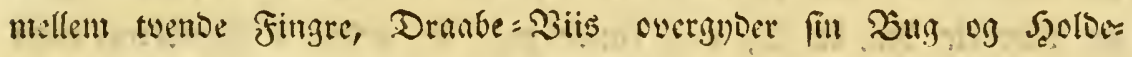
reng Saant. De ganjefe linge wolade Den iffe, fan libet fout be

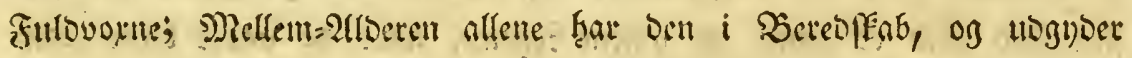
Denl far Gaptig, at - maar man troer at frave Den trinbe og tare

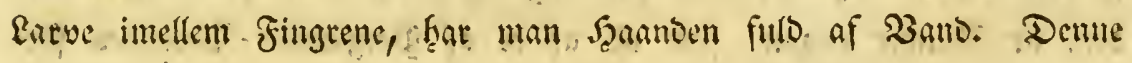
Sapaftighed giorbe, at jig nogen Tio iffe fumbe bemerle Eteret,

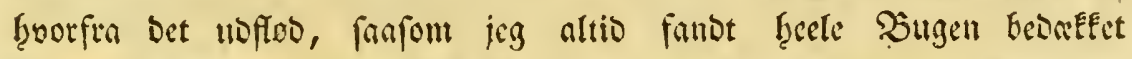

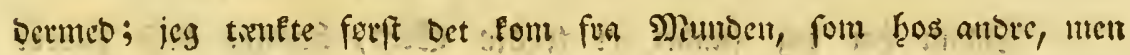
blev : fnart overbevitif, at bet mante fomme fra bugen mellem benene; jeg bles yed at foge efter $\mathfrak{A}$ abningen, men faltot ingent; elt: Deligen blev jeg vaer, at Banbet fom fra bet ene af be formefte

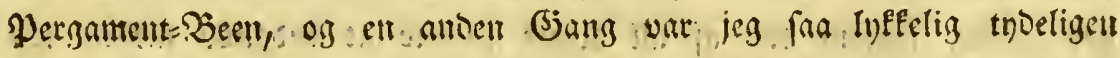
at, fee, Gquorlebes. Drauberme en efter Den anden ubpompedes fra et

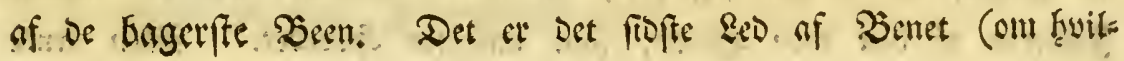




\section{2}

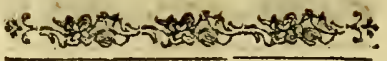

Eet jeg tilforn that fagt, at bet ean inslegge fig unber sets Doyentshar) fom Eniber fig ind og Derpa ublaber ell ftor og flar Drabe; jeg bar talt fra otte til fiorten. Selv diffe been fees, naar be frobses moo Dangen, at ware gientiemfigtige. Jgientagne

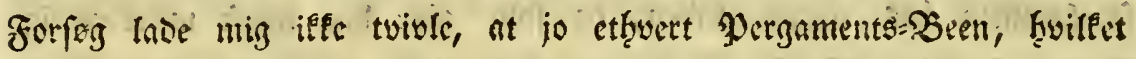
fom nermeft rerer wed roget formeent fientigt, er $i$ Stano at ub= gyde faabunne Drinter. En oyergoo fit Iegeme fan oyerfledigen, at ben efter trenbe Simers forleb enonn var vans. Saften efter=

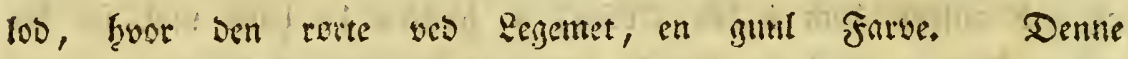
Dile= sarve war og frem for alle farbig med fin Saft, faa frart

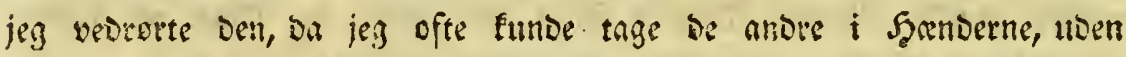
at formane sem til llogyoning; og maatti ben giengielde fin Sil= finbighes mo et gunlt og verent Regeme, haar be anbre bleve gren: he og trinoe. 'Shos farmune finae jeg og bet, foul hos ingen an=

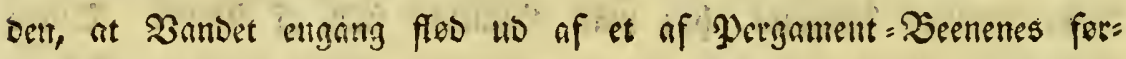
fie Reds inievife Side.

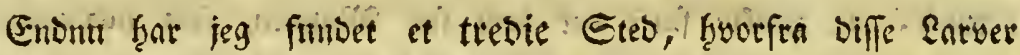
formaae at ublabe en Elar 3abffe: nam jeg hat vildet neye bes tragte Deres Manto for at abffille be Nebfferber, foni be fiar tils

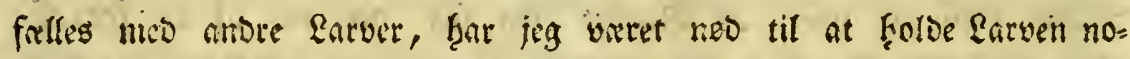

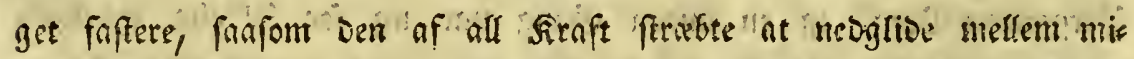

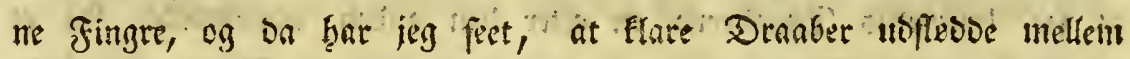

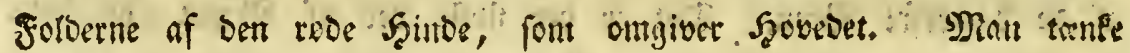




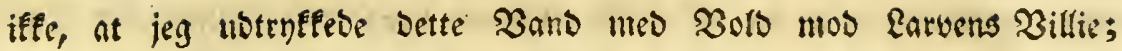
ney, bet var lige faa. fuldofommen en frivillin Sganding til Dens Forivar, fom at den til famme Tio og $i$ lige Dyemes, da Dette iffe afferrffese mig og bevirfede Dens eeslabelfe, loo et grant Sfum flyoe of fin Muno, f̧uilfet fin alminoeligt, fom bet er bos be all= Dre Earve=2trter, jeg ellers ifle bar bemmetet fros benne." Det fiet:

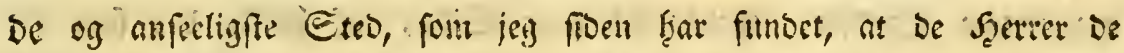

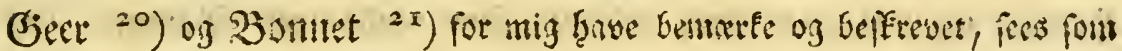

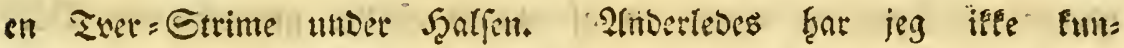
Det bemurfe Det, i f̧uor megen llutage jeg ves mange Exem= plarer frat giort mig for veo Şillp of Supen at neye fiende Dette Gteos Befeuffenfleo; Dog ex jeg of Diffe fortreftige Jils feft:Situberes Devagtighes, og of be fire Stranler, fon jeg ofte bat feet $i$ en lig affano paa engang at ubjprontes fan fulofent

20) Memoires de Mathematique \& de Phyfique prefentés à l'academie d's

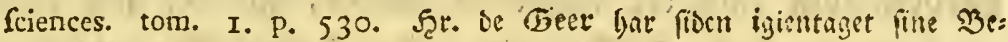
matfelfer og ladet bem aftegne i fute Memoires pour fervir a l'hiftoire des infectes, tom. I. pag. 325. t. 23. f. 7. 8. 9. Digage Sirffer bave yaret mig vanfelige at. Fofommi ;et fibfe fit jeg, in mine

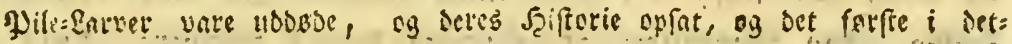
te Irri; ben altoen Diel of lyans Memoires, fom far viot jeg

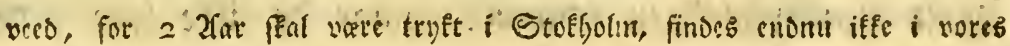
Şovid:etad; fax. fitoigen fecs elloog et Taboe:Riges Strifter, ori dog fortine all vorcs Dpmatfiomber.

2r) St de famme Memoires de Mathemat, \& de Phyfique tom. 2. p. 275. 


\section{4}

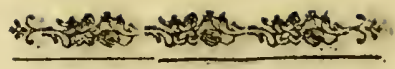

men, cucreveviift om bet af Dem anziste fire-fpibfede Reofeabs Tit=

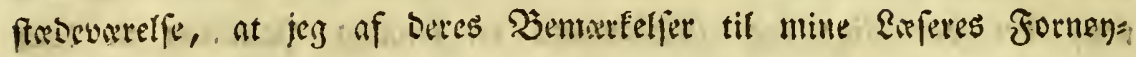
elfe, laaner Dutte Sorgang: Beffrivelfe og hosfonede Tegning. Det er et bloot og firoagtigt fegeme, fom Earven undertioen; naar Ders

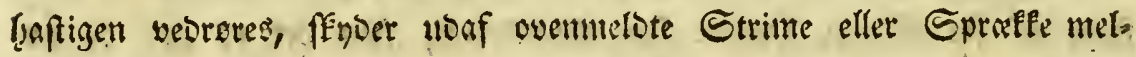
lem Showebet og Det forfte par Scorn= Been. Det beftaner af fire borizontale Epiofer, twende til Gyer Eibe, of Guilfe oc, fom ere

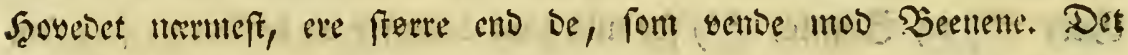

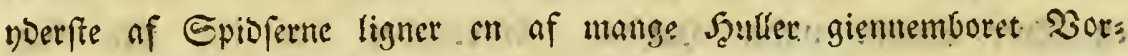

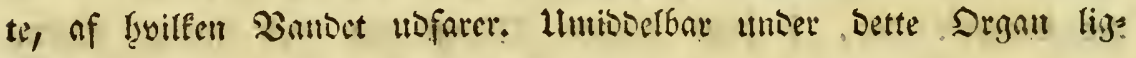
gev en Blare af en goo Ertes Eterrelje, i f̧uilfen Dandet fam= les og til panlonmmende Tilfarbe bevares.

Efter Şr. De Ģeer fEal Earvell iffe funme ubipgente Dette Bano, meo mindere ben lever $i$ fit Frifged; jeg bar fundet; figer han S. 325. at Dette befunderlige Syn ifEut bavez bo: de

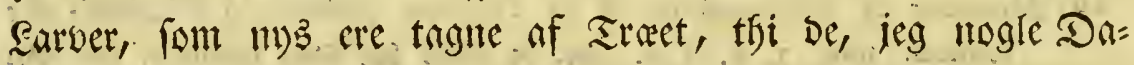
ge bebarebe i ell sejec, vilde eller funde iffe ubfproute no= gen Saft, i hoor mangfoldigen jeg rorte: og tumlede ben; og atter, $\subseteq$. $32 \%$. De, fom man nogen $\mathfrak{T}$ id bar bebaret $i$ cn 2effe, tabe Formuen at fpronte Denne Broffe, hois Rilde: Bald npantwivlcligen udterres, naar be iffe leve $i$ Dent frie

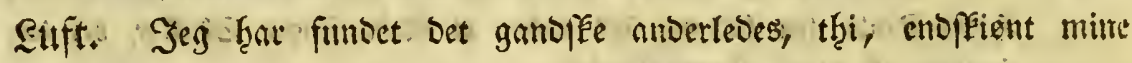

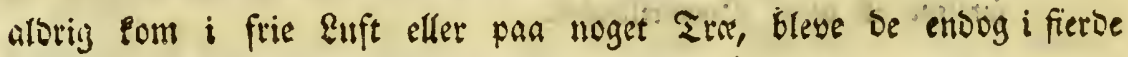




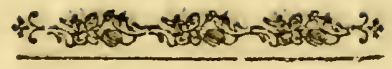

\section{5}

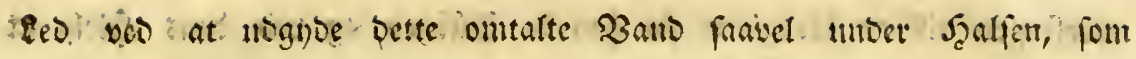
fla be antre anginne Etroet ofte $i$ Duerflesighed; bet. beroer nit,

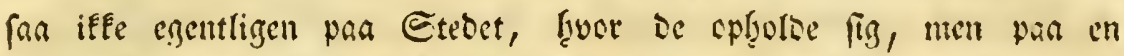

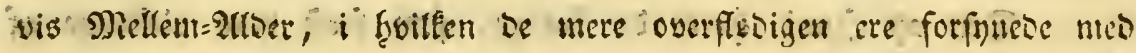

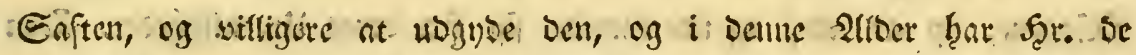

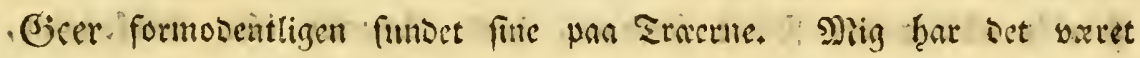
Iet at formage mine Earver til at fprevte fra mesen moer Scalfen faa tit ieg virbe, chlog de Turbworne Port fer beres Jnofwinding.

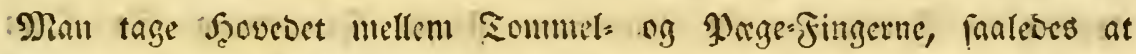
Munbingen og Etebet, byor 3andet ubipringer, venbe i Neret; Earven firrber Da of all פlagt at glibe 110 mellem Fingrene; man ma ifese give efter, og $i$ ell til to Mimiter liblater bon fureft en

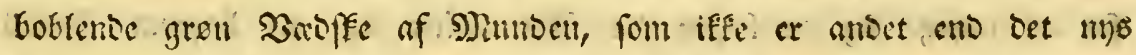
fortarede Dile=Smul; fom ben opbreffer; Dernaft, Iloprenter Den af Etrimen mot Scalfen fire Estader î en sangoe af toly til fior= ten ₹onnmer fire til fent (Bange for at befrie fig fra ien cuet= finngende suang eller Fare. Det forfanes fig; at de fiofte 1to= fpreutninger ere noget fuagere end be farfte." Det ex ganet mig

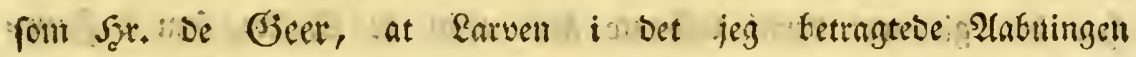

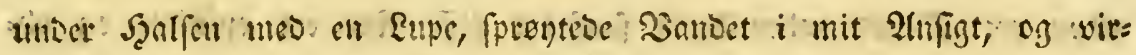

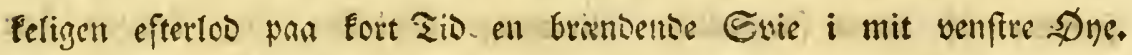

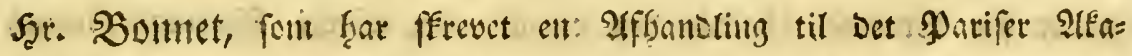
Demie, for at bevife, at senme Caft or et yirfeligt açidum, figer at Den er fearp, fun og Vibende, og at Dan mutigen tiener Gom: 


\section{6}

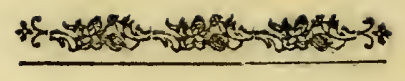

merfinglen til at Elobgitere fin fraarie Pribgen; og ved bents Shielp at aabue fig en llogang af famme.

Den 27 Suni forte be Earver, fom forft yare thlomme af

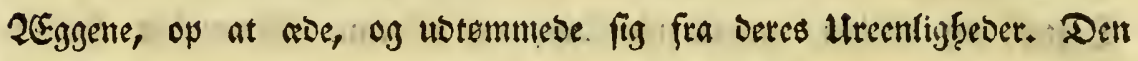
fmufle nblesgrome Farve forjuant, heele. Eegemet bles Merpesbun, og Earven felv mere urolig and frobuanlig. SFle at woe meere, Farvernes Forandring paa en og famme. Şut, og en uroelig : Sm= fryben ere Forvarfeler for Earvernes fretfe. Foranbring fra : Sim til Sonmer=frigl. Det ferfte f̧ac fut (jouno i Det, at Rarven og

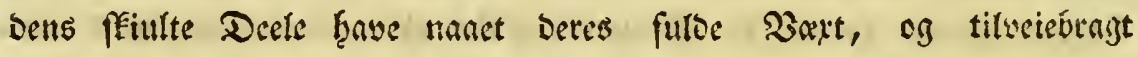

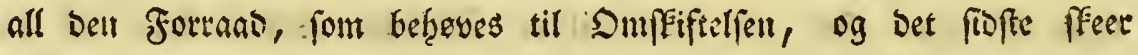
for at ubfoge fig be befrevente Materialier og ent bequem Mlabs

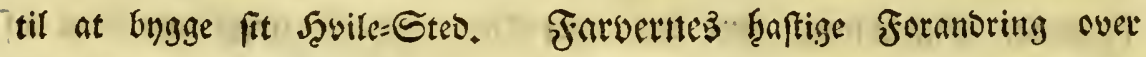
Earberues freele Eegeme foreftiller jey mig faalebes: Den Fabe, fom Earven fribinttil f̧ar mbt, haar Deels tient til at ubvibe og Howifle De ablfillige Dele, fom Ulogiare garvens og Den tiffommente Som= merfugls : Serabning, Der allerede ligger inben for $\mathfrak{E a r b e}=$ Jुuben; Deels bar Den gienment bertil beftemte: Sine affat be Safter, fom i Frem= tiben ere Dytet neswendige; faafnart biffe Fat ere opfylote og $110=$

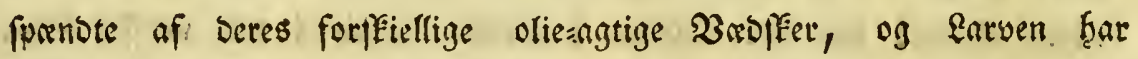

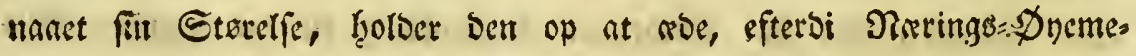

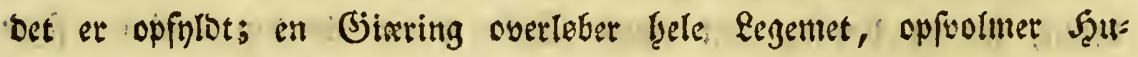
ten og foranderer bens. Farve; Den gronne Şut falmer, briber fmut= 
fimutig, gutul og: rod, og endeligen blod=rod. Earven foler noget, fom oriver ben til at Uotomme fin fra $\mathrm{en}$ Deel of biffe $\mathfrak{B a b f e r}$ og det paa cal Maade, fom en ligefa, forunderlig, fom ben er overeenftemmende med Dyrets Seftemmele.

Den 27 May war ben Dag, paa fiviffen be ubfont af 20 get; be baat altjaa ganoje nove anvento en Maaned paa at fomme i Etand til at aflegge beres Rarves SFabuing, og efter en Duale af

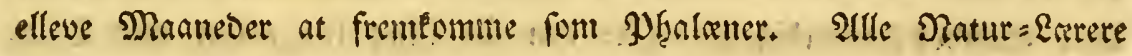
Grave vel tifferevet be flefte Sulfefter, eller de fer forfte Filaffer en trebobbelt Stano, nemlig Sarbe=, Suppe= og Sufeft= Sugl= Standen, men

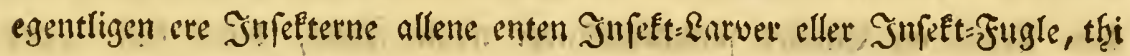
Den antagne Nellemfand af Puppe er ifepe en Stand for fig, men

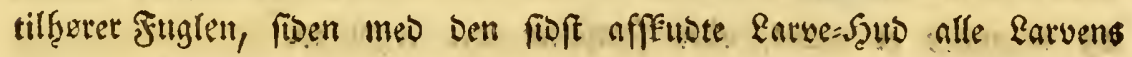
Decle ere medfurde, og Soumerfuglen meo de bent tilfommende Srganer fees it Puppen, fom egentligen intet andet et eno ben uns

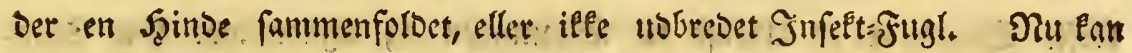

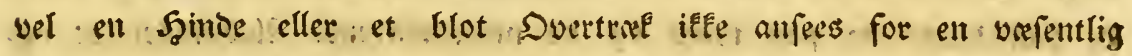
Forandring $i$ en Seabnings Silftano, eller Hogire et nut Ereatur.

For befto bebre at, funne tilfee Rarvens foretagense ved fiur Forvanding fra Earve til Phalcene, fatte jeg ben $i$ et Biras, og

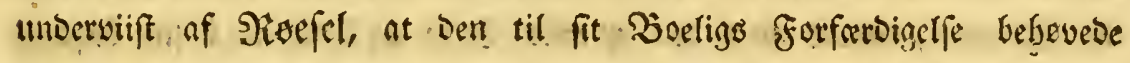

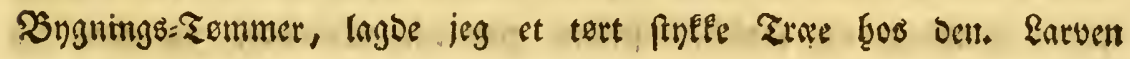


siifte fint fin Fornenerfe suer fanme web at befole set owen os

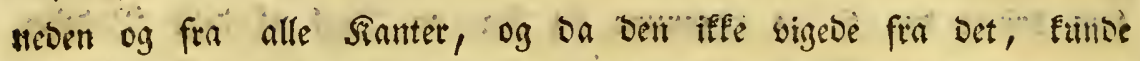

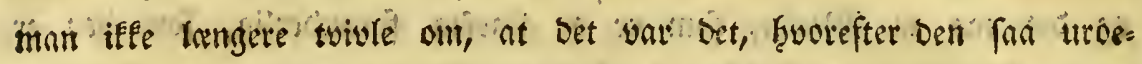

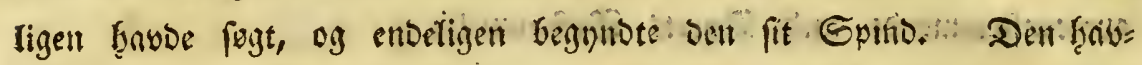
De hemffut en Deel af fit Eegene unber Streftumpen, sg Man faae

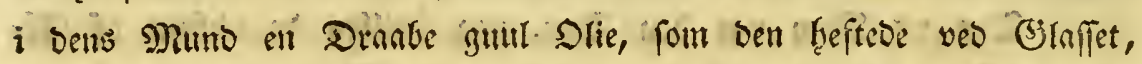
og Derfra brog en fiin Slic=? Trano til Den mobjatte. Sibe, fivor at=

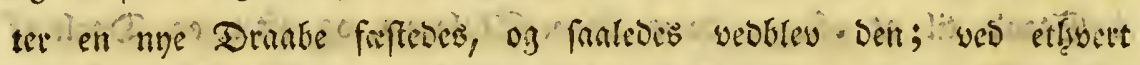

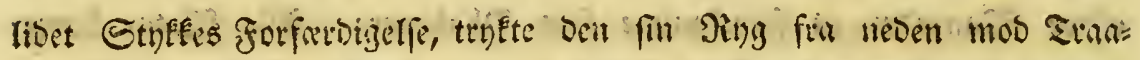

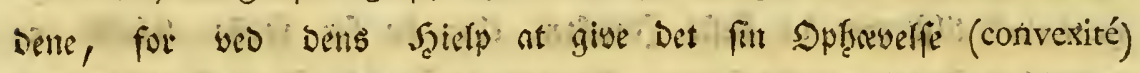

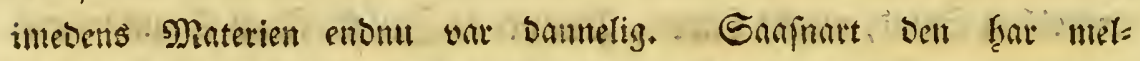

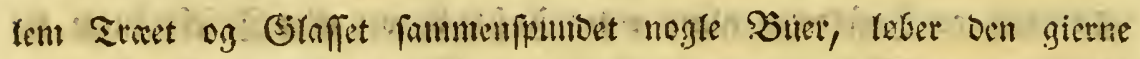
frêin og tilbrige giemem biffe SHer, og enten forlaber Dette Epino og begyitiet et andet, eller foutfetter Det begnndete?

Dette nye Boelig bliver oprtift il en regengtig figut: Det

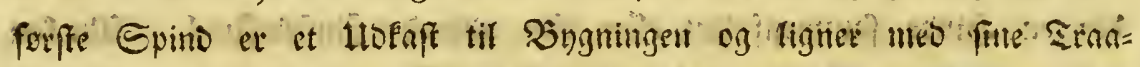

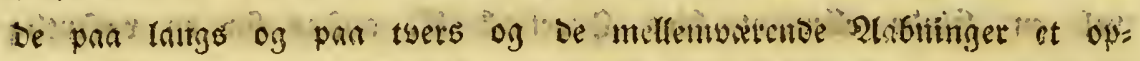

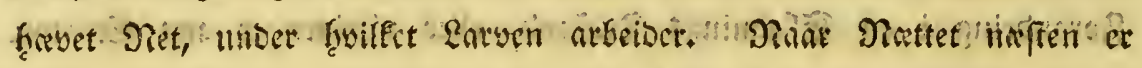

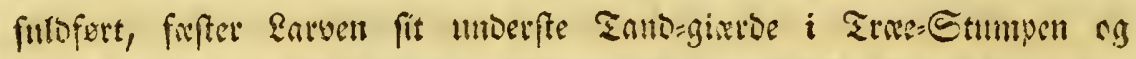

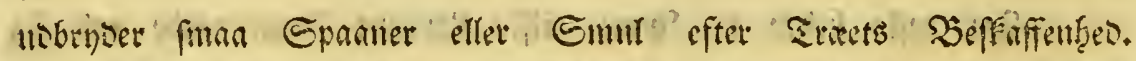

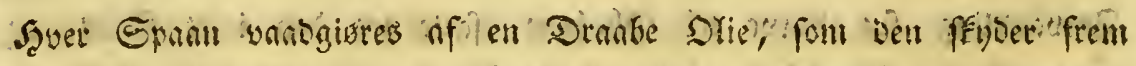

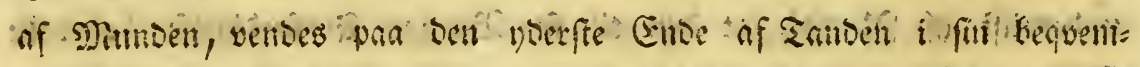
mefte Etilling, og fraleses preses gicinem, Epindet, ong then fro 
faftétines; beepa begynoer ber igien at fare nogle Tranbe, at brnbe ent Evan og at fline; og fantedes i nogle Dage vedblia

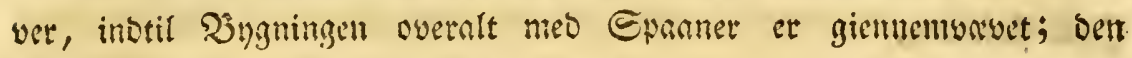

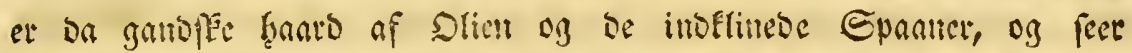

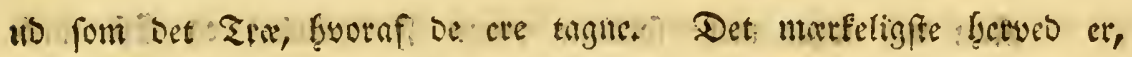

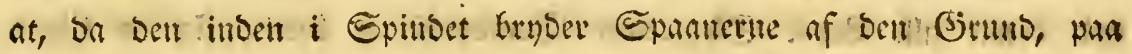

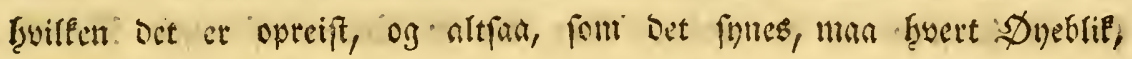
for inben fira at Horeffe Spnanen og then til at overfline Epint=

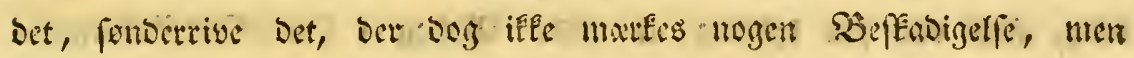

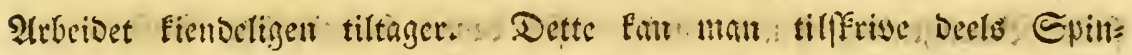
Sets. Efafticitiet, Fyorved Det gives efter, og igien famter fig, beels

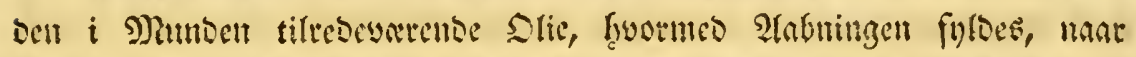
Earven braget fig tilbage. Enom Inange Dage, efter at foufet tib=

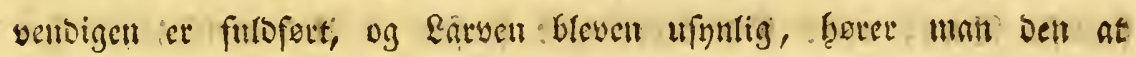

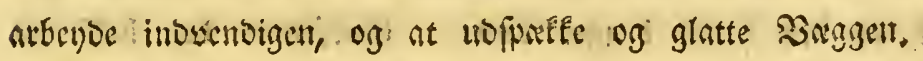

Shwortil affe biffe Forbetedelfer til benne Doergang fra Dum

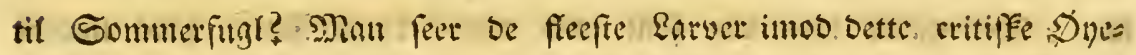

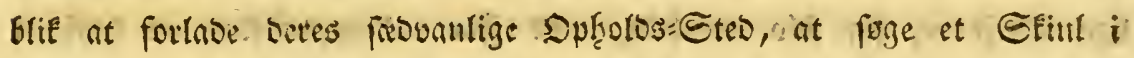

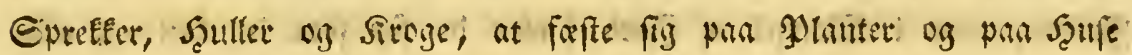

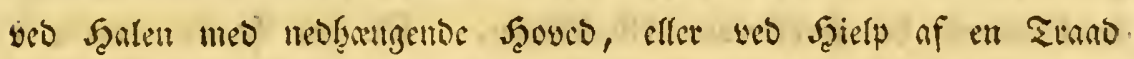
mist over. Deres Eegeme, at ernbe i Jorbert Hoen at gire noget. Epino, cller at inopinto fin usen for fanme, at blande $i$ Epina

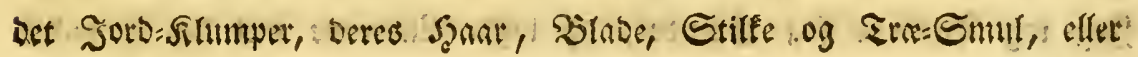

$$
\sqrt{1} 2
$$




\section{0}

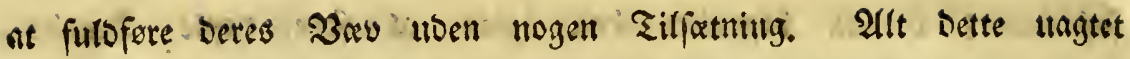
fatte bog nogle sibet til, andre Derimos giennemgane bet IIDen all forniftaltning.

San bel noget mere fortiente vores forulloring og (5ftertanke eno benme alle Rarve=Slegternes Biemienelfe at berede fig en Grab for at henfobe til et erolete Riv; bet er Enoen af nogle. Maanes

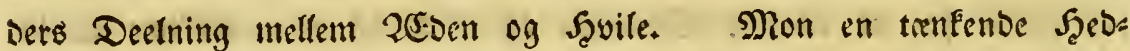
ning, fom var faa Inflelig at finbe $\mathfrak{B}$ eḩag $i$ fine Meoffabninģers ftille Betragtning, feulde Punbe tilfee benne befinderlige woforb tisen at Enfte en enten yoninggende eller fuigrende sante paa fig felo?

Mange Rarwer betiene fig $i$ beres Spinbing of be twenbe forrefte Been; vores Pile=Rarbe gior Ferfra en Unotagelfe; jeg fane nlorig mertet, at Den bertil bruger Dem. Enoffiont Den ḩelft vil bygge Der, f̧uor Den forefinber True til Şygningen, optelber Den fig Sog iffe lenge Derefter eller bliver famisuilo $i$ Mangel af fam= me. Det gaaer med Den font mes andre $\mathfrak{E a r v e = \{ r t e r , ~ b y i s ~ n a t u r = ~}$ lige Drift er, fig felv, overlabt, mod Forvandings=?ioen at frube i Jorben, men $i$ Mangel of Jorb fielpe de fig fom be fan og alli= gevel thofere be foreffreune Hoviflinger. Ft par f̧avbe allerebe $i$ Glaffet begyndt at inbfpinde fig imellem nogle pile:Sblabe, ferend jeg bley ibet vaer, og fravbe, forftraft Dem med Det beberige, Fine. Jeg. tog Slabene fra Spinbet, og lagoe, Rarverne meo bet begnnote 
Epind $i$ en Zeffe, men fom de engang f̧avoe, forento be forefantor

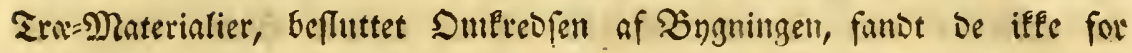
got at gire nogen $\mathfrak{A}$ abning for at bente Soummer, men fuloferte Deres boelig uden minofte Srex=ßrer, faa tret og fuldofommen, fom

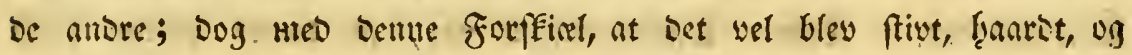
ugiememfigtigt, men ev fuld fa b̧antst, fom bet gientemtemrebe, og

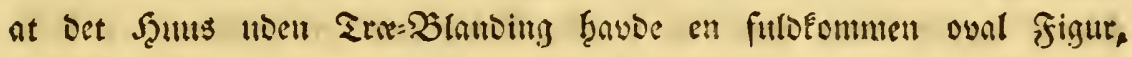

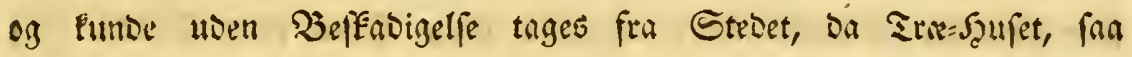
vidt four Det feitlte Det finfle Tree, fyorpan bet var bugt, ingen

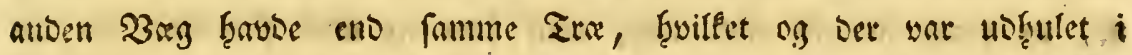
en Syal, paffende til Den tiffommende puppes Etarrelfe og Efab: ning, og bavoe forftraft alle be Epaan og Emul, fom til Bygs ningen yar meogaaet. (5il, foum imoo mit Bibenbe var fluppet tho af Bjlaffet, ḩaude mibt paa et Ege=bord opfert fiu Sioffe, og efters 10o, Da jeg meo - Mingt og Mene weo Şielp af en Siniv bortbred Den, en fandan oyal Şulfted $i$ bordet. Şeg fonderrey ell andens Scuns, fon, da Den ifle bavbe forefundet Tre eller Silade, yar fammenpundet of sen blotte feie Slie, og benlagoe Earven $i$ en 2effé; Den bley nogle Timer uroelig, enbeligen begntwote ben at fygge. et nyt Seturs, og bertil at betiene fig af bet netwerende zemmer.

Det vilbe bave weret intertffant at tilfee Snfeftets Foranbring Dag fra Dag inten for Soffen, f̧wis jeg flavbe funbet overtale mig til at opofre fan mange Denne. Nysgierrighes, men ben roelige For:

$$
\sqrt{3} 3 \text { nevelfe, }
$$




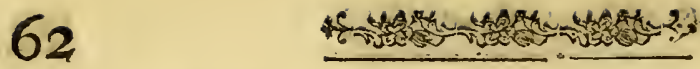

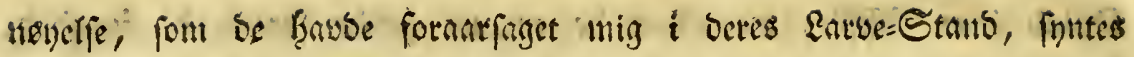

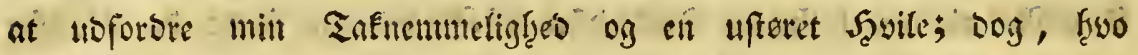

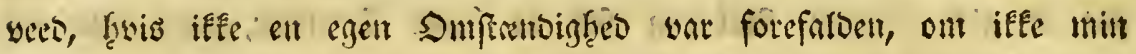

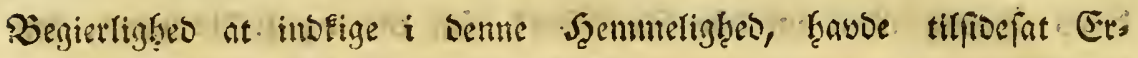

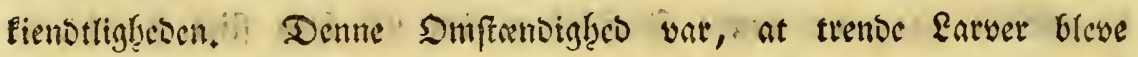
rooe, og Erete oinfring futb af Utwe, fom te eurige, begnnote og at fpinbe noget, men ware ey i Stant, jeg veet ely froorfor, at fulto=

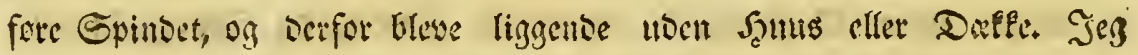
twenfte, at se ware upaffelige, formobentligen of Biagueple angreba ne, og fuat maatte omlomme, men blew sy lioct fornenct,. Da itts tet war oum bet ferfte; og bet frofte en fecller feeede, og jeg par biffe aabenbar fane alle be forntbringer, fom cllers foregane Gemmes ligen og $i$ Matke:

De rete Munben og Sgoucbet rigefom be antore fpindente Rarver, vertede fin langiom fia ben che Sibe til ben anoen, og bef̧obte i hrogle Dage Den blobreos Farve. Den 20 Junii bes. gunte beres Paltbe og bet everfte of be tvende forrefte Jinge at. llive grwane; ben ftore Aare, fon leber langs meo Divggen, faacs

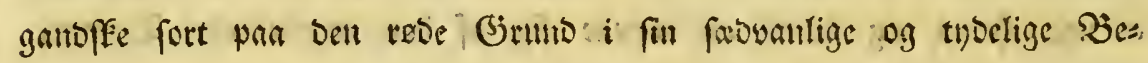
vargelfe; Den flod:rebe Farve forvandlede fig i bet blege; bet gran=

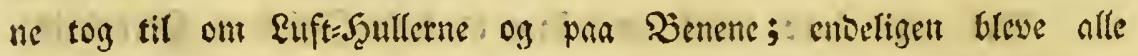

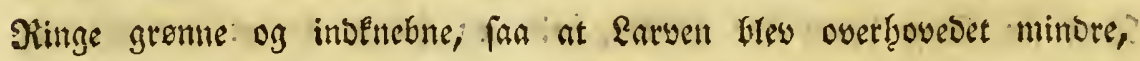
men. i Mibten tyfeere, og bebregede begge, Enber langfom og Mben: 


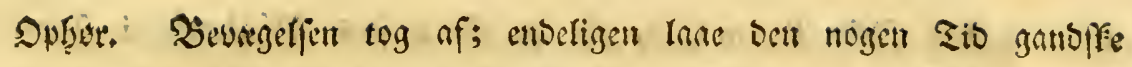
fitlle, waltede og rerte fig atter, og igien frifle.' Dens Trimbfieb tabe tes, og pai Cibcrue blev ben Hao; noget beiefter yenote ben fig oni. para Piyggen og bley averalt far inbferumpen ag: roelig, at jeg

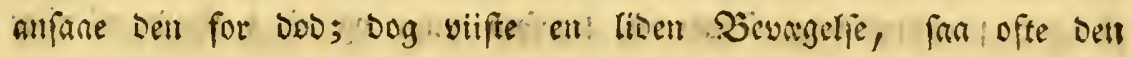

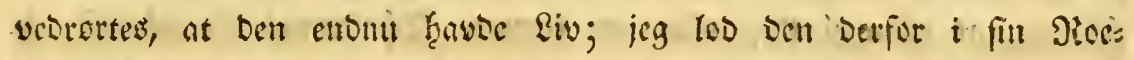

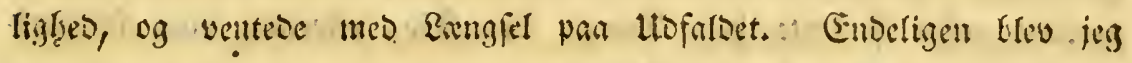
om Morgenen. Den 22 Sumii iffe: liset forthoret, Da jeg, veo mith

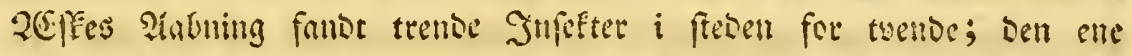

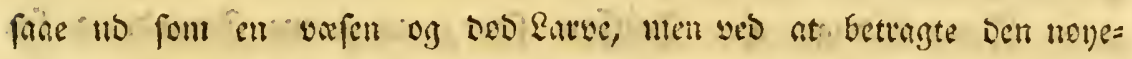

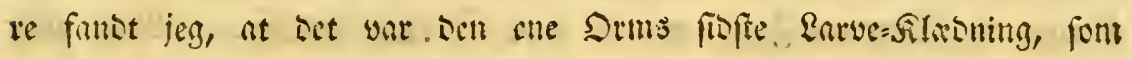

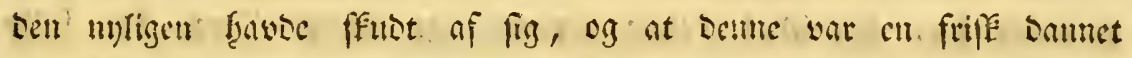

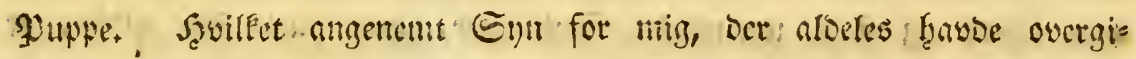

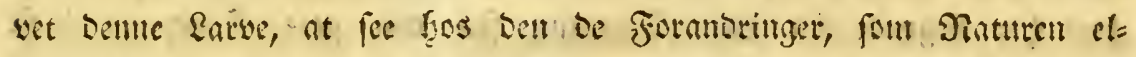

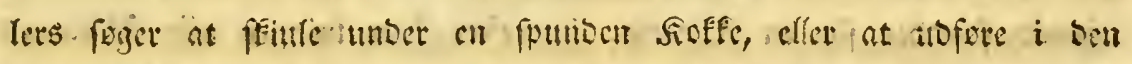

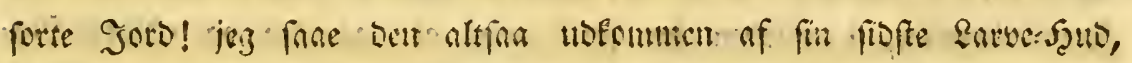

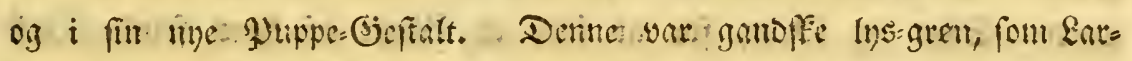

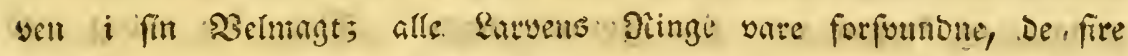

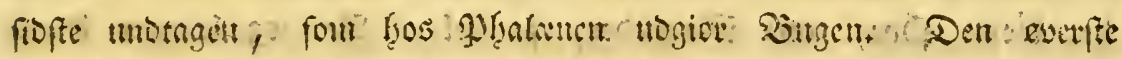

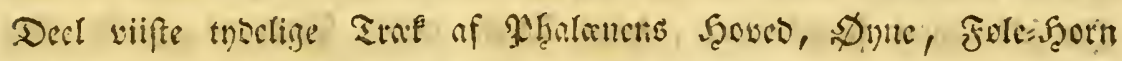

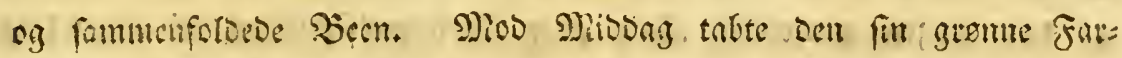
we, og kley, violet, iog mod affenen yne Den weetfe Ded fulfout

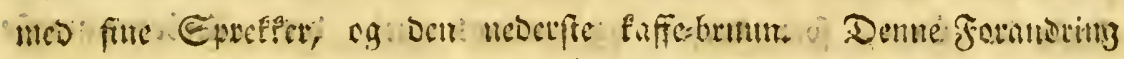

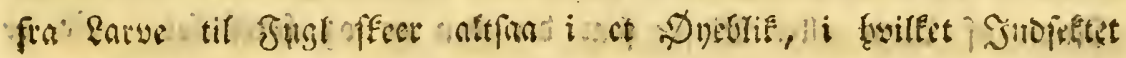
affiv $=$ 


\section{4}

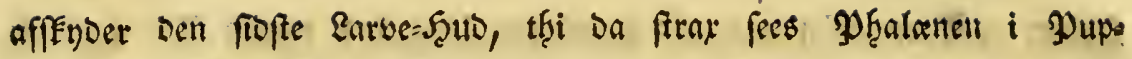
pen, og befgever intet vibere til at flyoe, og at aule, ent at be garte og blabe Deele fintfes og fartbes veo be overflabige Safters Ulobumftititg $i$ en efter \&uftens Barme og Siuloe, Fugtigheo eller Sorffed proportioneret Tibsum, ligefom man og er $i$ ftano til efter

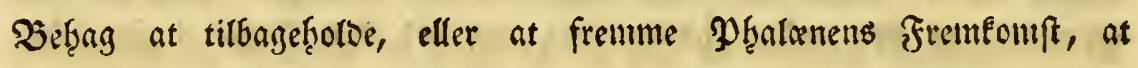
forlenge eller forforte bele Dorets Eeve= Sio, efterfoll man forftrefs Eer ben mere eller minore Barme.

Deal finbrige Şr. Şuffon figer, at Deme Foraltoring fra Earve til Sommer:Fugl er en Mye Frembritgelfe. Man viloe forundere fig, fyoorlebes Denne berommelige Natur= Exrer Fan falbe para benne og flere ugrundede Sxtringer, hivis man iffe viofte, it f̧au f̧ar begundt fiu \&ere= Bugning fra ben gale Embe, og $i$ fit Seoved fimedoet fig en Theurie, b̧uorefter ban twinger alle నaturens Frembringelfer, $i$ Steben for forft nene at paraagte og nolofle Na:

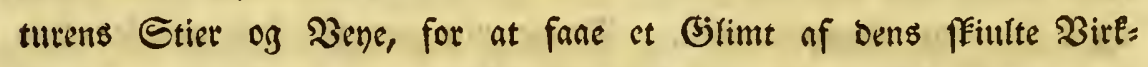
ninger, og Derpaa vove fine formooninger. Man feer ettoog med Det blotte Soye, otte Maaneber, ja vel et 2 tar og lerngere foreno Subfeft:Duret fremeomuter, Dens Deele unber Puppens Scinde Hoen

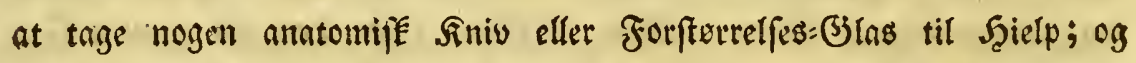
ved Shielp af diffe feer man Dem endog i Earven fely, eller $i$ bent frybenoe: Drm, fom Derfor og nesunes mes Det allerbequenumefte Navir af Earve eller Maffe four Det ben er og foreftiller. Er be 


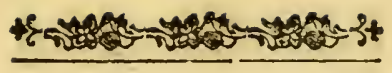

65

Eommerfuglen en nye. Frembringelfe, eller bens lange tilforn tiltits. beurrende Deeles Hovilling? Earven Ran etrodg iffe figes at for: vandles til Puppe eller til. Sulfett=Sugl uben i Den allervibtleftigfte

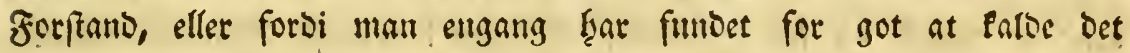
en Forvanoling; thi Den forvanbles faa libet til Puppe og til Sommerfugl, fom en nubfprangen Wlante til Slomfter. Eommer: fuglen med alle fine senmer ligger ligefanfulot $i$ Rarven, fom Blomfteret med fine Deele i $\mathfrak{P l a n t e n ;}$ leg Beggetets bilave tilfite,

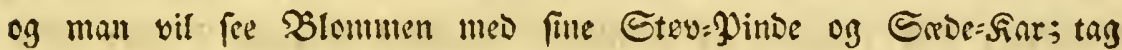

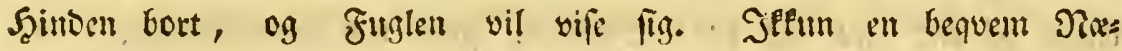
ring og 2enrligt therrwes til at thovifle begges ind frylleoe Efign=

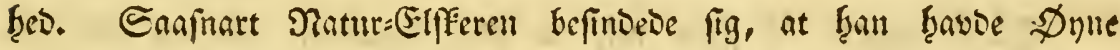
for at paasagte, og Fingte til at frapille, fanbt man Summers Fultelens Binger, Tunge, og Feletgorn fonftigen fammenfolbebe un= Der Rarvens twenbe fartepte Siinge, og f̧ines fer lange 3 cen fam=

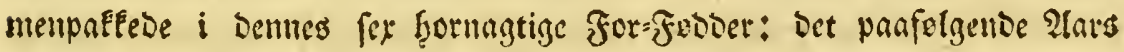

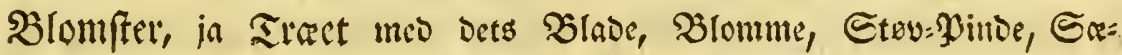

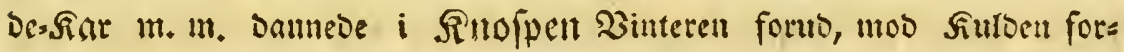

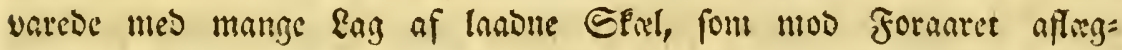

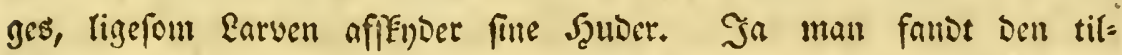
fommente Papiftions 2Sig enbog $i$ bens Rarve; altaa vare be og allerebe $i$ bet 2eig, byoraf Earoen fremtom, fratedes form jeg i

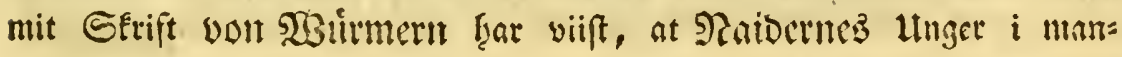
ge Eod ligge fEiulte $i$ Moieren, og i ocres entelte Deele efterf̧aano 


\section{6}

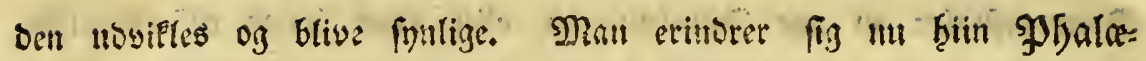

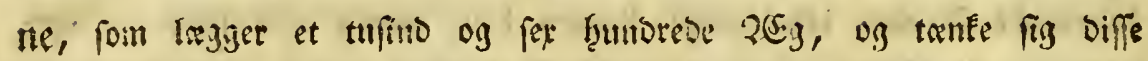

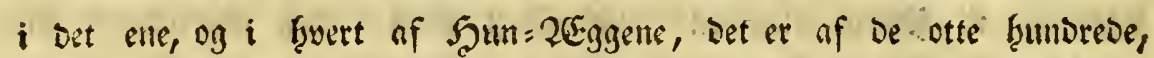

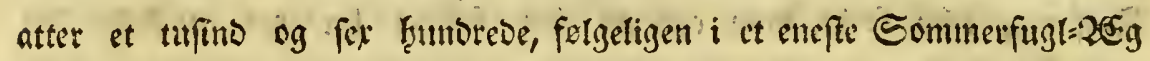

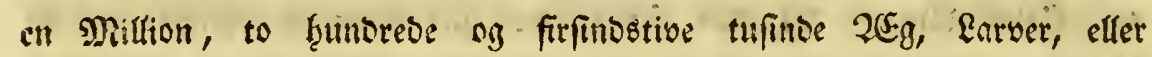
Sommerfingli, fyad man vil, eller bette alt tiljammen mes alle be: res ind og uovortes Deele. Dette er forft i anden Sisting, og firmfler allerese vores Snobitoings = Sitaft, ffiembt Fonnft og

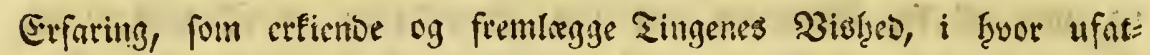
telige be ento are for yore Sanofer, giore bet uimoofigeligt.

Şr. S3onnet ${ }^{2}$ ) figer vel, at alle Sinfefter, fom ere unberfas ftese Smffiftelfer, $i$ Den fterfte Deel af Deres Riv, iffe ere af no= get Sim; men, Da 2erggene allerede findes i Earven, og folgeligen tillige se andre Deele, fom beftemme Sivnnet, ia man enoog af Det thovortes Enw, of Earvernes forlfiellige Etarelfe, Ean vide, frsilfen er ban og boiffen Gun, faa fan oe, ffionot Parringen forte ganer for fig under frue og Eommerfugl = Seftalten for ben Tio fan libet pan= ftanes, at fattes Sirmut, fom et Fyert nubet Dye ba ferft at blive

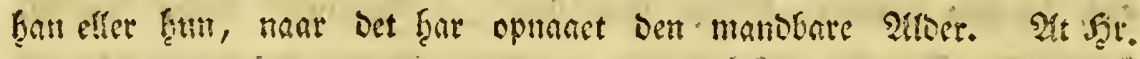
De Sbiffon antuger, at be $i$ beres forfte beftalt, og fon Earber,

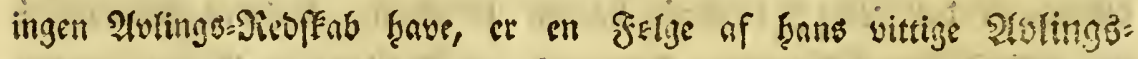

22) Confiderat. fur les corps organ. vol. 2. p. I35. 


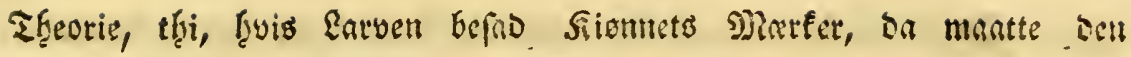

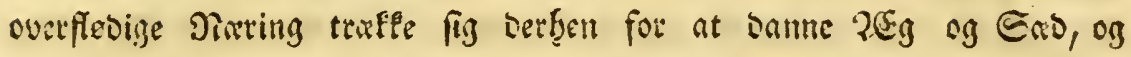

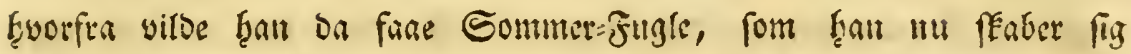
af Earvens overffigoente Nierings:Saft? Dueralt bringer Foranbrin=

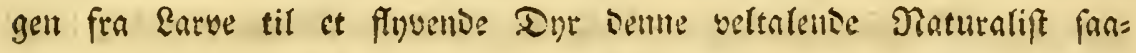

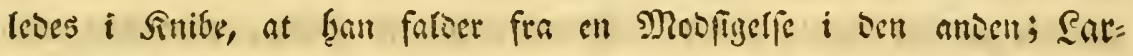
ven, figer fon: bituer til Y̧apillion, fordi bent, Da ben iffe har noget Organ, fom Ent modage Den doctflobige Naring, iEEe fan frembringe fman organife Detfor antager Den altio virffomme organifEe Naring ell an= dent Stikelfe; $i$ bet bent fammenfetter. fig, og efter be for= bindelfer, fom finde af Lurvens Geftalt, banner en Sapiffion,

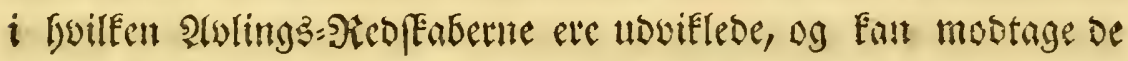
Diganiffe Decle of : Narings: Saften, fom banne 2eggent og Artenis: entelte Sfabninger, cller med at Srb, fom virke 20 fingen. Sommer $=$ Fuglen er intet andet ent in handelig. Feembringelfe of Dent overfedige Sarring, og intet thoen et Midoel, fom Raturen anocnder for at Eomme til Dent reetle Frembringelfe of Earber; og de entelte Sfabuinger, fom av: lez of Sommer Fuglen, bor iffe bre Sommer = Tiligle, ment Earocr, fordi dat at Sarvell, fout bar taget mod fober, ag Denne PRrringens organiffe झुartifler gave afformet fig efter Raroenz bieftalt og iffe efter Sommer=Fuglens.

$$
\Im 2
$$




\section{8}

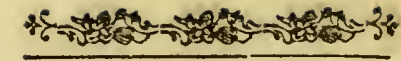

Snart féulde man falbe paa be Fonler, at benne beromme: lige Mant aloriy bavbe tilfect Earvernes Iffelroning til $\mathfrak{P}$ apillions

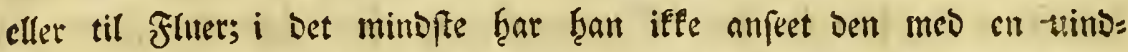

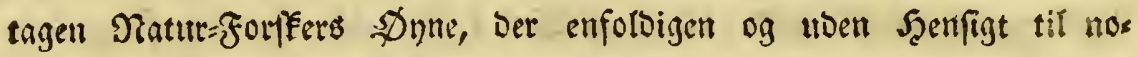

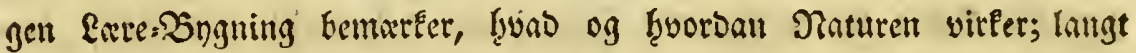
minore fertud taget anders Semaréelfer til Şielp, eller felto med

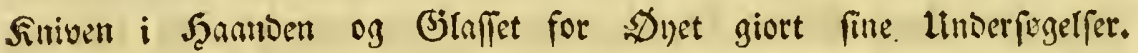
Saan viloe ba med Siwanmerbamm, Eyonet og ande allertose

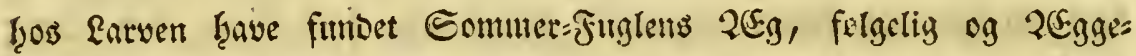

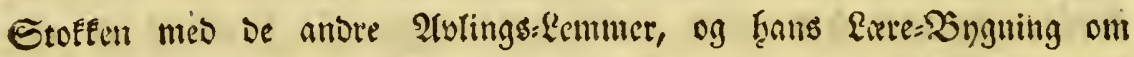
Afforminget, $i$ hyor forvitret Den er, vilde Derved iffe hrave blevet ubegribeligere. Dat Da han antager, at Sulfefterne $i$ Deres ferfte

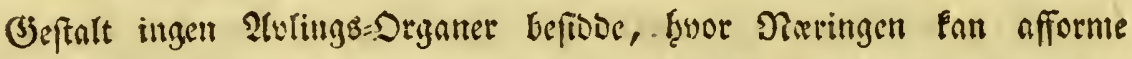
fig, og berfor paaftaaer, at Earvens overfledige Nirerings= Saft affor: met og foreener fing til en Soumuer=Fingl, faa falber fian $i$ en non fens, tḩi fryorledes lan Den afforme fig til Deele, f̧vornf ingen Sormer ere forf̧antben, og, Da efter Şr. Büffontz Saģ $\mathrm{i}$ Earven ilfe are andre end Earvens Eentnter, i f̧uille Den organifeerte Dax: ring Ean formes, faa ber jo Saften, naar vi og fette, at frans $\mathfrak{L}_{\mathfrak{a}}=$

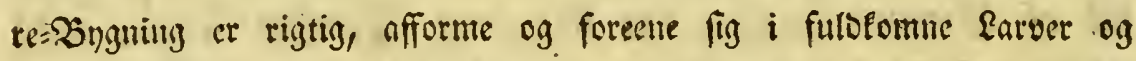
iffe $i$ in Seabuing, fom iffun tilbeels ligne Dent, Da be Deele, fom

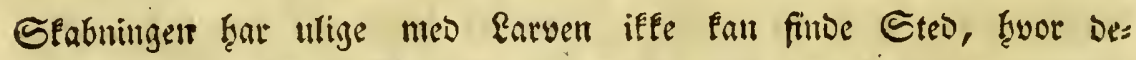
res Forner fattes, og fanbanne ere Dog Fuglens Binger, Fole= Scorn,

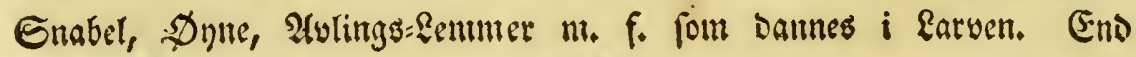


vibere feal be organiffe Yartiflet, fom frave afformet fig efter \&ar: vens Eemmer, foreme fig til Rarber; men, froot ffal Denne Eam= mempettelfe gaae for fig, Da 2tolings=Drganerne efter fatmme Natur:

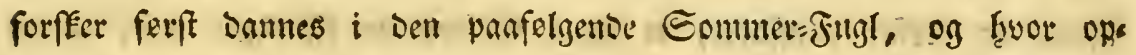

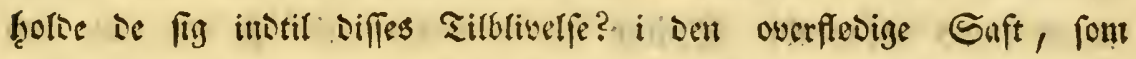
moo Diffe Partiflers Yaaftard at blive Earver, bame en Sommer= Fugl meo 2tulings=-Qummer; men, fuorleoes flippe be ind $i$ diffe? og fyorfor fan be iffe ligefan wer then at fantes $i$ bent, Dan ne Rarver, fom Wapillions; eller fyuorfor give be iffe; Da De i Nirringen have pafferet formen of car Sommer = Fugls Eemmer, ligefar vel unge Souturer = Fugle, fom Earver, eller, on be - forft tage Snotrne of Earve=-smumer, og Dercfter of Somumet-Fuglens, forft

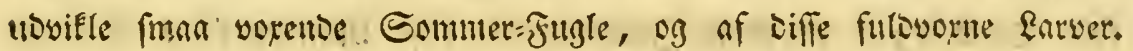

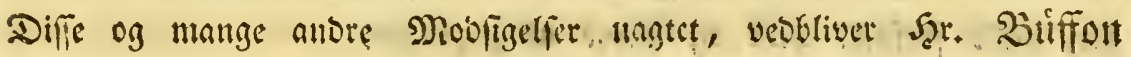
Dog fin Mening; Den Eere= Buygning Fian engang frar aulet $i$ fin Şierne, maa fremferes, om og be alminocligfte og uforanterligfte

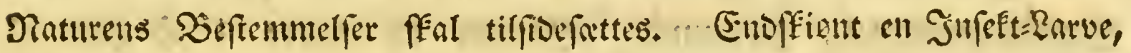
fan lenge 3 erven ftaaer, albriz̧ har, eller fan forvanble fig til alls

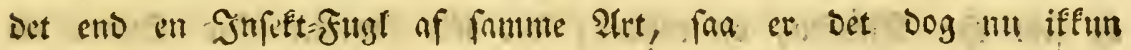
en Scenselfe, at Den bliver ell Eomnet= Fugl, hyulfet Dell, f̧at ben swerflebige Norring at taffe, folgelig, naar Den favose minore Dix= ring, flev ben vel en filue, en Ming, eller et andet libet Silie;

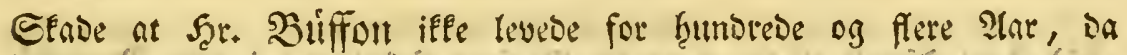

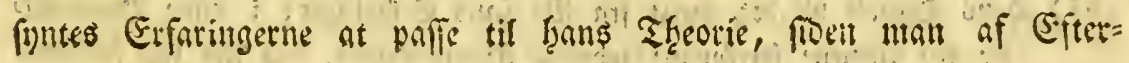

$$
\text { I } 3
$$




\section{0}

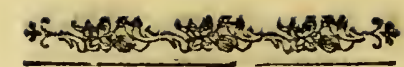

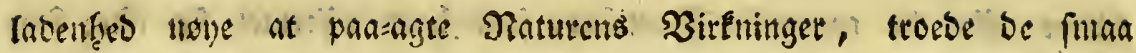
Durs og Shanters vilfnarlige Frcmbringelfe. Denne feger fian of alle Sirmfter, enoffient ḩele Naturen ftribet betimos, at optange igien, og berfor paaftaaer, at f̧ans Erfaringer elartigen vife, at bet ingen fortlo tilbatento Spiret (germes) ele til ${ }^{23}$ ); nt ber veb en tilfarbig Samling of organiffe Martifler maaffec frembringes li= gefa mange levente og voreribe $3 x$ fener, fom af en beftandig Ef

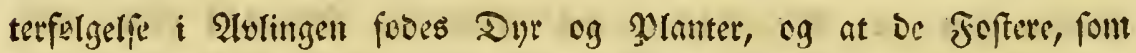

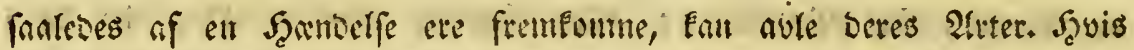
fra var, vilbe Naturens Srben blive Forvirting; Llvistgeo og Llfie

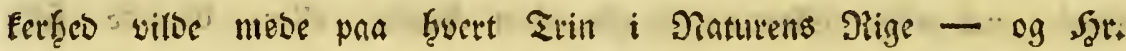
Bilffon, Der for at have noget befunterligt panbyoer 3etDen meo fuld Sillio og $i$ en fime Inoflxoming diffe Natur=fribige Sretnin= ger, villoe frafaloe" Dem og fertive ubefingeligen.

Jeg fiar $i$ bet Fereganattio allerebe befferevet, fruab jeg med Scfiwammerbant, Sieaumuit, Bonnet og anore i Senfigt til

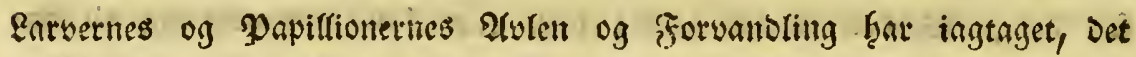

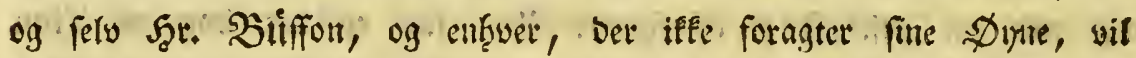

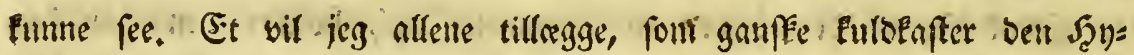

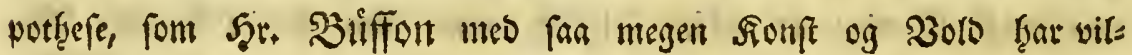

Det

23) Şants Theorie vil bet, langt fra iffe bans Enfaringer; I mine Demarkelo

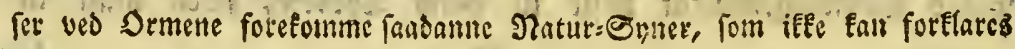
uben at antage fonlig blivende fig udviflende Spiret. 
bet aftuinge Naturen. Demlig Sat. von Satlet's Spongelfe. Şan fanot $i$ 2ङgge: 3 lommen uben nogen foregaaende, Parring be farfe

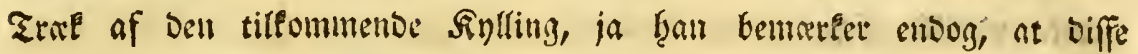

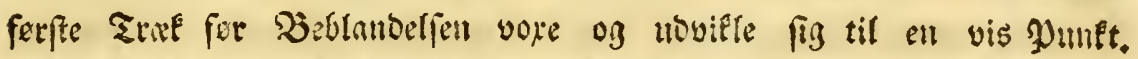

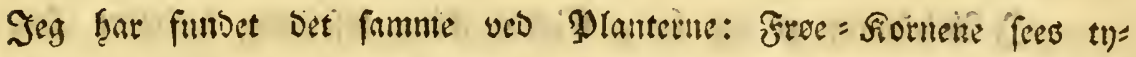
Detigen $i$ Sapslerne at vare Dannede, og at vore og tiltuge fe= rent nogen beftugtelfe peet er. Jig bar viift set be Sgerr. Bonnet, Slembley og ander, og entruer ton fetw fortentfe fig Det Enn, naar Gan-befrager. Altiaa ligger fofterets efler ben lille

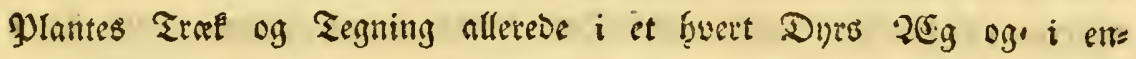

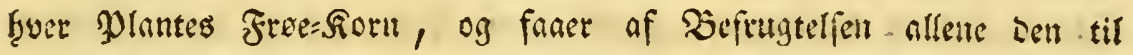

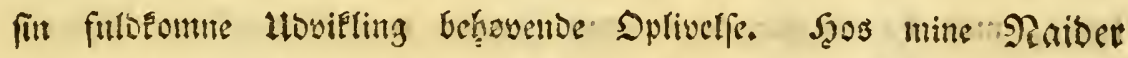

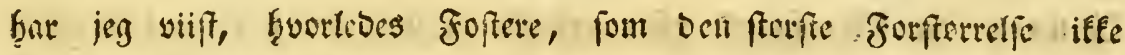
Ean fEimte, Hon Darring $i$ beres enecte Drele blive finlige og Dag fra Dag tovifles 24): ferft bliver matt fine Sver = Etringet

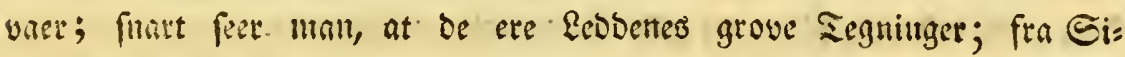

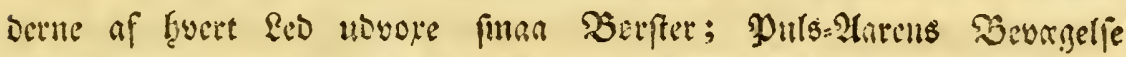

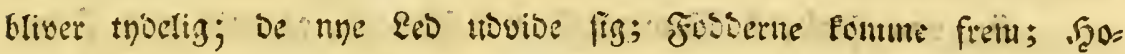
vebet bonnes; en. Enabel fremffubes, og bliver Dagligen lenthere, of endeligen fers Sonnene, og Hngethe ere fuldofonue og fartige at

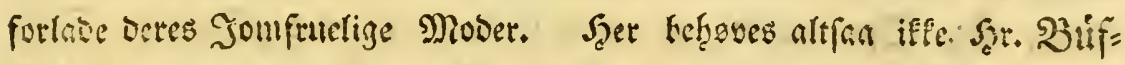

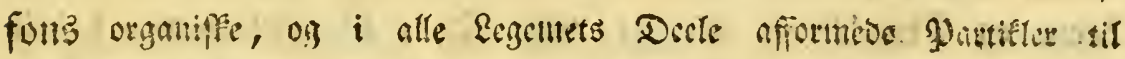

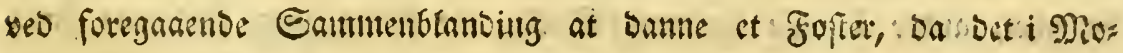

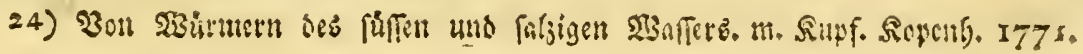


Deren lange tilforn er forhaamben, og allene venter paa en ubforbret Siodenhed, og en efler anben nebvendig Jactidelfe for at funue eno vibure thuifle fig.

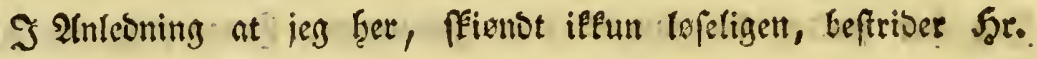

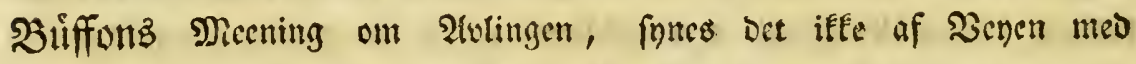
fan Sob at tilfiendegive mine Taufer cm Denne SRaturens viatigfte Formaal i Alminbeligheco. Den f̧ar intrigeret alle Tibers $\mathfrak{P h i l o =}$ fopher, oy botoer bell entum i Aanoe. Biffeligen vare be fomme

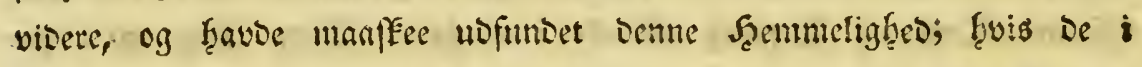
Eteden for at raifonnere, fravbe neve. F̧oldt fig til gode Sommer Felfer, men Da mantte de $i$ egen. Perfon utratteligen Ģave abjpurgt Naturen, og iffe, fereno efter crigolot Sunr, troet fin at forftaae Den. I Mangel af Dette ete Chimater opreifte, og De Songninger,

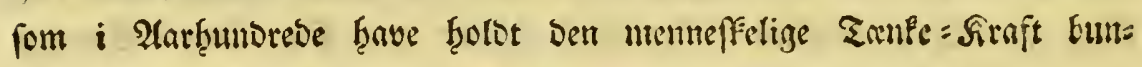
Den, ere wed en fifker og goo bemmerfelje nebftnrtede.

Şr. von Sallers Demrelelfe ved satgget, og min ves Froes: Sornet lagge uimobfigeligen for Dagen, at Fofteret cr til $i$ Mioseren

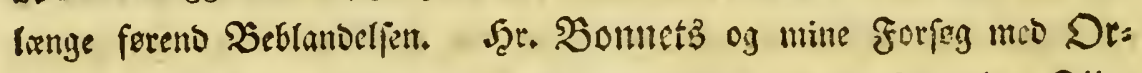
mene, Sct. Spalangani og mine Erfaringet med Snegle, Fitr= been, og Jraellnger vife, at nogle. Dnr, lii\} צुlanterne, $i$ fele Eegentet, andere i viffe Eegemets Decle, ere fylote meo faabanne fma Moleculer, fom man af beres formue at vore Ean Ealoe Spirer, 


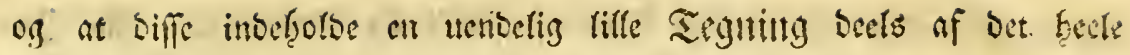

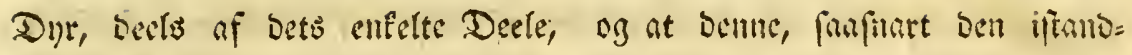

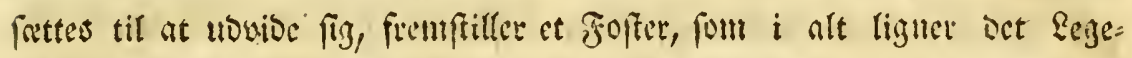
ute, i f̧uifect ben lane forborgen. Der bef̧eves altian intet uben en uovioenoe Sirnft til at jiste siffe foftere fonlige; men feviffen cr seme,

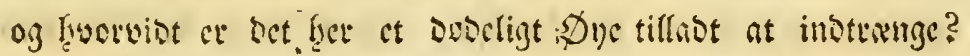

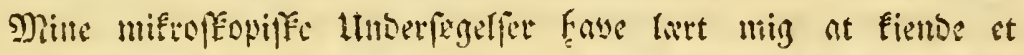

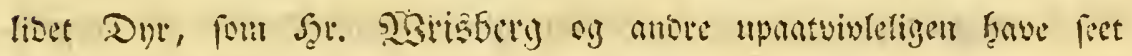

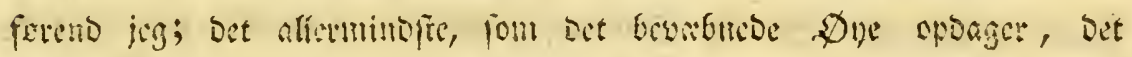

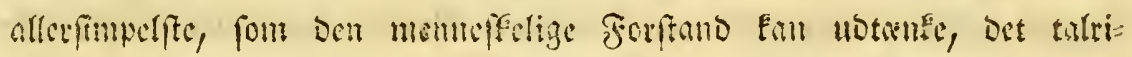

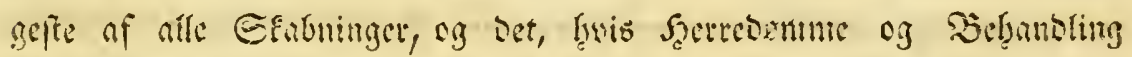

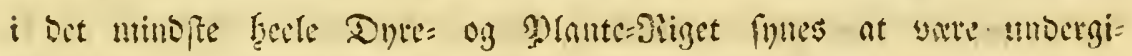
ven. Dit ei ell Elar Sounft, ber; nane sen ex cule, hal fin friz=

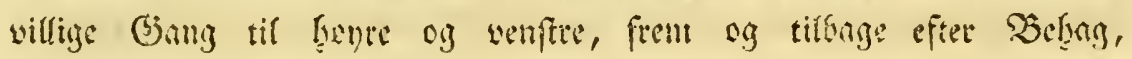

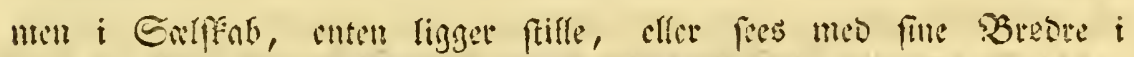

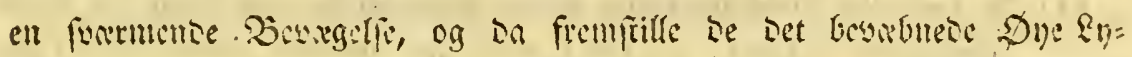

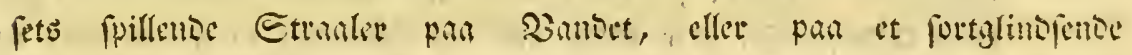

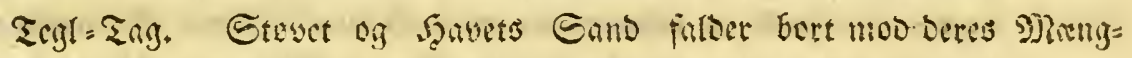

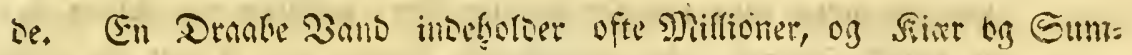

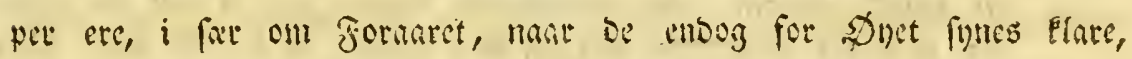
en furbfonmen Maffe of biffe Dor, Dit at it tevente Contumm

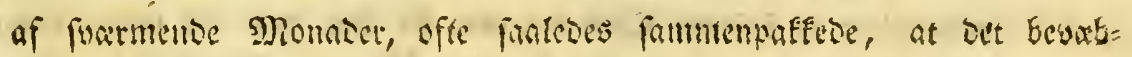

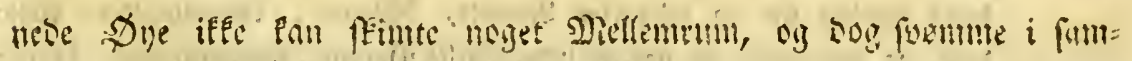




\section{4}

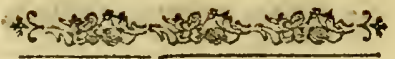

me anbre finlige og ufnnige Dur af forffiellig. Sterrelfe. Alle. Jin,

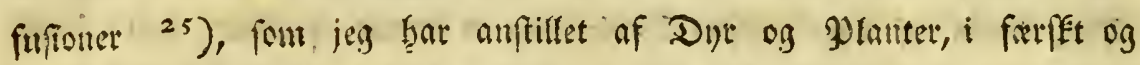
falt 3ano, have viift mig Dent i ferce og minore afutal. De

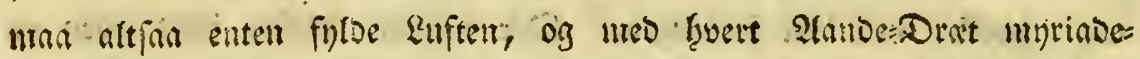

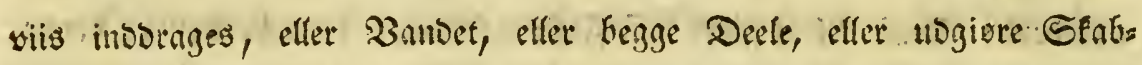
ningernes $\mathfrak{B} x$ fen, f̧uitiet er fanblinntigft, enbog fordi be ifle fees uben $i$ bet Band, byoor Moranter og onrifee Deele oplofes.

Diffe Monnder og anbre fige give $i$ mine Tanfer alle organi= fereive Efabninger siu og Bevregelfe, Hovide og ubvifle Dem efter de

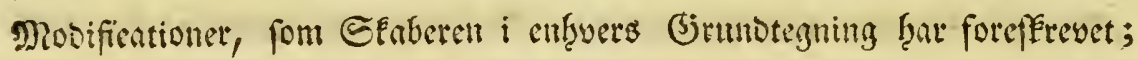
de brande fig med Banbets og Dixtingens Sorbiffe Deele, og i forbelo til en mintore ellev fterre Blanding thogire Eegemernes flyoente og fos live Miafler. De ete nforginengelige, fanyelfom Sfabuingernes Grumb=

tegning

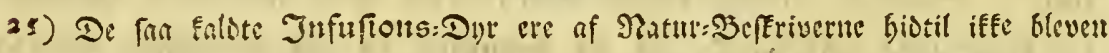
neyngtigen beftemte; jes bar $i$ nogle $\mathfrak{Z}$ far auwend mange Mretter pan

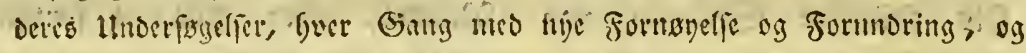

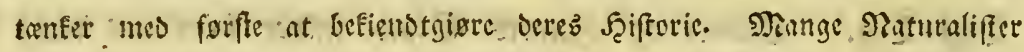

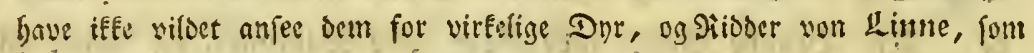

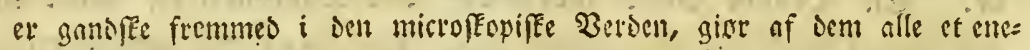

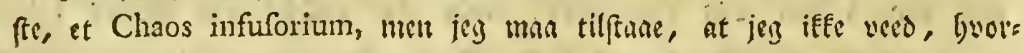

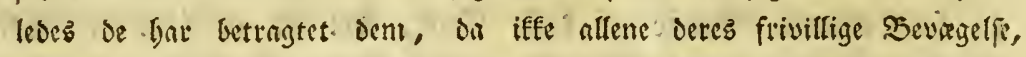

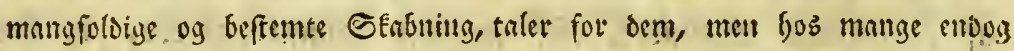

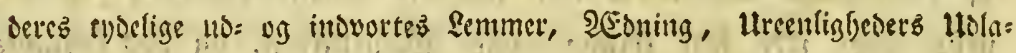
oelfe, og hos nogle en heften uimodfigelig porring, fuldefommeligen bevifer Dere Dyr:Sittighed. 
teguing; thi intet af alt, four falber i be menneferige Eanbjer, Ean eoclegge en enefte af bent. De tnbe Rivet, og blive levende igicn. De famle fig i enl livles Maffi, forblive fanledes ell ubeftemt Tio,

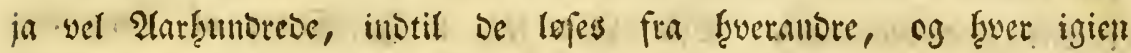
facer Riv og Friefged:

Det Gliver nobvenbigt at angive nogle af be Bemerfelfer, fyvorpar alt Dette gumber fig: jeg fage animaliffe. og. vegetabiliffe Deele ned Bicnnemblobuing at oplofes $i$ netformige Sainber, og bif fes rumbe cller ovale Minffer (arcolx), fom albeles ligne de om= melote funa Dyr, enEelt at Iestabes fir sen becle SNaffe, at ytx tee - en fientelig Sewrgelfi, og insen fan Minuter at nmpemme $i$ alle Bensinger $=6$ ), og farcoes fra ct subeg uorganife segeme at

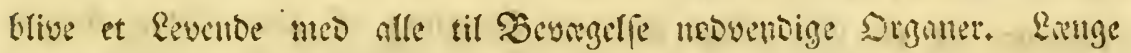
wilie jeg ifle troe mit Done, ment tunfte, at set jey anfane for Dafert, fom lesigionte fir fra Den netformige sjimbe, vare be fig

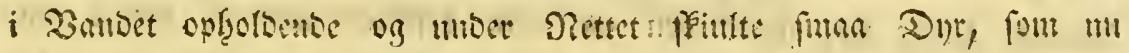

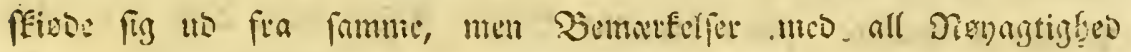
igientagne vilse iffe tillabe mig liengere at twisle par; at jo mit Sobe bavoe fect ret. Dijpe Dur fare enten enfelte omEring, eller famle ing $i$ beftente og ubeftemte Figuter, nomuc og ntoen Snoflixb= ning, eller inogane i viffe Eegener, novioe og novifle sell. S Dell $\therefore: 2$ ferfte

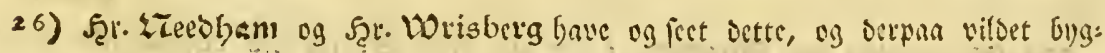
ge in fra unin gandefe forficillig, slycorie. 


\section{6}

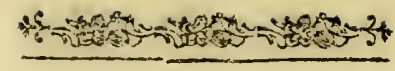

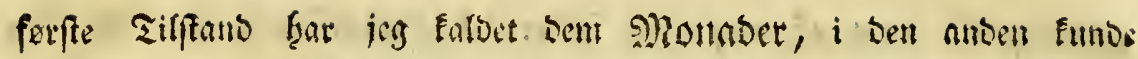

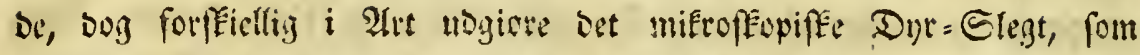

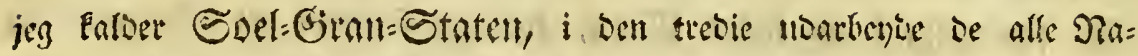
turens Frembringelfer. Dfte fare: jeg $i$ Sufufiener of Dys= eller

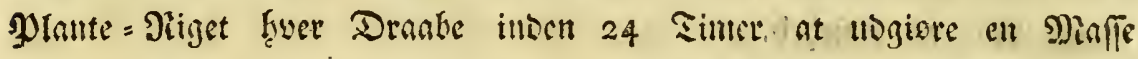
af Diffe funan Singler, woen at fumme merte minofe Bevrgelfe og Sugt; i Fort Sib panfulgte singlemes furrmente Sernegelfe eller Znanbets Bimting og utadelige Etumf.

Anbuingen af Merent pan Siot = Soumpene, bell poerfte

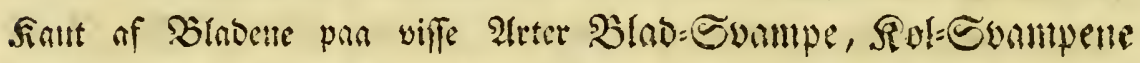
og allore ere belinge med ent fiin Stev i ulige Sabre; Denme er Dit yoerfe of Deres 3egetntion, ligefom Etev=Sinoppencs Mecl fios lttterne, ag er iffe andet ello en Samling of fman flare Sithler,

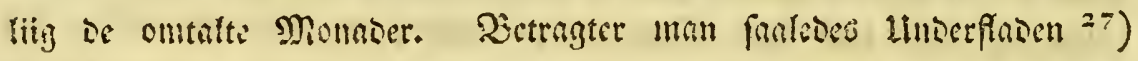

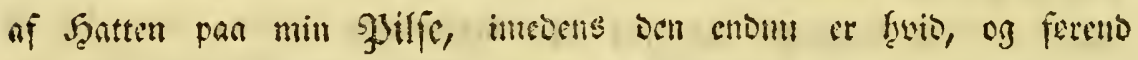
Sivenc anfue fig, fynes ien at vore befteet mes at imegat fint

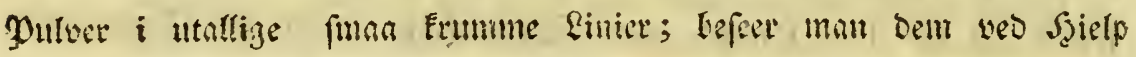
of Stapen fra oven, eller mibt for, fers ben fyele Flabe at vare be= faact med fiman Etienter, Dommese of utaltige fman Streger, nus

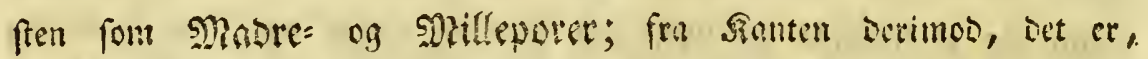
nane man forber Llnoerfaben fanleoes imoo Dagen, at ben meo Dyet giet en f̧origontal Einie, er ben ligefom runo af owermanos fimaa,

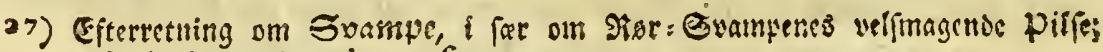
gis6enl. 3763. tab. 2. fig. 5 . 


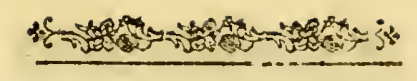

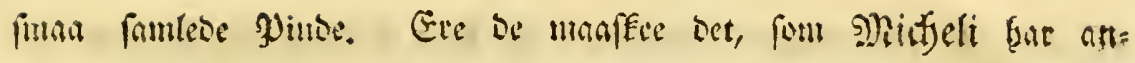
givet for Etoy=guinoe? Imet, byor libet paffe frans og bans Eifter

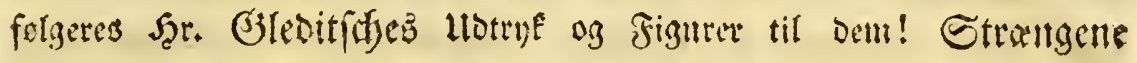
(filamenta) Eunoe of vire, men ingenlunde Sinopperne (anthera)

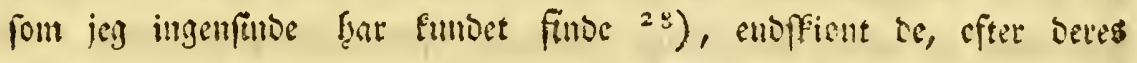
Tegninger at bemme, ere mange Bjange frerre og tyelete ent Etron=

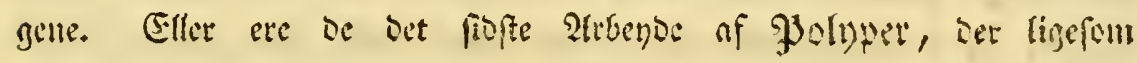

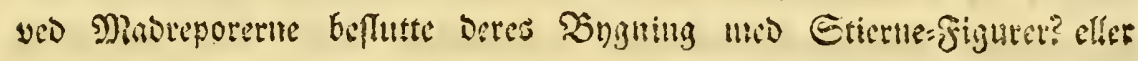

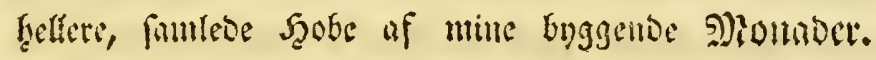

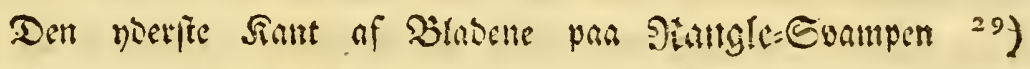

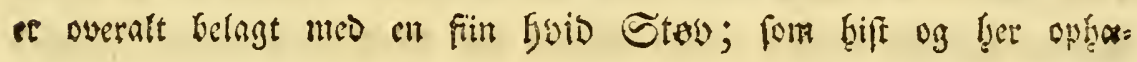
政 3 ver:

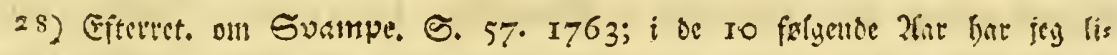

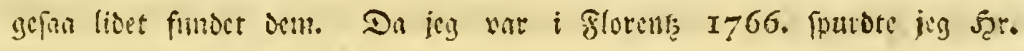

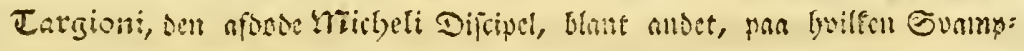

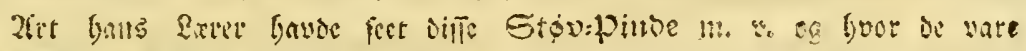

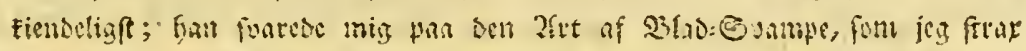

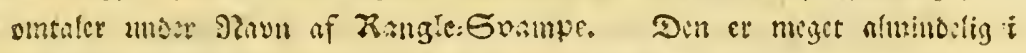

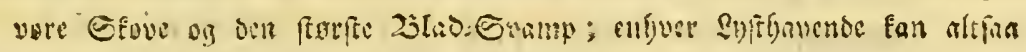

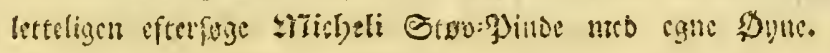

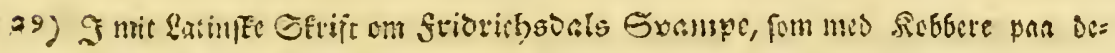

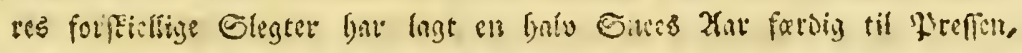

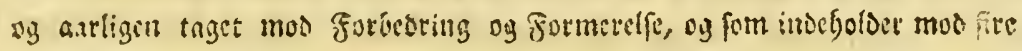

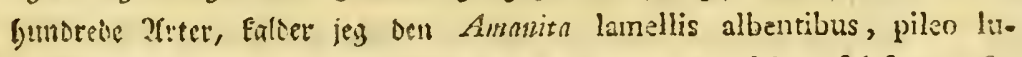
refcente, ftristo, villis pendulis; ftipite longo, cylindrico, fubfquamofo, 
wer fig i ttorbentlige Silumper, of fer Situten af Silabets ene

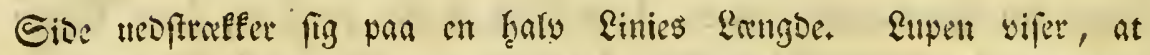

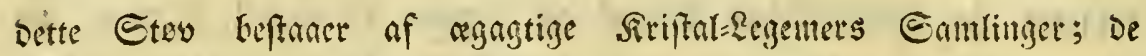

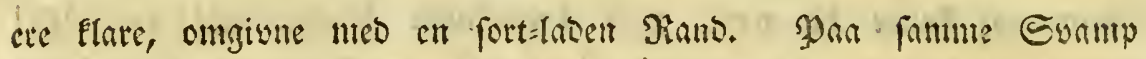
feer man og nogle fan lange Sranbe, font lobe pan Tuers over Brndenc. Man vil troe, at be ere Epillo af en Spinde, fyucr: for jeg og i mange Aar f̧ar anfeet bem; men, f̧vis man bringer Dem unoer Forfterrelfes= Siloffet, faa beftane difipe lange og tyffe

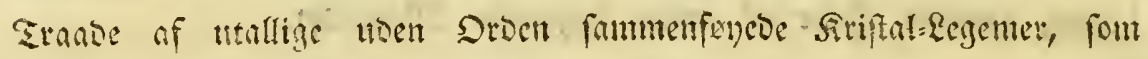

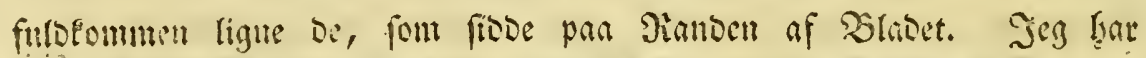
i 24 Simer betragtet Dem mber forfterelfen i naturligt og lunfer

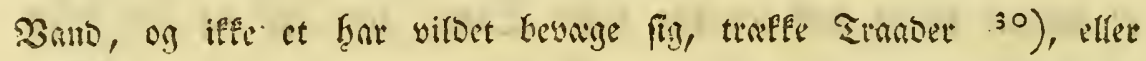
bngge nogen Eyamp. Dog maa man yogte fig, at iffe alt fee cir lloen fra henterende Bewregelie $i$ de paa Bandet furs

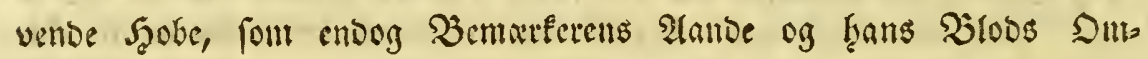
$\mathfrak{l a b}$

ammulato, bulbofo. Siboer wout Sinne bar ben iffe; bos Sarou von baller frace ocn Enum. ftirp. Helv. $237 \mathrm{I}$; og Teyninger af Den fecs gob Sterbcect theatr, fung. t. 7. f. A, i $\Lambda$ ct. Helvet, vol. IV. t. 5. f. I.

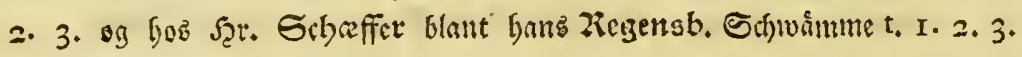

so) Mlan fan iffe vere langlom nof i nt beobmme viffe Syner under Mifrs:

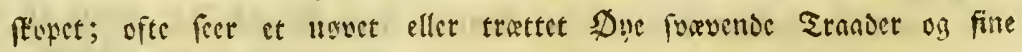
Sibe, fom iffe ere tif uben for famme; iffe fieloen foreftilfer bet pas

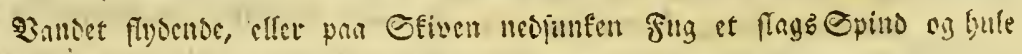

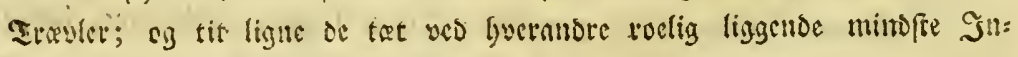

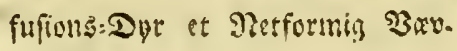




\section{9}

leb four fornarfage for frivillig, $\mathrm{eg}$ formb forfifere fin, at ingen af

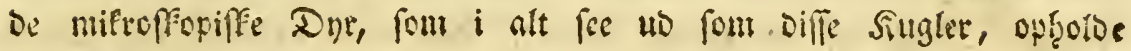
fig $i$ 2andet.

Email = Sompen $\left.{ }^{3 x}\right)$, fivis Şattes Sucrfabe er overbraget

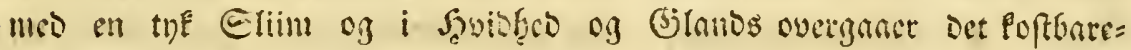

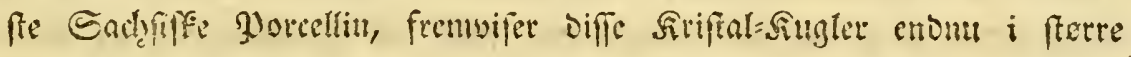

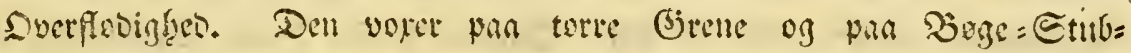
ber, oj gav mig engany et Evn, f̧uis Tifblivelfe jeg instil Dente

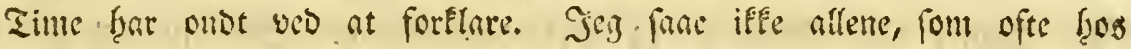

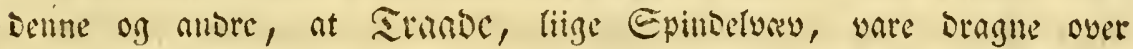
Dens blabe, men at biffe Srande entog moftrafte fig then for Evampen over bent bosftaachoe Bage= Stumme, Dens funa Brene og grenne Bitade $i$ mange 3 entinger, og at nogle of Dem banthte

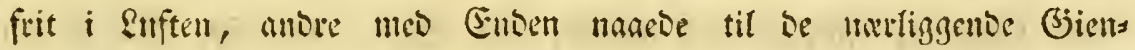
ftande. Singen Eltnoe anfec Det for anbet eno Epinselvary, og jeg fravoe $i$ ben Tanke, ligefom forţen, iffe panantet bet, fruis iffe ben ḩos

3x) STant Frioerichgoals Gyampe foocr den: Anunita lamellis niveis; pi. leo candidisfimo, vifcido; caule annulato. Dent Ean yere Fungus va. gus alter, Batar, Arimin. 3T. t. 8. C. D. E. Efter Seffrivel= for er ben Şr. yon Rimmes Agaricus 1988 i bant Fl. Syec. Fom fan tilligemes 17 aliore 20 las: Svampe, efter at bave beficnotgietot bem $i$ ttende Efrifter, uemlig Ganz Flora Lapp. Janz FI. Svec. 1745. og Fi. Sv. 1755. Jart, uoen at angive 2(arfagetr, $i$ finte the Ulogaver af. fpecies plantarum og fyftema natura fat uto of Efafuningernes Sirgifeer. 


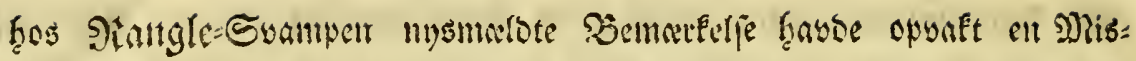
tanke. Nogle of be gremite blade, fom vare narmeft ved Goam=

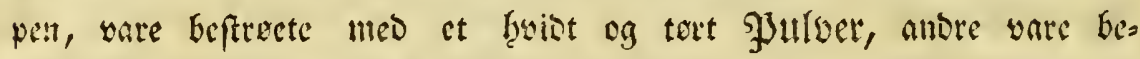

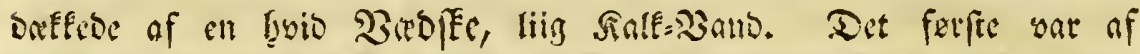
ben fyise Etev, fom yar falsen fra Evampens Blabe, og set

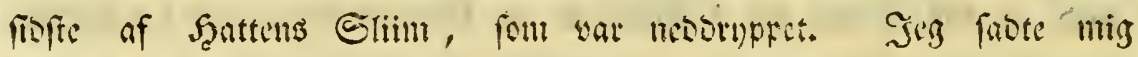
para mine Sink for at betengte bet mes all forfigtigfeco, og funte

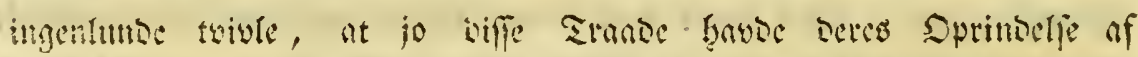

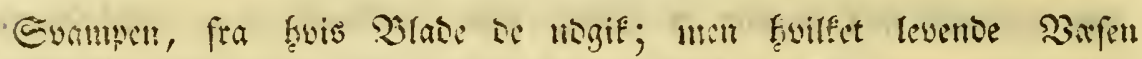
farr baunct biffe Errabe, og fort sem fra ct Cted til bet anbet

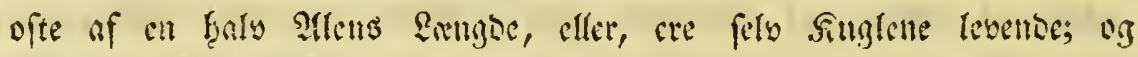

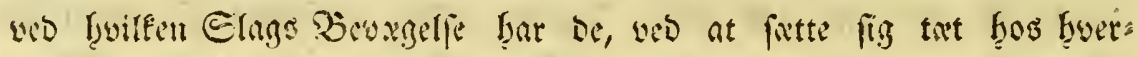
anber, Eumbet frembringe siffe Franbe? Jig fortso siffe og anbere Sparsman, og ileoe friem, i bet Scanh mu, huis nugenfinse, at fee

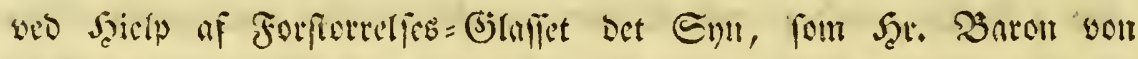

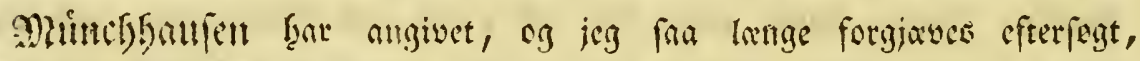

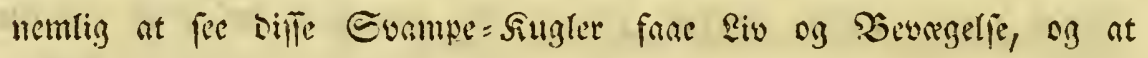

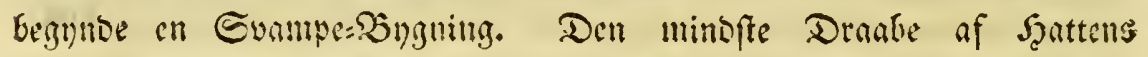

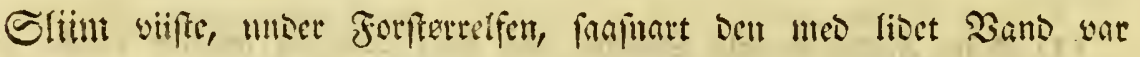

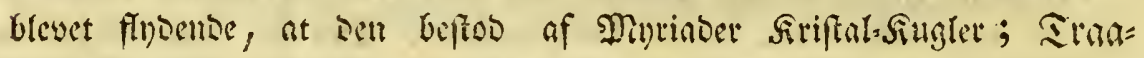

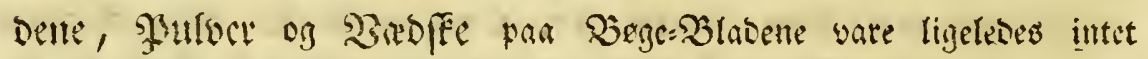

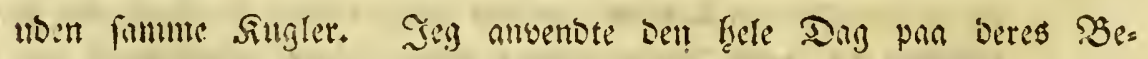
tragtning; ingen vilde bevarge fig; Det forftaner fig, at be oven $i$

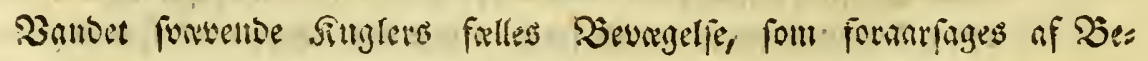


merfercus Aanden, Futz: Elag, Borbets Seorering, iffe maa ag= tes. Seg lagoe bem $i$ llutet og fold $3 a n d$, fom var foyst, men omfonff. Endeligen bemarfede jeg $i$.nogle fiugler en fanofrordig og. frievillig Sevargelfe of en gandfe egell $\mathfrak{A}$ trt. Tuende overmaat be fimaa og flare Sittgler fontes at vere foblebe til fiveranore, og

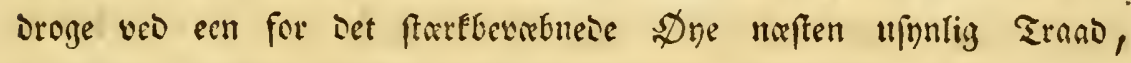
ber yar tre Bjange far lang foum en Siriftal = Sittgle, en fandant Sitgle efter fig. De foentmiebe omiring med den i alle Bendin= ger. Det var mig, noget farfomt; Seftere vare $i$ oit minofte fite

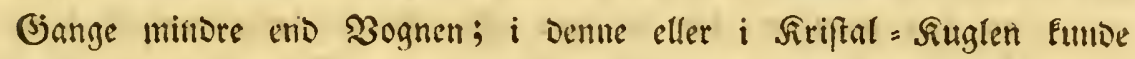
jeg ille fpore nogen egen Brevegelfe; iffun twende of biffe havoe

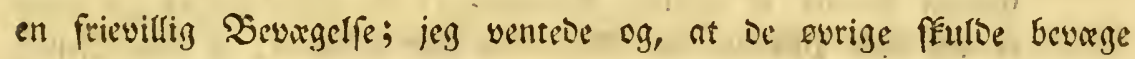
fig, men biffe tminto bleve ulevergelige, og $i$ bercs. Nioe. Nange fma Sungler, liig Dem, fom vare Forfan, gié. ma omfring uben 3ogne; og en forre Sort af Efobning fom ell Einfe los fig mut til Enne. Dette face man, ba 3anbet naften war utodunfet; faa junt ieg gav Dem friffet Band, Eunbe jeg iffe mere marte no= gen Severgelfe Hos Sriftal=Singlerne; Forfpans:Suglerne Detimoo va=

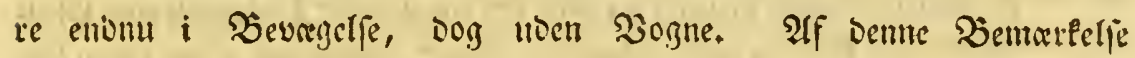

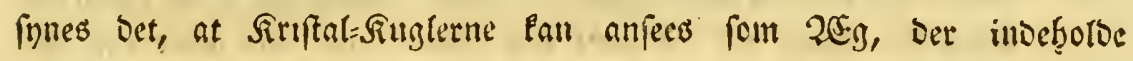
enom minore Siugler, fom blive levente, frette 2erggene i Bscure gelfe, bryoe us af ocm, og. unbertisen paa nogen Tio flecbe beres 2Egge: Seal efter fig. 
Dette er ien farfte Song jez efter manige forfeg og

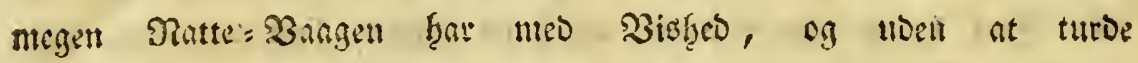
gise min flere Inowendinger it agt taget nogen fricuillig Sevargelfe:: $i$

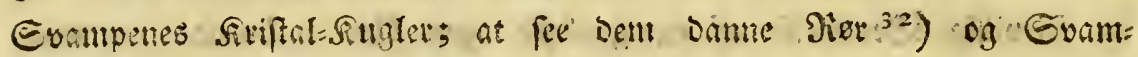

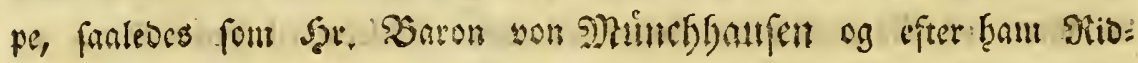

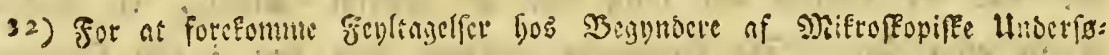
gelfer maac ieg of mange Bomerefler, foum jeg, for at fee diffe Ey=

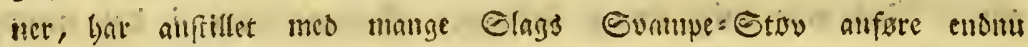

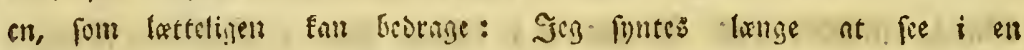

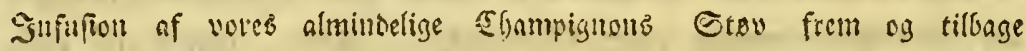

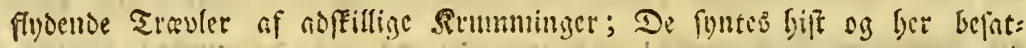

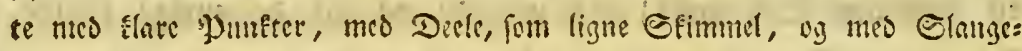

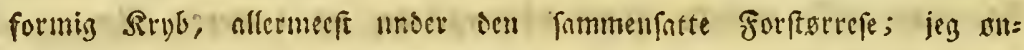

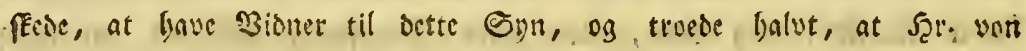

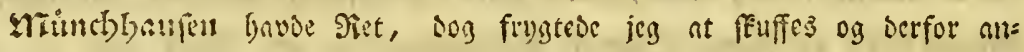

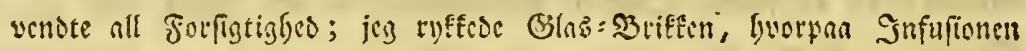

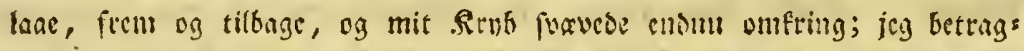

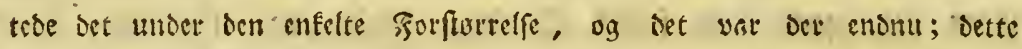

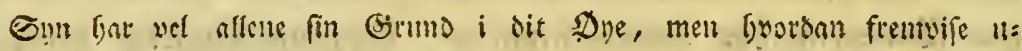

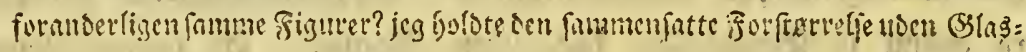

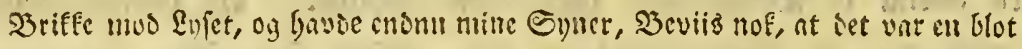

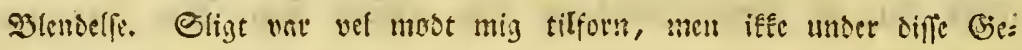
frafter. Dog torde jeg iffe troe, at Şt. yon 2rT. Gavde Gedraget fig.

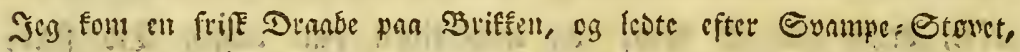

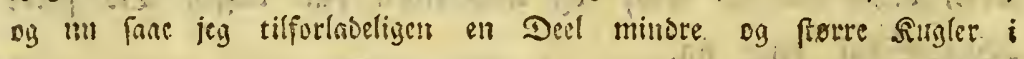

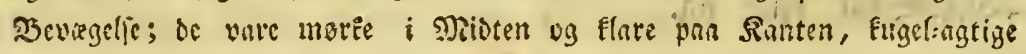

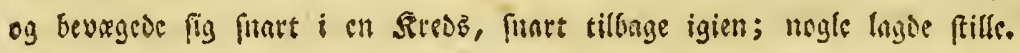

StD: 


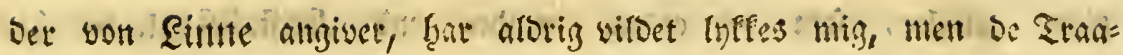

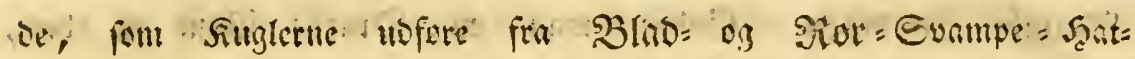

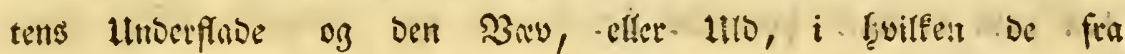
Rol=Evampene ubfarente Sinuler og Singler forcene fig par beres

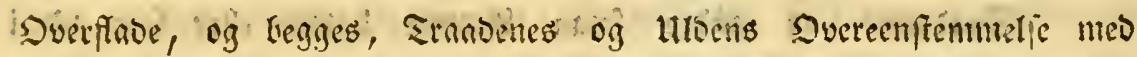
De Tranbe og ben uroagtige Efimnel 's3.), fom fintes yed fooen af Evampene, og cr biffes forfte Beguntielfe, labe intgen Suivl, at

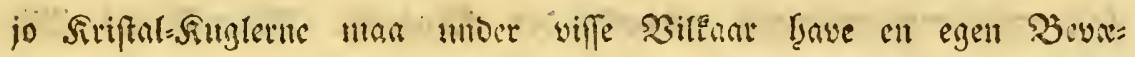

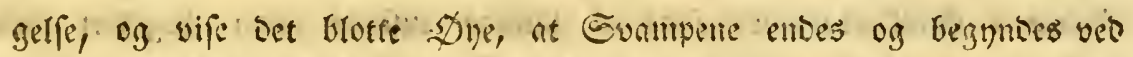

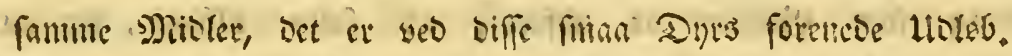

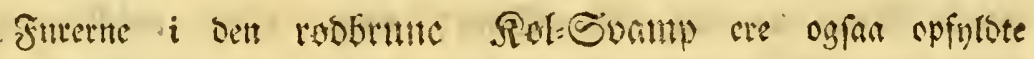

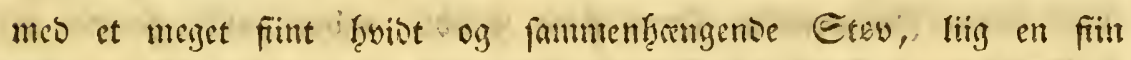

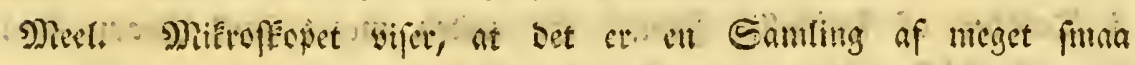
.

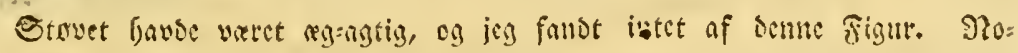

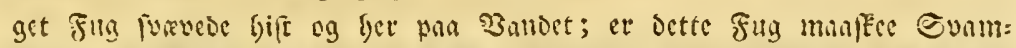

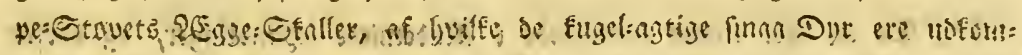

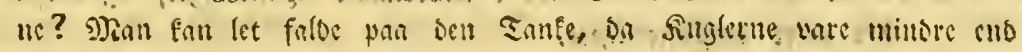
oe forger feete Brem af Sunmpe:@tavet.

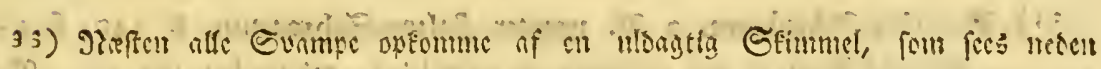

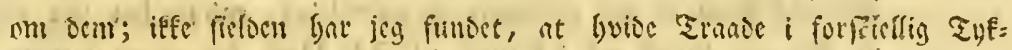

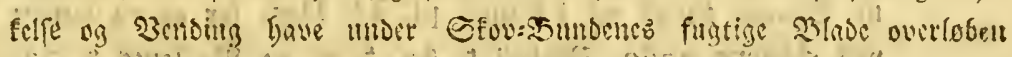

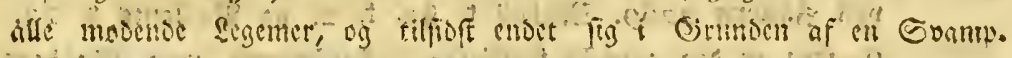

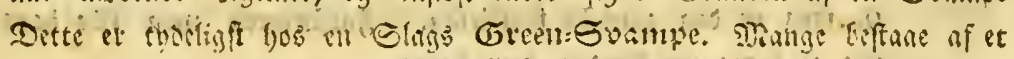

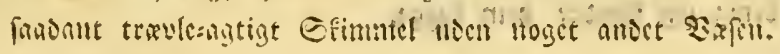




\section{4}

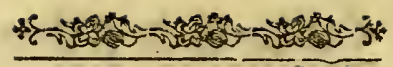

Flare Siugler, fom ere enonu minore eno friftal=-Sitglerue tyos Sangle= Srampent. Binoer: man, en Draabe Band til Dem, fan man betragte ḩuer for fig.

Saabanne og anbre: fimaa Itomer bampe eller fare 110 i Etrosmal af Fold: Svampene, og of Den rode, og af ben forte

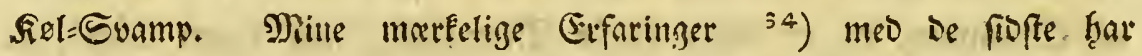

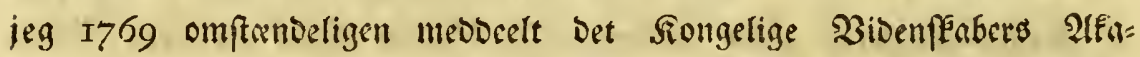
Demie i Stoffrolm; jeg frae of famme tufinde fmaa egemer fom Pinde og fom Ginifter at ubfare, at falbe tilfage, og at famle fin til en fiin inee=hyid Lllo. Seg opfangede Dem nogle. Sange paa en Gilas=Sfive; nogle bleve enfelte, og lig= neve under Forforrelfen fma frive, Soinde, andre havbe forentet fin i Sraaber of forffiellig Fart.... En anden meget fmue Evamp, fom Sar. Schåffer 35; alleme tilforn haar fulloet, forefom mig i Spu= rep|Eitl

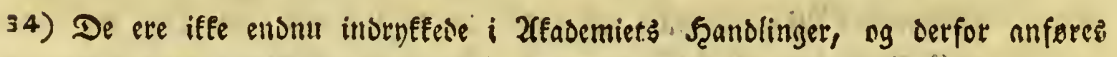
Jer intet omftenbeligere.

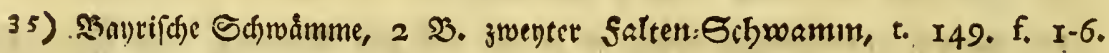

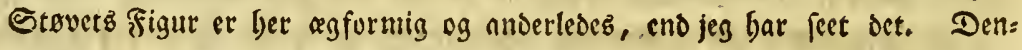
ne Sunmp anjeez vel $i$ Enum. pl. Fl. Dan, for en og fanme med Şr. son. Jallers 2269. NRicheli t. 82. f. 2. og 33nillants t. 13. f. 7.8 . 9. men er bog gandfee forfteillig. Forfatteren maa iffe bave feet no: gen af bem; jeg tiender bern begge. 


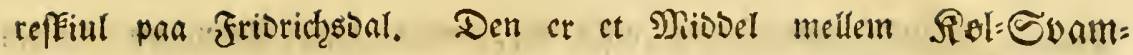
pent og Folbe=Soampen; Den thofafer fmaa Travler, rigefoum Den robe Rol=Swamip; jeg thar frap beforget ben malet for Flora Danica tilligemed de ubfarende Trevuler forfforrede. Befmnderligt not er bet, at biffe Itomer ere af forfficllig Efabning bos Evampe= 2trter af en Sregt, og thos en anoen Slegt af en og famme; Den rob=brume Sol=Syamip gav flare Fugler, Den robe brnelige Trav: ler, og sent forte frive Pinde; men alle B̉nd: Evampe=Arter give,

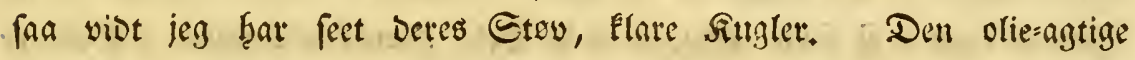
Broffe, f̧vormed en Deel Evampe overtraffes, er fros nogle intet altoet ent en forening af ovenmelote Fillgler, og fyoor mange fine

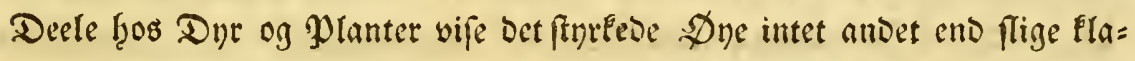

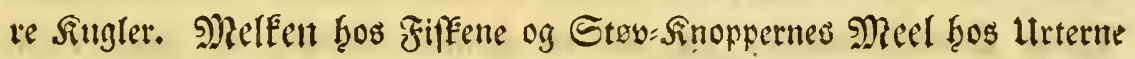
beftane af faabanne fig bevagende fugleagtige Regemer, og vi veco, at Sroen. has Dyrene $i$ Almindelighted er opfylot med. flige af forffiellig Bieftalt.

Man foreftille, fig mi i fulo Modenţed en Grund:Tegning, cn Epire, en Emurno, eller huad man vil Ealte bet, fom anfalbes af Millioner of biffe fiman engelagtige, og ved ell gelinde Barme i Bevregelfe bragte Eegemer, ber intrange fig $i$ Detl, og af Nerrings:@aften Dagligen underftettes meo Mintiaber Piefrnter; og man vil uben 3anffectighede begribe, at oenne Epire eller (5m= bryo vil $i$ fort itio blive finlig, vore og opnaae fin. Futofom= 


\section{6}

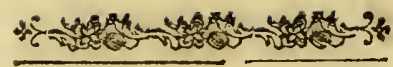

menhed. Fulbenmmentreben vifer fig, naar siffe Mrbendere famtes

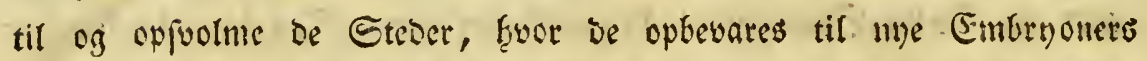
Hlovifling. Saaleves ende og beginte de alle Dyrs og Mlanters ßept og Mobenfeco.

Dente Iolings Theorie, engang faftabt, wil blive frugtbat

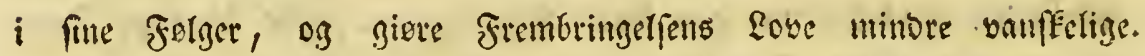
Efabningernes. Mamtigoe og $\mathfrak{B r r t}$ ma tittage $i$ fortyold til Forraaben of biffe fina Dur, fon de Dele, fivoraf be befrane. Fyuor er Deres Snerflebigfed frere end i 3and=Sirefter, Eumper, פiofer,

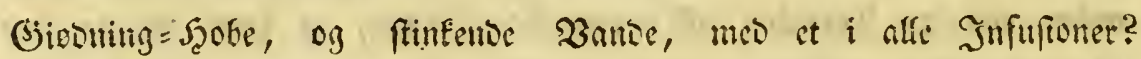

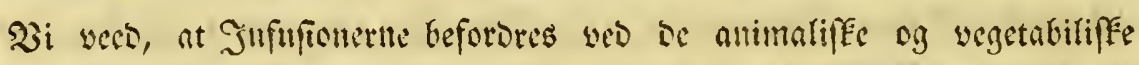
Deles Eamberffireclfe, og yed en gelinbe Barme, og ingen er th

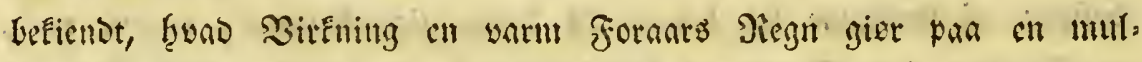
Det og uel forarbeidet Sorb, eller Biebningen pa en mager og it=

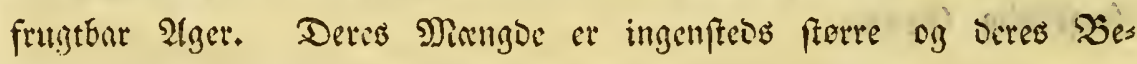

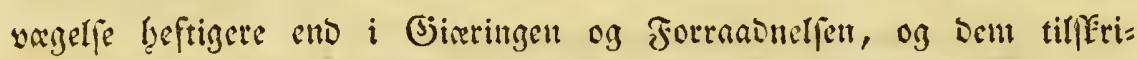
wer jeg sen. ultidelige Etank, fon 3rofferne Da give fra fig. Dog en fa vigtig Materie ber fremlegges mere omfternderigen, og for urrwerchise Tio var bet allene mit Forfat at beffrive en Earves

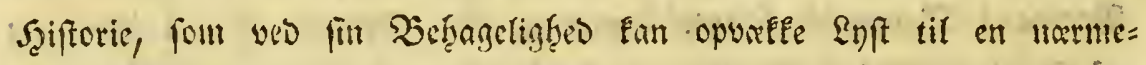
tr Befiendeffar mes yore egne Entes naturlige frembringelfer; og Sette tilfaber mig neppe mete eno et Hoeaft af mine Socer our Tstinget. 


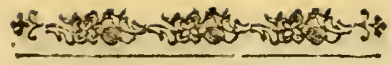

\section{7}

Bores Earve bliver 3interen over $i$. fill Wuppe og frembrys

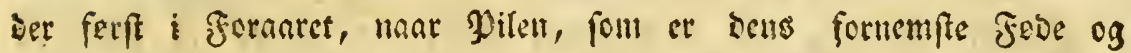

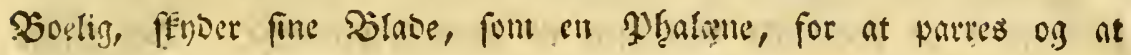

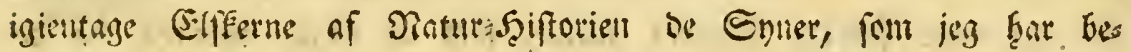

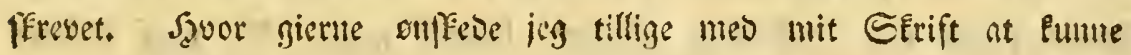
overgive min Eafere Exemplarer of Diffe Earver, og $i$ Jolge of bes

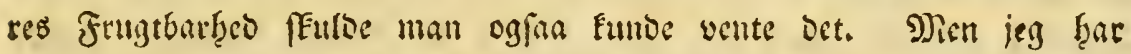

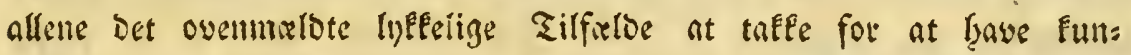
Det medocle Dens feiftorie; $i$ fem $\mathfrak{A}$ ar uogiorbe ben ell Deel of $\mathbb{E}$.

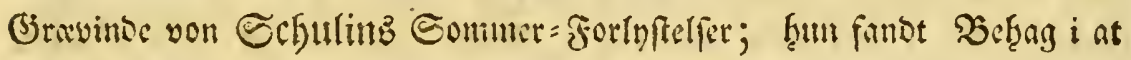
nofluke og plene ien, og at tilfee Dens Daglige Tiluept og for:

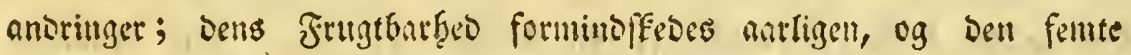

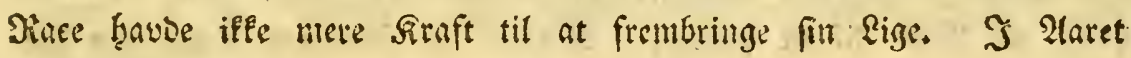
I765 fremfoum of Galvtreofinostive, fom ind fpundue havose i beres Puppe 3interen oyer faaet $i$ furvaring paa adfeillige Eteocr, iffun fire, De evrige vare $i$ Wupperne fortartebe og til Emul blevite. Waa

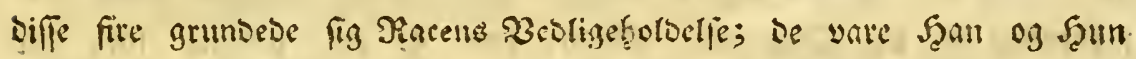

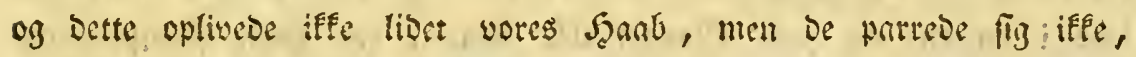

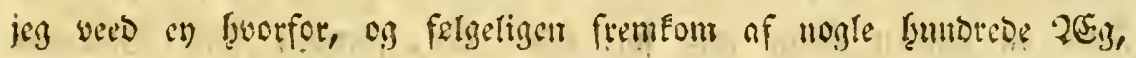

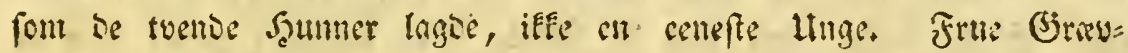
illoen ubfatte Prifer for bem of hembes şumb=suls, font fanbt bet=

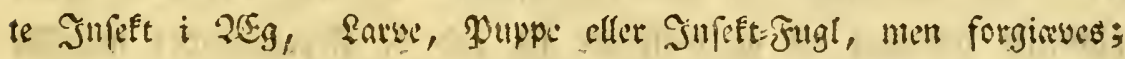

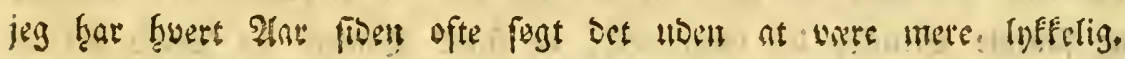




\section{8}

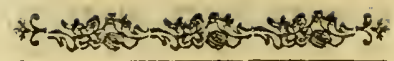

Saa mange Pluppers $\mathfrak{T} a b$, og meo bem Scanbets forliis om tilfoumetroe Earver, gik mig nojet nar; jey finutes at finde en Sinoring $i$ at eftertinke 2 arfangen til Deres Forbervelfe. Det funbe iffe vel yere forrige Binters Siulbe, fom of nogle meentes, $D a$ be

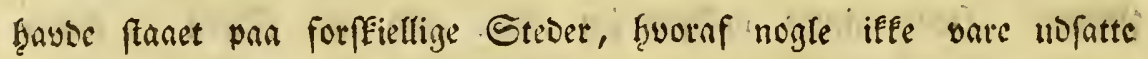

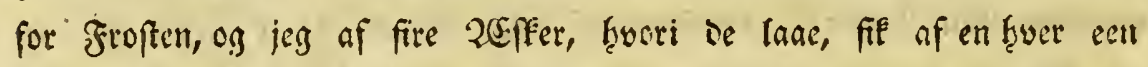
levende; Destloen vare alle de, fom $i$ de forrige Aatinger havode insfputroct fig, ubfonume i Deres Fugle- Siefralt, woen at en enefte

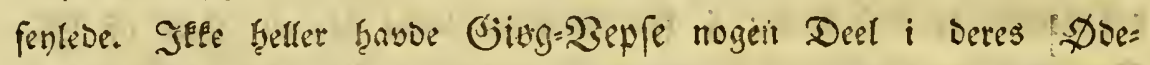
leggelfe, thit $i$ fen 2 tar var ingen blewen angreben of Diffe Biefter. Enarere fintoe man giette paa en indyortes Starfag, fom frar fin= Dret beres fiofte forwanding, og betaget be fane, fom opnaaede

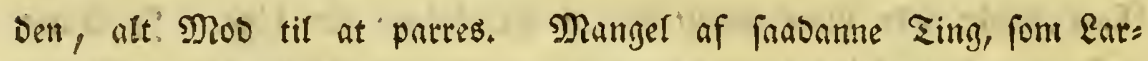
verne Gave-i Deres Friebico, og fom fattes sem $i$ et Saummer, et maafere sen fanbe. Deres Forterrelje til et brunt Emul giver tils

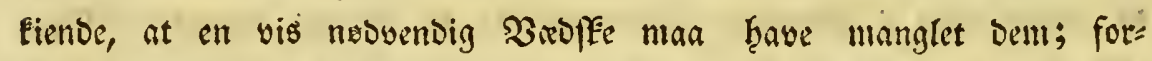
modentlig er bet. Dug eller Jiegn, fom be ere ublatte for, ber leve $i$ Enften, og fivis Mangel iffe freak $i$ ben forfte froutplantelfe forer en faaban Folge med fig, fioen Der, foruben Pile = Blabenes egen Saft, endog uben paa be Blabe, fon bagligen gaves bem til Fobe, ofte vebhannger ent Deel Fungtigheco. Dg var ben fiofte Fort: plantelfe falfigete til at intopinde fin ento be Foregaaente; maafeet

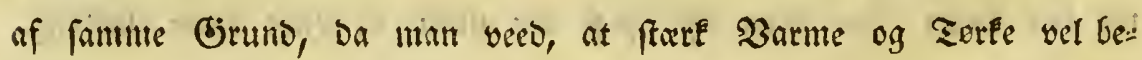

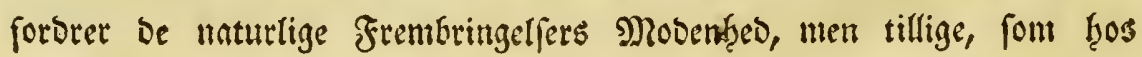




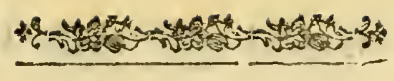

\section{9}

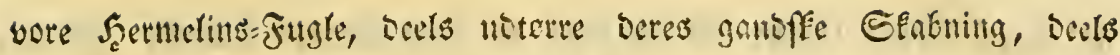

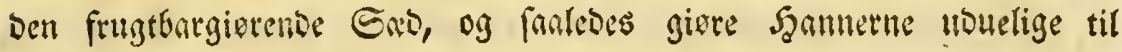
Parritig.

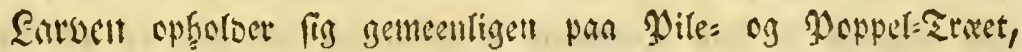
mere fretoen paa Sitke og Ege= Traect. De gamle falbe sen Vinula, formoientlig of Den viin=reoe farve, fom ben, fauteoes foul bioes Dart bar afmalet ben paa Din 65 Tavele, antager fort for fin Stros:

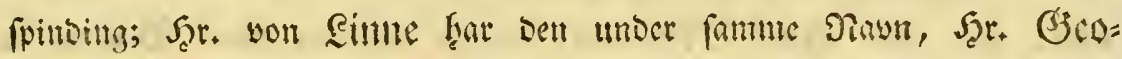

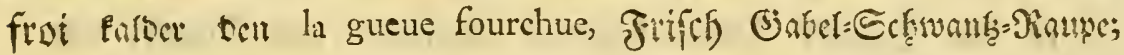

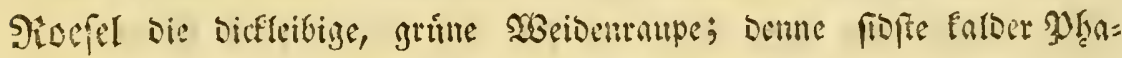

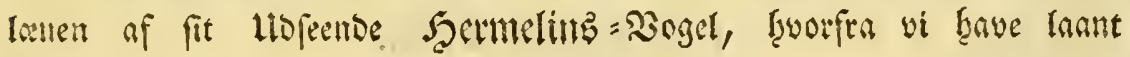
Bavinet.

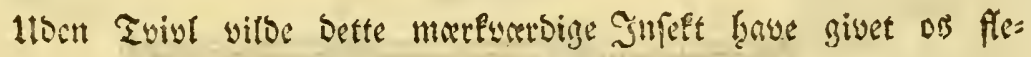
re kefinnerlige Natur=Enner, fyuis jeg $i$ se Rar, jeg havbe Sver=

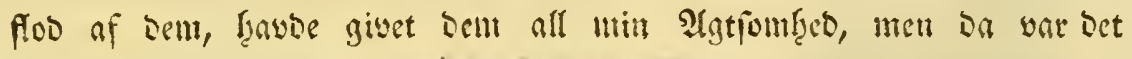

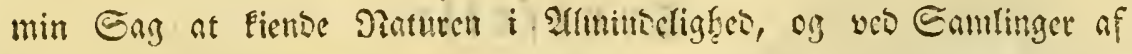
Det, fom Danmemare frar tiffalles cller fertoetes frem for anter

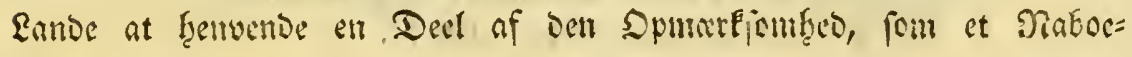

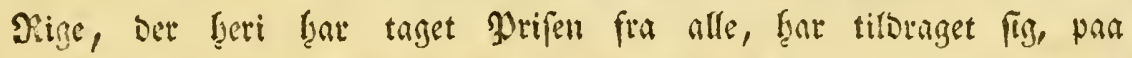

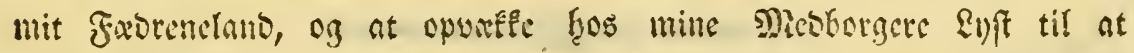
giere Hltierfegelfer, uoen for ben $T$ io at befatte mig mod cufelte Deles Şiftorie. Mianfee en Misfornturle ouer at fee mig, man= 


\section{0}

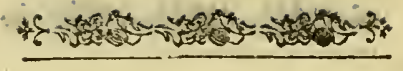

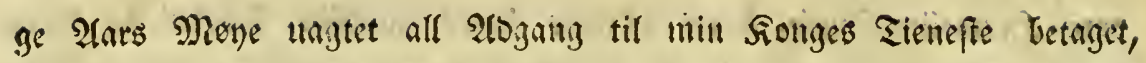

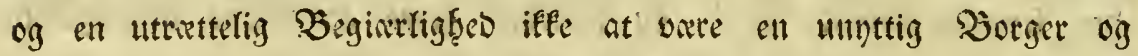
fravoe fin Deel heri; og Da ter jeg vente, at Sitendere sille tils fane mig, at, fywis jeg of De Midoler, foun frobe i min frie Billie, hant novalgt be para Formaile frugtbarefte, Det Deg cy ere bleune De mageligfte. Sfulbe nerverente Efrift, Det altoet, fout jeg fertivet i mit Mobers Manl, iffe blive mine Ranbsimento aldeles ligeguloigt, wil bet yare mig en folclig fornobelfe, heller at funne avergive Dem, fan viot Şans Majefterts Tienefte tillader, mine Bemevelfer

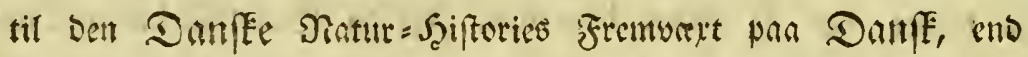
imos min gooe 3 illic at fee mig nebjaget at pertive $i$ et fremmet Eptog.

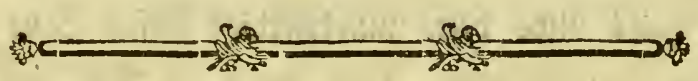

\section{$\mathfrak{T} \mathfrak{i} \mathfrak{l}$ Efeil:}

Ois. 7.23 Sinic; Bcountelfe, 1. vedrstelfe.

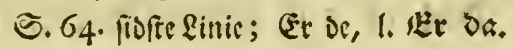

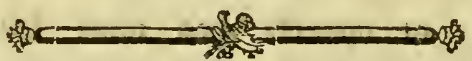


I. Taule.
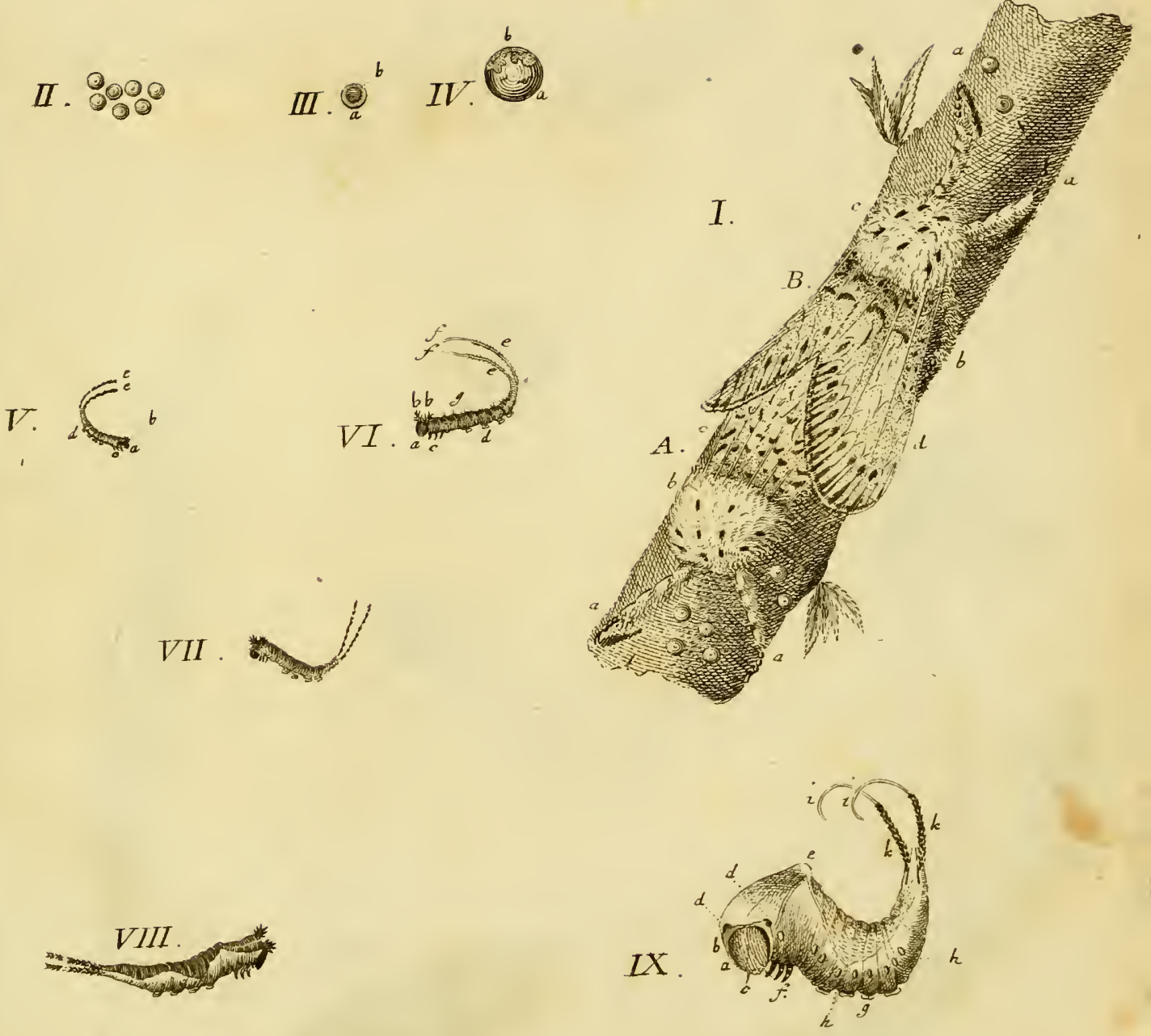

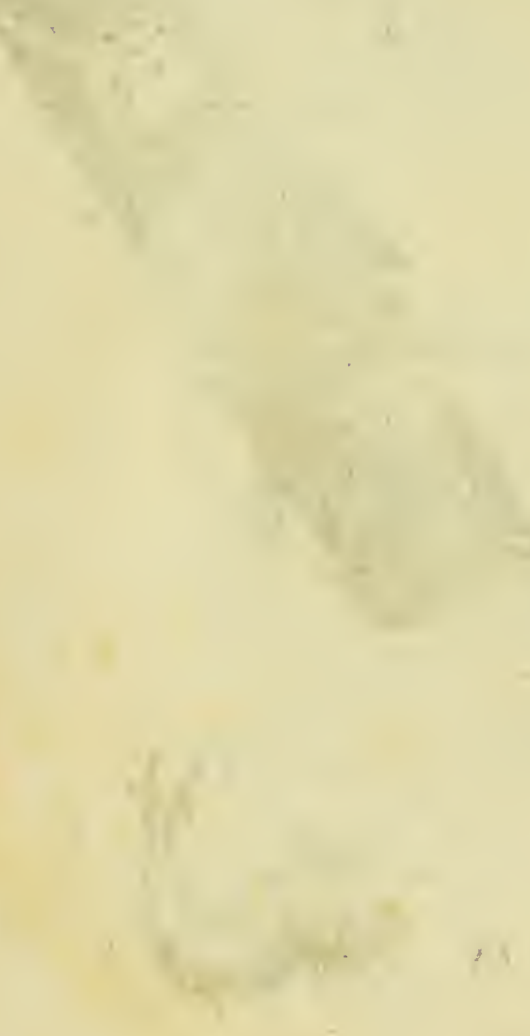


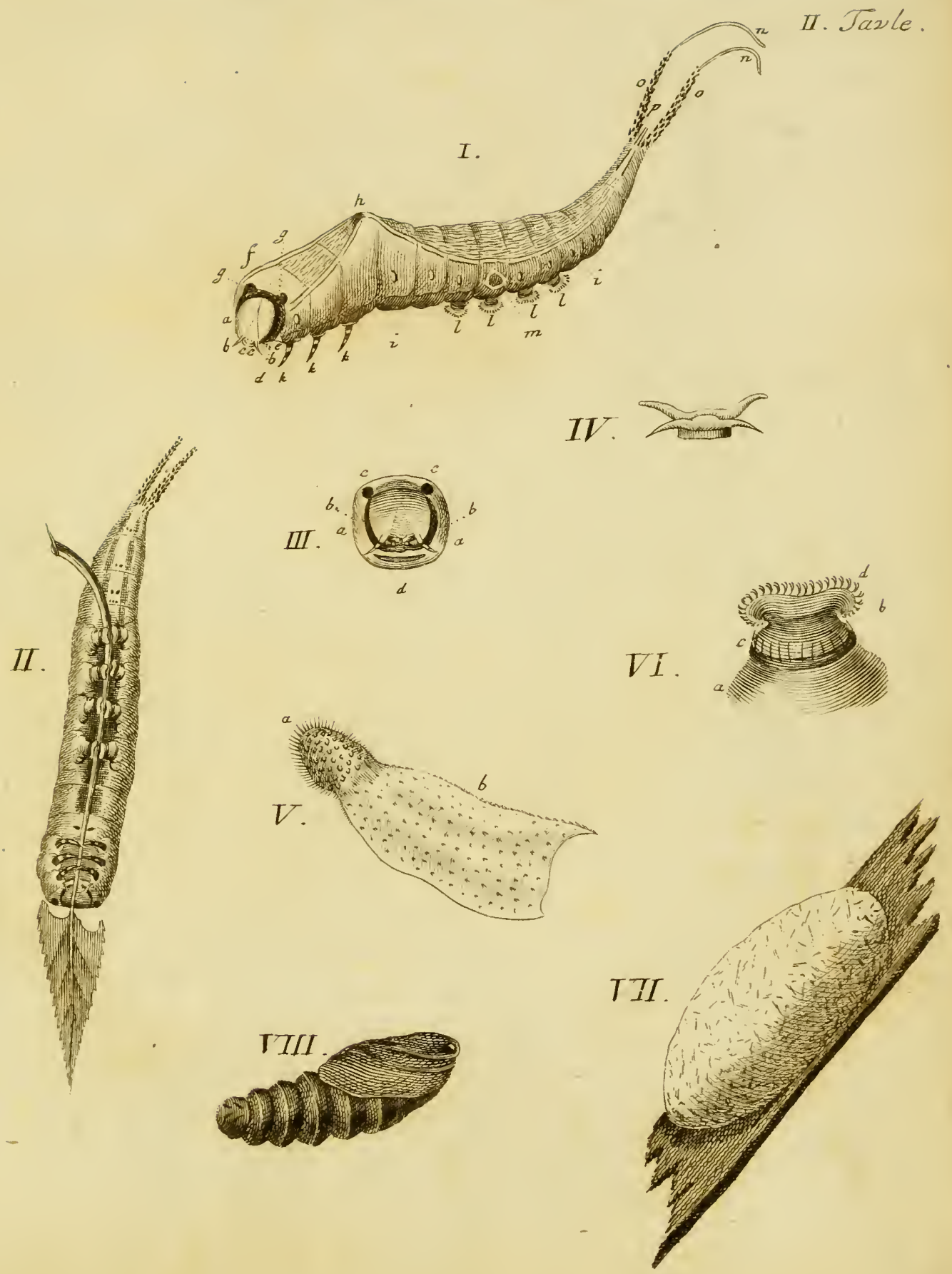





$$
400
$$




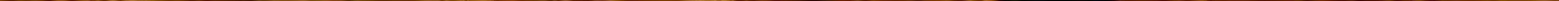

